\title{
Analysis of Reinforcement Designs for Specials in Steel Pipelines
}

\author{
by
}

Reginald Murray Barry

Thesis presented in partial fulfilment of the requirements for the degree of Master of Science in Mechanical Engineering in the Faculty of Engineering at Stellenbosch University

Supervisor: Prof. G. Venter 


\section{Declaration}

By submitting this thesis electronically, I declare that the entirety of the work contained therein is my own, original work, that I am the sole author thereof (save to the extent explicitly otherwise stated), that reproduction and publication thereof by Stellenbosch University will not infringe any third party rights and that I have not previously in its entirety or in part submitted it for obtaining any qualification.

Date: March 2017.

Copyright (C) 2017 Stellenbosch University All rights reserved. 


\title{
Abstract
}

\author{
Analysis of Reinforcement Designs for Specials in Steel \\ Pipelines \\ RM Barry \\ Department of Mechanical and Mechatronic Engineering, \\ University of Stellenbosch, \\ Private Bag X1, Matieland 7602, South Africa. \\ Thesis: MScEng (Mech) \\ March 2017
}

This thesis relates to the analysis of reinforced steel pipe specials (tees and lateral tees) for bulk water pipelines. Pipe specials provide a means of dividing and/or uniting flows. Finite element (FE) analyses are performed to evaluate the, experimentally obtained, American Water Works Association's (AWWA) M11 design model that is commonly used.

Modelling considerations are discussed with emphasis on stress-strain behaviour, boundary conditions and geometry. The material non-linear behaviour of steel is described, with emphasis on the selection of suitable numerical analysis tools. The purpose of reinforcements for large diameter steel specials are explained and the types of reinforcements are described in detail. Emphasis is placed on the development of a large number of simulated 45 degree lateral tees. Possible model variations and alternative types of reinforcements are briefly looked at.

The development of adjustment parameters for M11 designed reinforcements are discussed, with consideration to the mechanical properties of modern steels. The results of physical testing were used to validate the numerical models developed in this study. The deformed shape of the pressurised test piece used in the validation process corresponds to the numerical model and strain measurements correlate well with simulated strains. 


\title{
Uittreksel
}

\section{Ontleding van Versterking Ontwerpe vir Koppelstukke in Staal Pyplyne}

\author{
("Analysis of Reinforcement Designs for Specials in Steel Pipelines") \\ RM Barry \\ Departement Meganiese en Megatroniese Ingenieurswese, \\ Universiteit van Stellenbosch, \\ Privaatsak X1, Matieland 7602, Suid-Afrika. \\ Tesis: MScIng (Meg) \\ Maart 2017
}

Hierdie tesis het betrekking tot die ontleding van versterkte staalpypkoppelstukke (T-stukke en laterale T-stukke) vir grootmaat water pyplyne. Pyp koppelstukke maak dit moontlik om vloei in 'n pyplyn te skei en/of te verenig. Eindige element (EE) analises is uitgevoer om die eksperimenteel ontwikkelde M11 ontwerpsmodel van die American Water Works Association (AWWA), wat algemeen gebruik word, te ontleed.

Modellerings aspekte is bespreek met klem op spanning-vervormings gedrag, grenstoestande en geometrie. Die materiaal nie-lineariteit van staal is bespreek, met klem op die seleksie van geskikte numeriese ontleding hulpmiddels. Die doel van versterkings vir groot diameter staalpyp versterkings word verduidelik en die verskillende soorte versterkings word in fyner besonderhede beskryf. Klem word gelê op die ontwikkeling van 'n groot aantal gemodelleerde 45 grade laterale T-stukke. Variasies op modelle en alternatiewe soorte versterkings word vlugtig bespreek.

Die ontwikkeling van aanpassings parameters vir M11 ontwerpe versterkings is bespreek met oorweging van die meganiese eienskappe van moderne staal. Die resultate van fisiese toetse word gebruik om die numeriese modelle wat in hierdie studie ontwikkel is te bekragtig. Die vervormde drukbelaste toetsstuk wat gebruik is vir die bekragtigingsproses vergelyk goed met die numeriese model en die gemete vervormings korreleer gunstig met die gesimuleerde vervormings. 


\section{Acknowledgements}

To our Heavenly Father for blessing me with a healthy mind and the will to continue learning.

I would like to express my sincere gratitude to the following individuals and organizations:

- My wife, Annette - this thesis would not have been possible without your encouragement, patience and support.

- Prof Gerhard Venter for the professional support and guidance he provided as a study leader

- My parents, Reenen and Briëtte Barry, for supporting and encouraging me.

- My colleague, Schalk van der Merwe, for his continuous support at work while studying on a part-time basis.

- My colleague, Stephan Kleynhans, for his encouragement and assistance.

- Jaco van Blerk, Jan Cloete and Kiewiet Viljoen for their assistance, generous donations and support in manufacturing of the test piece.

- Altair for FE software support and Esteq for assistance with data analysis equipment and software support. 


\section{Contents}

Declaration $\quad$ i

Abstract $\quad$ ii

Uittreksel iii

Acknowledgements

Contents $\quad$ v

List of Figures $\quad$ vii

List of Tables $\quad x$

1 Introduction $\quad 1$

1.1 Background to the Project . . . . . . . . . . . . . 1

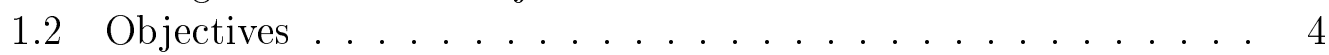

2 Literature Study $\mathbf{5}$

2.1 Mechanics of Steel Pipe Specials . . . . . . . . . . . . 5

2.2 Introduction to Stress Concentration Factors . . . . . . . . . . 6

2.3 Reinforcement of Specials for Steel Pipelines . . . . . . . . . . 11

2.4 AWWA M11 Design Model . . . . . . . . . . . . . . . 16

2.5 Introduction to FE Methods . . . . . . . . . . . . 27

3 Finite Element Analysis of Cylinder-Cylinder Intersections 29

3.1 Numerical Modelling . . . . . . . . . . . . . . . . . 29

3.2 Problem Classification . . . . . . . . . . . . . . 29

3.3 Mathematical Model . . . . . . . . . . . . . . . 31

3.4 Preliminary Analysis ... . . . . . . . . . . . . . . . . . 31

3.5 Finite Element Analysis . . . . . . . . . . . . . . . . 32

3.6 Modelling of Steel Specials . . . . . . . . . . . . . . . . . . . . . . . . . . . .

3.7 Numerical Results . . . . . . . . . . . . . . . . . . 48

3.8 Model Variations . . . . . . . . . . . . . . . 57

3.9 Alternative Reinforcements . . . . . . . . . . . . 62 
3.10 Modelling Conclusion . . . . . . . . . . . . . 67

4 Physical Testing $\quad 68$

4.1 General and Objectives . . . . . . . . . . . . . . 68

4.2 Test Piece Manufacturing . . . . . . . . . . . . . . 69

4.3 Experimental Setup . . . . . . . . . . . . . . . 70

4.4 Tensile Testing . . . . . . . . . . . . . . . . . 73

4.5 Experimental Procedure ........................... 75

4.6 Discussion of Experimental Results . . . . . . . . . . 76

5 Validation of the Numerical Model $\quad 81$

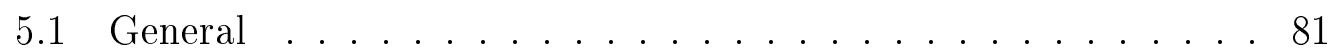

5.2 Visual Comparison . . . . . . . . . . . . . . . . . 81

5.3 Numerical Comparison . . . . . . . . . . . . . . . . . 83

5.4 Summary of Numerical Model Validation . . . . . . . . . . 88

6 Conclusions and Future Studies $\quad 90$

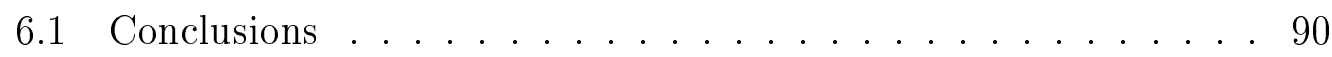

6.2 Future Studies. . . . . . . . . . . . . . . 91

$\begin{array}{ll}\text { List of References } & 93\end{array}$

$\begin{array}{ll}\text { Appendices } & 95\end{array}$

$\begin{array}{ll}\text { A Crotch Plate Nomograph } & 96\end{array}$

$\begin{array}{ll}\text { B Pressure vs Strain Curves } & 97\end{array}$ 


\section{List of Figures}

1.1 Typical plain ended radial (90 degree) and lateral tee. . . . . . . . 1

1.2 Two plate crotch plate manufactured in the early $1930 \mathrm{~s} \ldots . . .22$

1.3 Types of reinforcing plates for tees. . . . . . . . . . . . . 3

1.4 Typical lateral tee with two plate crotch plate . . . . . . . . . 4

2.1 Elliptical hole in uni-axially loaded infinite flat plate. . . . . . . . 5 5

2.2 Stress distribution in a plate resulting from a hole . . . . . . . . . 6

2.3 Typical barrel flat patterns for radial intersections. . . . . . . . 8

2.4 Typical barrel flat patterns for non-radial intersections. . . . . . . . 8

2.5 Elliptical shape of branch/barrel lateral tee section . . . . . . . . 9

2.6 Inside crotch corner of non-radial intersection. . . . . . . . . . . . . . 9

2.7 FE modelling of specials with shell elements. . . . . . . . . . . . 10

2.8 Typical tee with collar stiffener plate. . . . . . . . . . . . 12

2.9 Typical tee with wrapper stiffener plate. . . . . . . . . . . 12

2.10 Reinforcement methods presented by EN13480-3. . . . . . . . . 13

2.11 Typical crotch plate design in accordance with EN13480-3. . . . . . 14

2.12 Two and three plate tees tested with strain gauges . . . . . . . . . 15

2.13 Two plate crotch plate at Sishen Mine . . . . . . . . . . . . 15

2.14 Collar or wrapper reinforcement of special . . . . . . . . . . . 18

2.15 Cross-section of wrapper reinforced lateral tee . . . . . . . . . 19

2.16 Allowable outlet area. . . . . . . . . . . . . . . . . . 20

2.17 Wrapper plate cross-sectional area. . . . . . . . . . . . . . 20

2.18 Allowable excess area. . . . . . . . . . . . . . . 21

2.19 Typical single plate crotch plate . . . . . . . . . . . . 24

2.20 Layout and plan of lateral tee . . . . . . . . . . . . . . . . . 24

$2.21 \mathrm{~N}$-factor curve for crotch depths . . . . . . . . . . . . . . . . . . . . . . . . 25

2.22 Q-factor curve for reducing tees . . . . . . . . . . . . . . 26

2.23 Top depth selection chart . . . . . . . . . . . . . 27

3.1 Flanges on typical wrapper reinforced tee. . . . . . . . . . 35

3.2 RBE2 element connecting center node to outer nodes . . . . . . . . 36

3.3 Effect of different boundary conditions . . . . . . . . . . . . . 37

3.4 Typical Autodesk Inventor stress analysis. . . . . . . . . . . . . . . . 38

3.5 Surface losses due to midsurfacing. . . . . . . . . . . . . . . 40 
3.6 Shell model of wrapper reinforced tee. . . . . . . . . . . . . . . 41

3.7 Barrel, branch and reinforcing shell surfaces not intersected. . . . . 41

3.8 Surfaces intersected and excess internal surfaces removed. . . . . . . 42

3.9 Midsurface meshed with offset elements. . . . . . . . . . . . . . . 42

3.10 Mesh refinement on collar reinforced tee. . . . . . . . . . . . 43

3.11 Convergence of numerical results . . . . . . . . . . . . . . . . . . . . . . 43

3.12 Mesh refinement on crotch plate reinforced special . . . . . . . . . 44

3.13 Mesh refinement on equal diameter wrapper reinforced lateral tee. . 45

3.14 FE approximation of surface interactions. . . . . . . . . . 45

3.15 Element overlap at branch, barrel and reinforcement intersection . . 46

3.16 Welding on reinforced tees . . . . . . . . . . . . . . 4 46

3.17 Intersection of reinforcing plate with barrel. . . . . . . . . . . . . . 4 47

3.18 Contact surface discretization . . . . . . . . . . . . . . . . . 48

3.19 Yielding and deformation of test piece . . . . . . . . . . . . 49

3.20 Comparison between wrapper and collar reinforcements . . . . . . 54

3.21 Effect of yield strength on crotch plate reinforcement . . . . . . . 55

3.22 Adjust pressure crotch plate design . . . . . . . . . . . . 56

3.23 Boundary condition effects on adjusted thickness crotch plates . . . 57

3.24 Curvature of crotch plates for unequal tees. . . . . . . . . . . 58

3.25 Impact of intersection angle on stresses . . . . . . . . . . . . . 59

3.26 Different wrapper shapes on equal diameter specials . . . . . . . . 59

3.27 Integral vs. pad reinforcing. . . . . . . . . . . . . . . . 60

3.28 Difference in crotch plate base and wye exit angles. . . . . . . . 61

3.29 Impact of crotch plate curving on stresses . . . . . . . . . . . . 61

3.30 Crotch plate reinforced tee restrained on all three open ends. . . . . 62

3.31 DN600/DN600 test piece with internal reinforcing plate . . . . . . . 64

3.32 Internal reinforcing plate moved to centerline of barrel. . . . . . . . 64

3.33 Crotch plate reinforcement in accordance with EN13480-3. . . . . . 65

3.34 Simulated model of EN13480-3 crotch plate reinforced special. . . . 65

3.35 SANS 719 related gusset reinforced tee . . . . . . . . . . 66

3.36 Gusset reinforced tee with a failure load of $4.66 \mathrm{MPa}$. . . . . . . . 67

3.37 Simualted stress for crotch plate reinforced tee . . . . . . . . . 67

4.1 Welded test piece prior to addition of reinforcing plate. . . . . . . . 69

4.2 Wrapper welded to test piece. . . . . . . . . . . . 70

4.3 Test piece. . . . . . . . . . . . . . . . . . . . . . . . . . . . . . . . . .

4.4 Sealing of internal strain gauge cables. . . . . . . . . . . . 71

4.5 Internal strain gauges with waterproof sealing. . . . . . . . . . 71

4.6 Strain gauge locations. . . . . . . . . . . . . . . 72

4.7 Test piece with strain gauges and cables. . . . . . . . . . . 73

4.8 Typical tensile test setup. . . . . . . . . . . . . . . 74

4.9 Stress-strain curve of test piece material. . . . . . . . . . . . . 75

4.10 Convergence of Poisson's ratio. . . . . . . . . . . . . 75

4.11 Typical unloading/reloading curve with reloading elastic strain. . . 77 
4.12 Typical pressure and strain recordings. . . . . . . . . . . . . 77

4.13 Pressure vs. strain plot. . . . . . . . . . . . . . 78

4.14 Normalised pressure and strain curves. . . . . . . . . . . . . . 78

4.15 Strain gauges "A" (red) and "E" (blue). . . . . . . . . . . . . 79

4.16 Strain gauges "D" (red) and "L" (blue). . . . . . . . . . . . . . . . . 80

4.17 Deformation of test piece acute corner. . . . . . . . . . . 80

5.1 Elastic perfectly plastic behaviour . . . . . . . . . . . 82

5.2 Small crack in acute corner. . . . . . . . . . . . . . . . 82

5.3 Typical failure of 90 degree special . . . . . . . . . . . . . . 83

5.4 Simulated deformation in acute corner. . . . . . . . . . . . . . 83

5.5 Approximate strain gauge locations. . . . . . . . . . . . . 84

5.6 Branch with negative strains . . . . . . . . . . . . . 87

5.7 Barrel and wrapper with negative strains . . . . . . . . 88

A.1 Nomograph for selecting plate depths . . . . . . . . . . 96

B.1 Pressure vs strain - strain gauge A. . . . . . . . . . . . . . 97

B.2 Pressure vs strain - strain gauge C . . . . . . . . . . . . 97

B.3 Pressure vs strain - strain gauge D. . . . . . . . . . . 98

B.4 Pressure vs strain - strain gauge F. . . . . . . . . . . . . . . . . . . . . . 98

B.5 Pressure vs strain - strain gauge K. . . . . . . . . . . . . . . . 98

B.6 Pressure vs strain - strain gauge L. . . . . . . . . . . . . . . . . 99

B.7 Pressure vs strain - strain gauge M. . . . . . . . . . . . . . . 99

B.8 Pressure vs strain - strain gauge N. . . . . . . . . . . . . . 99

B.9 Pressure vs strain - strain gauge O. . . . . . . . . . . 100

B.10 Pressure vs strain - strain gauge P. . . . . . . . . . . . . . 100

B.11 Pressure vs strain - strain gauge S. . . . . . . . . . . . . . . . 100

B.12 Pressure vs strain - strain gauge T. . . . . . . . . . . . . . . . . . . . . . . . . . . .

B.13 Pressure vs strain - strain gauge W. . . . . . . . . . . . . . . . . . . . . . . . . . . .

B.14 Pressure vs strain - strain gauge X. . . . . . . . . . . . . . 101 


\section{List of Tables}

2.1 Effect of safety factor and design pressure on wrappers and collars. 22

2.2 Relationship between safety factor and design pressure . . . . . . 23

3.1 Numerical results of collar plate reinforced specials. . . . . . . . . 50

3.2 Numerical results of wrapper plate reinforced specials. . . . . . . . . 51

3.3 Numerical results of crotch plate reinforced specials. . . . . . . . . . . 52

3.4 Parameters for models in Figure 3.20 . . . . . . . . . . . . . 53

3.5 Parameters for model in Figure 3.22. . . . . . . . . . . . . . 55

3.6 Parameters for models in Figure 3.27. . . . . . . . . . . . . 60

3.7 Parameters for wrapper and branch internal plate reinforced tee. . . 63

3.8 Parameters for models in Figure 3.20 . . . . . . . . . . 66

4.1 Test piece parameters with M11 design. . . . . . . . . . . . 68

4.2 Orientation of strain gauges. . . . . . . . . . . . 73

5.1 Strains at an internal pressure load of $1 \mathrm{MPa} \ldots \ldots . \ldots 85$

5.2 Strains at an internal pressure load of $2 \mathrm{MPa} \ldots \ldots . \ldots 85$

5.3 Sensitivity of simulated strains at an internal pressure of $1 \mathrm{MPa}$. . 86

5.4 Sensitivity of simulated strains at an internal pressure of $1 \mathrm{MPa}$. . 87

5.5 Strain comparison for internal and external surfaces . . . . . . . 88 


\section{Chapter 1}

\section{Introduction}

\subsection{Background to the Project}

Pipe specials (tees and lateral tees) are essential components in bulk water pipelines to provide a means of dividing and/or uniting flow(s). Figure 1.1 shows a typical tee (radial intersection) and lateral tee (non-radial intersection).

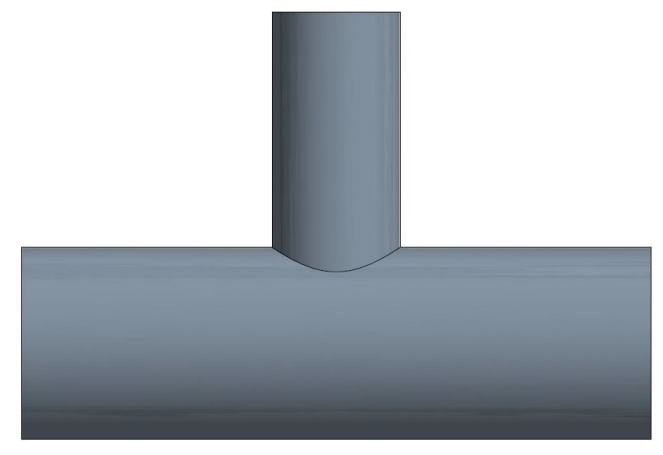

(a) Radial Tee

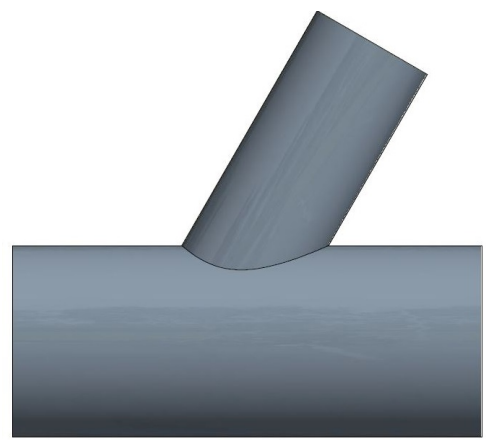

(b) Lateral tee

Figure 1.1: Typical plain ended radial (90 degree) and lateral tee.

These specials have a lower resistance to internal pressure than a pipe with equivalent size and thickness due to a loss of material in the sidewall of the main pipe, also referred to as the barrel or run pipe. Material is lost because a portion of the barrel is removed to allow for the branch to be welded to the barrel. A loss in material affects the stiffness of the special. Consequently, high local stresses arise at the intersection of these connections. Furthermore, longitudinal stresses arise in the throat of unrestrained specials from distortion and unbalanced hydrostatic pressure (AWWA M11). Steel reinforcements have been used on bulk water pipelines since the early 1900s. External reinforcement (stiffener) plates are frequently used to resist these high local stresses. A popular and internationally accepted design criteria for reinforcements of 
tees in bulk water supply pipelines is described in the American Water Works Association's (AWWA) M11 Manual. This is based on the research of Swanson et al. (1955) for the Department of Water and Power, City of Los Angeles. Figure 1.2 shows a typical two-plate, reinforced, 45 degree lateral tee manufactured in the early 1930s and published by Swanson et al. (1955). From the figure it can be seen that a large oval shaped section of the barrel is removed to allow for branching.
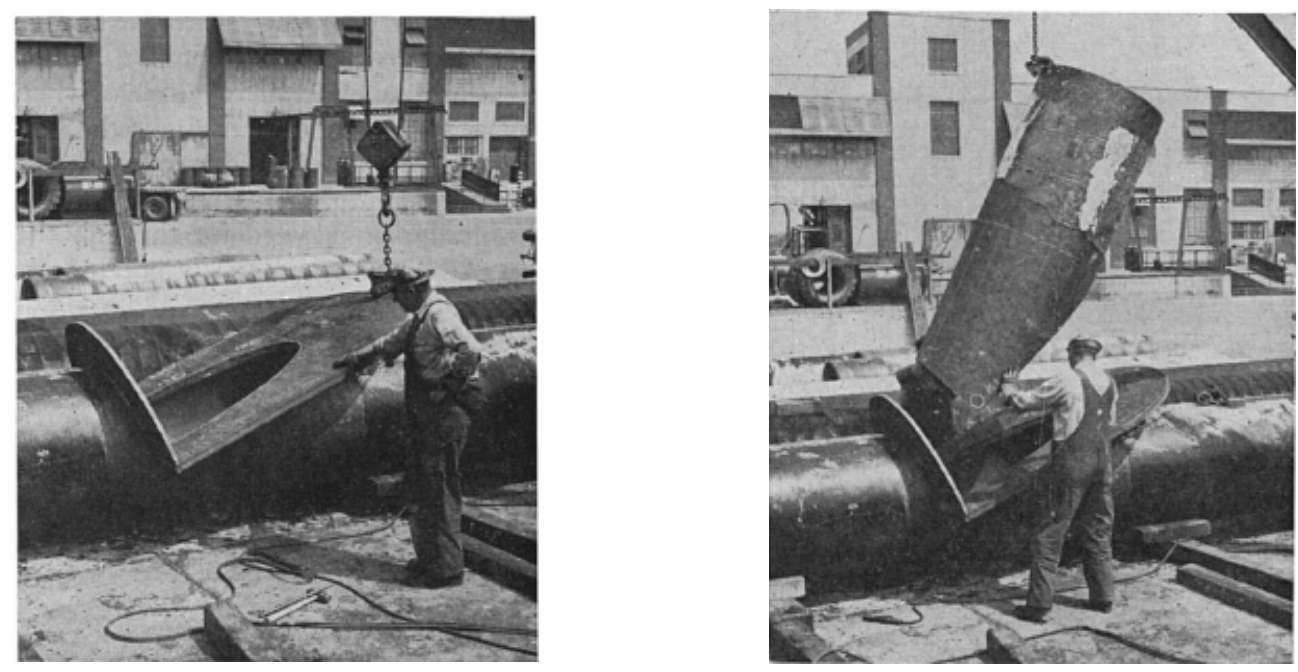

Figure 1.2: Two plate crotch plate manufactured in the early 1930s (Swanson et al., 1955).

The wall thickness of pipes commonly used for bulk water steel pipelines is generally much greater than required by minimum design theories for acceptable safety factors. As a result it is generally found that the lower safety factor of these specials, with the same wall thickness as the straight pipe they are connected to, remains adequate for general purposes (AWWA M11).

However, with the ever increasing demand for major pipelines to be constructed at a lower cost and with less material for the same operating pressures, it has become necessary to optimise the design of these specials. In addition, the design of reinforcements required for thinner walled specials must be optimised. Figure 1.3 shows the most common external reinforcements used on larger diameter steel pipe specials.

The development of higher grade steels has the potential to greatly reduce the need for reinforcements and provide engineers with more design options. The research by Swanson et al. (1955) is mainly based on experimental data obtained during tests on various specials. With the development in $3 \mathrm{D}$ computer aided design (CAD) and Finite Element (FE) analysis software, together with the development of higher strength steels over recent years, the following question arises and forms the motivation for this study: Can steel pipe specials for bulk water pipelines be designed with less reinforcing 


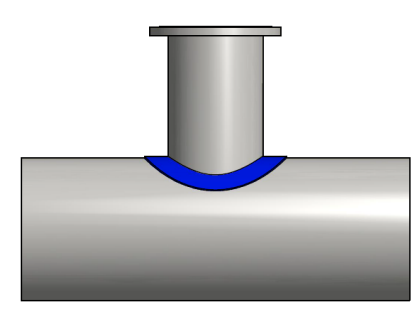

(a) Collar plate

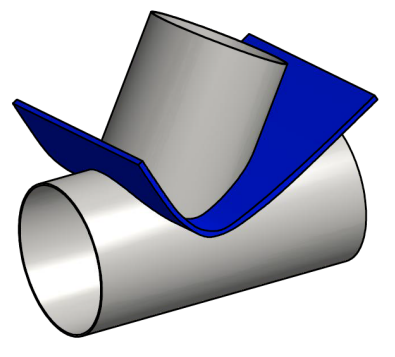

(b) One plate crotch plate

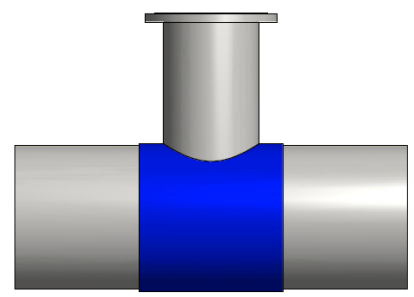

(c) Wrapper plate

Figure 1.3: Types of reinforcing plates for tees.

material using modern design tools such as $3 \mathrm{D}$ modelling and FE analysis? The purpose of this study is not to replace the commonly used design codes, specifically the AWWA M11 model, but rather trying to better understand where the code is accurate and where it is overly conservative. This is done to determine where improvements to reinforcements and the types of reinforcements would make sense.

Specials used in steel pipelines are often modelled with 3D CAD software, such as Inventor, for dimensional approval by professional engineers prior to manufacturing thereof. Figure 1.4 shows an isometric view of a typical 3D CAD model of a 45 degree lateral tee with a two-plate crotch design. Various CAD packages have integrated stress analysis (FE based) packages and with specials already modelled as part of the design process it becomes a relatively simple task to perform a stress analysis on such models. The following question could, however, rightly be asked: Are the integrated stress analysis packages built into market-leading CAD software, such as Autodesk Inventor, sufficient for the design of steel special reinforcements? Stand alone FE packages allow the user more modelling freedom, but also requires additional expertise and is generally associated with extra costs. 


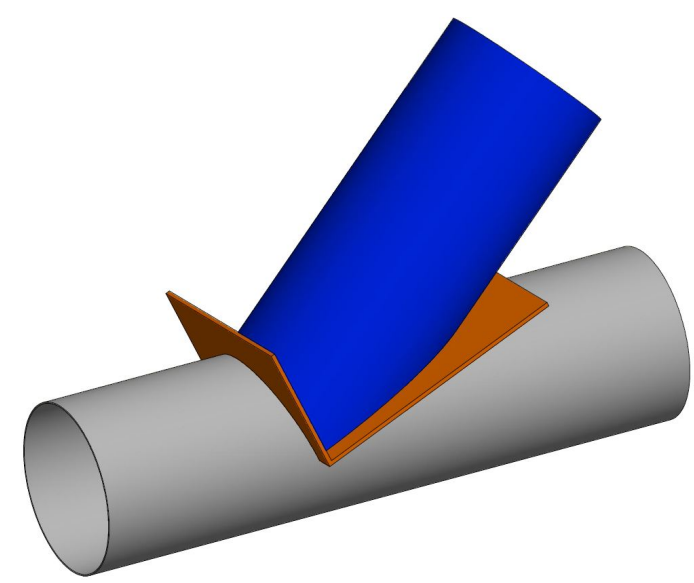

Figure 1.4: Typical 3D CAD model of plain ended lateral tee with two-plate crotch design.

\subsection{Objectives}

The main objective of this study is to evaluate the experimental-based design model provided by the AWWA M11 manual through comparison with numerical analyses. Specific attention is given to 45 degree lateral tees and numerical analyses are done on crotch, collar and wrapper reinforced tees. One lateral tee with a different intersection angle is also considered and an introductory analysis of reinforcements not in accordance with AWWA M11 are performed.

Further objectives, in the order of priority, are:

- Perform strain gauge measurements and visual checks on a hydrostatically loaded, reinforced, 50 degree lateral tee to validate the FE procedure followed in this study and verify simulated results.

- To analyse simulated results, obtained from both CAD-integrated FE packages and standalone FE software, as modern alternatives to analytical and experimental design methods, specifically the AWWA M11 model that is commonly used in the design of reinforcements for steel pipe specials. This objective deals with the effectiveness and suitability of CAD-integrated FE packages compared to standalone FE software. The suitability and effectiveness of these two approaches are compared with design standards such as the AWWA M11 model.

- Consider the effect of adjustments to the AWWA M11 model on failure loads and resulting safety factors, specifically the crotch plate design method, with special consideration to the mechanical properties of modern steels. 


\section{Chapter 2}

\section{Literature Study}

\subsection{Mechanics of Steel Pipe Specials}

Specials for steel pipelines are manufactured by removing a portion of the side wall of the barrel and welding on a branch pipe. The removal of material from the side wall of the barrel results in high local stresses at the edge of the hole. Attwater et al. (1994) pointed out that the high stresses is a direct result of self-equilibrating, discontinuous shear forces and moments which exist to maintain compatibility at the juncture. These forces, together with membrane forces that resist the internal pressure loading, give rise to the high local stresses. Welding and welding defects cause further weak points at the intersection region. Stress concentration factors are used to quantify local stresses in the vicinity of some stress raising feature. The stress concentration factor for an elliptical hole in an infinite flat plate under uni-axial loading is given by Equation 2.1.1. A typical uni-axially loaded infinite plate with an elliptical hole is shown in Figure 2.1.

$$
K_{t}=1+2\left(\frac{a}{b}\right)
$$

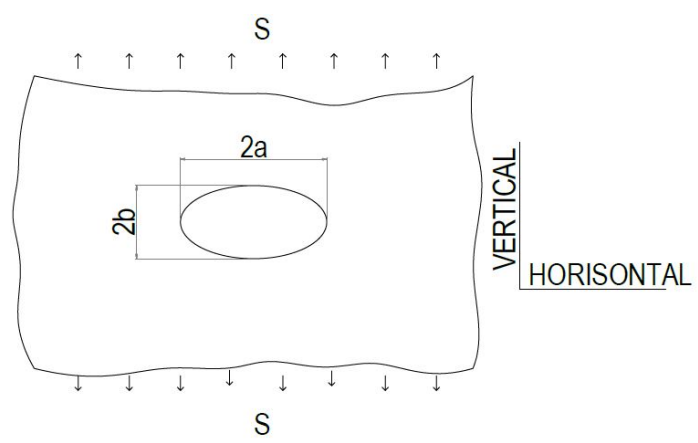

Figure 2.1: Elliptical hole in uni-axially loaded infinite flat plate. 
The tangential stress acting on the horizontal axis at the side of an elliptical hole through a plate (as shown in Figure 2.1) is given by Stephenson (1979) as:

$$
S^{\prime}=S\left(1+2\left(\frac{a}{b}\right)\right) \text { or } S^{\prime}=S K_{t}
$$

where

$$
\begin{aligned}
S^{\prime} & =\text { Tangential stress } \\
S & =\text { Uniform vertical (uni-axial) stress applied to plate } \\
a & =\text { Horisontal axis length } \\
b & =\text { Vertical axis length }(a=b \text { for circular holes) }
\end{aligned}
$$

From equation (2.1.2) it can be seen that the tangential stress is three times the applied stress for circular holes (as shown in Figure 2.2). It is important to note here that non-circular holes have higher stress concentration factors and hence higher local stresses occur at the edge of the hole. Likewise, the stress in a pipe shell, as a result of branching, is a maximum nearest to the branch/barrel intersection. Subsequently, reinforcements must be designed to provide the necessary support against these high local stresses.

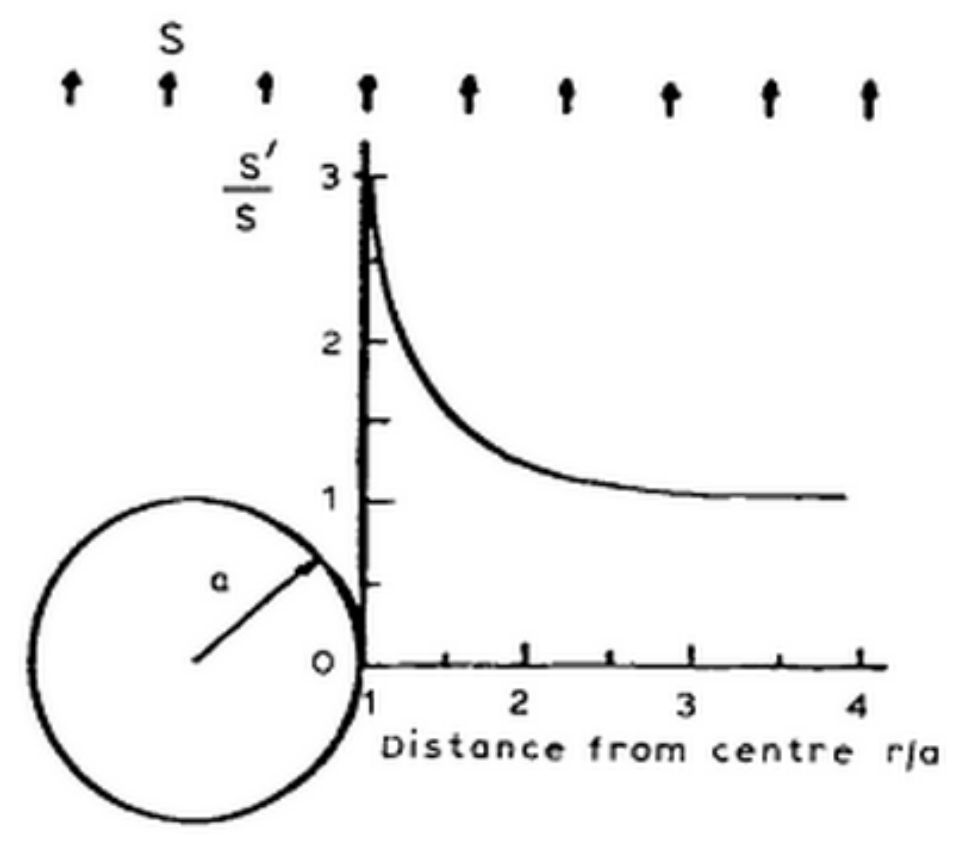

Figure 2.2: Stress distribution in a plate resulting from a hole (Stephenson, 1979). 


\subsection{Introduction to Stress Concentration Factors}

Stress concentration factors, for use in pipe specials, are defined as the maximum elastic stress in the intersection, divided by the maximum membrane stress. The maximum elastic stress is typically defined using the Von-Mises stress criteria and the maximum membrane stress is taken in the shell of the barrel or the branch. The hoop stress is typically used for the maximum membrane stress, because it is higher than the longitudinal stress in a straight pipe. Various studies have been performed over recent years to determine stress concentration factors (SCFs) resulting from cylinder/cylinder intersections. A large number of these studies have their origin in the pressure vessel environment. As a result, the branches (or nozzles as referred to by the ASME Boiler \& Pressure Vessel Code, Section VIII, Division 1-3) are significantly smaller than the barrel (vessel). Nozzles are typically small diamater stubs on pressure vessels that serve as inlets, outlets or sampling points. The highest stresses occur at the branch/barrel/reinforcing intersections and therefore the behaviour of steel at these intersections is of great importance for this study. Dekker and Bos (1997) noted that the maximum stress, resulting from internal pressure, is normally limited to $3 f$, where $f$ is the design stress or $2 / 3$ of the yield strength. Therefore, the maximum stress, according to Dekker and Bos (1997), is limited to twice the yield strength. This approach is based on elastic structural design and thus does not allow yielding for a safety factor of 2 .

The area replacement method has proven to be generally sufficient in the design of nozzles for pressure vessels or steel specials used in bulk water pipelines, where the diameter ratio (diameter of branch divided by diameter of barrel, $d / D$ ) is smaller than 1 . Extensive research on a cylindrical shell intersection with an intermediate diameter ratio $(d / D=0.526)$ has been done by Sang et al. (2002) who showed experimentally and with FE methods that the local stresses occurring at the barrel/branch intersection are typically in excess of the yield strength of the pipe material. This shows that local yielding occurs in the intersection region without necessarily resulting in failure of the pipe special (cylinder-cylinder intersection). It was further shown by Sang et al. (2002) that the burst pressure of this pipe special, under internal pressure, is lower than the theoretical burst pressure of the cylinder as calculated by the hoop stresses, resulting from an internal pressure loading. This is expected with the weaker intersection region. Little research could be found for stress concentration factors in large diameter ratio pipe specials.

Equal diameter specials are frequently used in bulk water pipelines and SCFs for such specials are typically significantly higher. This necessitates the need for reinforcements designed by methods capable of dealing with these high SCFs, as the area replacement method has shown to be insufficient in critical 
applications (such as high pressure and equal diameter intersections) as a result of higher stress concentration factors. It was concluded by Dekker and Bos (1997) that, in general, the stress intensity resulting from internal pressure, in comparison with other loads, absorbs the majority of the overall SCF. Thus, when using elastic structural design theory it is critical to estimate the stress concentration resulting from internal pressure as accurately as possible. SCFs are affected by, amongst others, the following parameters:

- $\beta$ - Diameter ratio, $d / D$

- $\alpha_{1}$ - Relative thickness of the barrel (wall thickness of barrel divided by diameter of barrel)

- $\alpha_{2}$ - Relative thickness of the branch (wall thickness of branch divided by diameter of branch)

- $t / T$ - Thickness ratio (branch thickness divided by barrel thickness)

Non-radial intersections (lateral tees) present significant difficulties due to the lack of symmetry of the cut-out in two planes when compared to radial intersections. Lekkerkerker (1972) pointed out that, when the branch pipe is of nearly equal diameter to the intersected cylinder, the curve of the intersection is not nearly a circle in the developed shell surface. This is illustrated in Figures 2.3 and 2.4. It is clear from these flat patterns that SCFs for equal diameter and non-radial intersections will be significantly higher than for circular or near-circular holes, as shown in Figure 2.2 and equation 2.1.2. This is further supported by the elliptical shape of a section through the barrel/branch intersection as shown in Figure 2.5. 


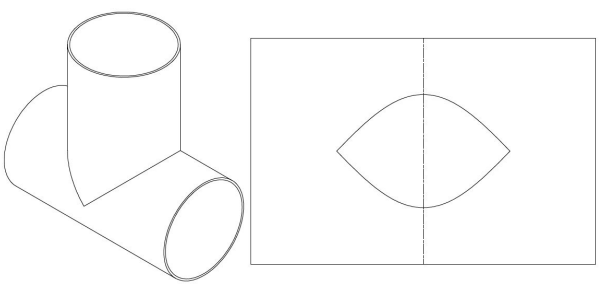

(a) Equal diameter tee

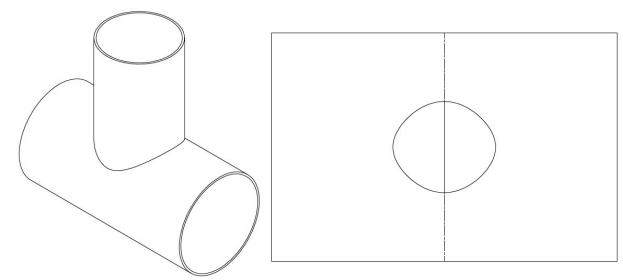

(b) Unequal diameter tee

Figure 2.3: Typical barrel flat patterns for radial intersections.

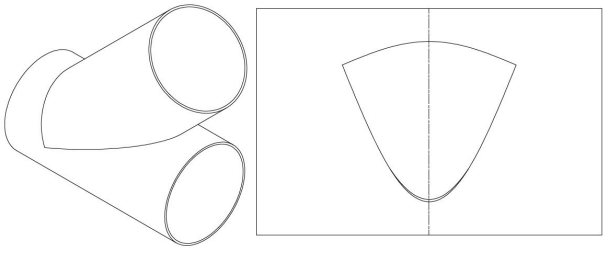

(c) Equal diameter tee

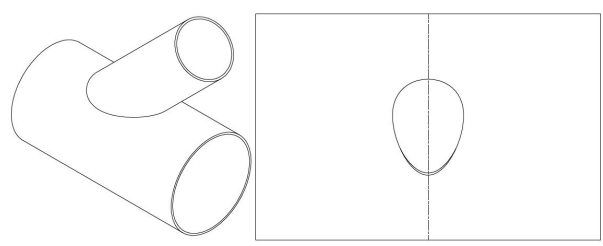

(d) Unequal diameter tee

Figure 2.4: Typical barrel flat patterns for non-radial intersections.

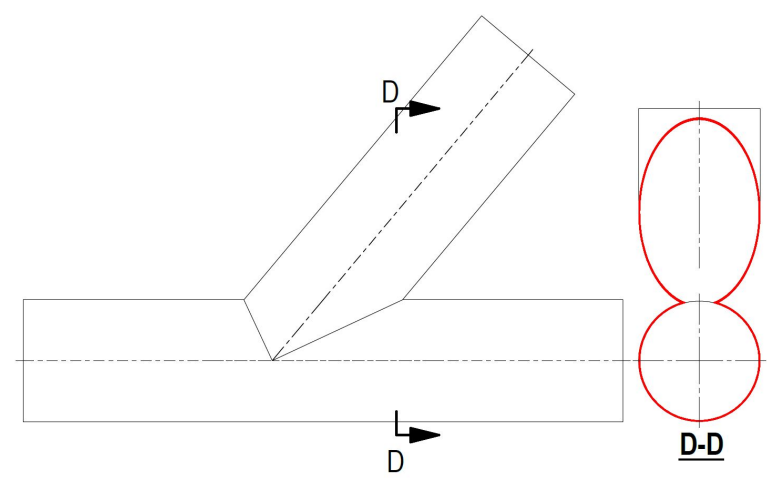

Figure 2.5: Elliptical shape of branch/barrel lateral tee section

SCFs also increase significantly with a decrease in the intersection angle. The highest stresses develop typically from the inside (acute) crotch corner shown in Figure 2.6.

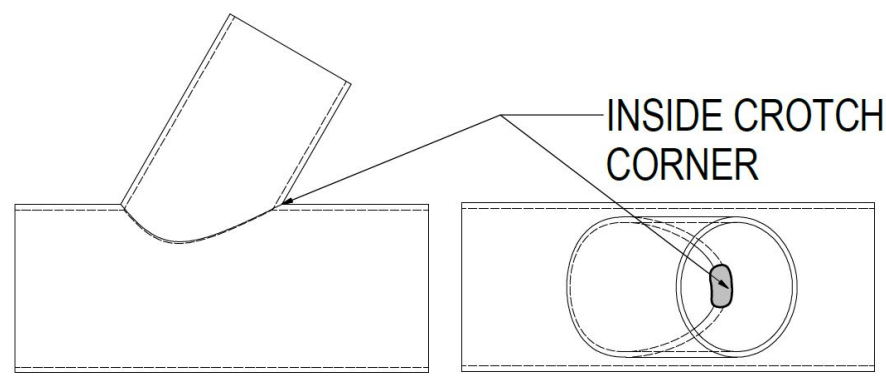

Figure 2.6: Inside crotch corner of non-radial intersection. 
Distinction is made by Dekker and Bos (1997) between primary and secondary stresses in specials with membrane stresses resulting from both internal pressure and external loads defined as primary stresses. Bending stresses due to both internal pressure and external loads are classified as secondary stresses. According to Dekker and Bos (1997) the sum of the primary and secondary stresses must be considered to prevent low cycle fatique and it is therefore recommended that combined SCFs be determined by considering both primary and secondary stresses. Bulk water pipelines are, however, not generally subjected to external loads and hence stress concentration factors are based only on internal pressure loads. External loads are normally restrained by either pipe supports or backfill material in buried pipelines.

Previous studies, by amongst others Dekker and Bos (1997) and Dekker and Stikvoort (1997), have presented various analytical and numerical SCF calculation methods and SCFs for various branch and barrel intersections have been developed by Dekker and Bos (1997). The modified improved shrink ring (MIST) method was used to determine the external load stress intensities while the internal pressure stress intensities were determined by FE methods. Dekker and Stikvoort (1997) pointed out that there are substantial differences between the various design codes and concluded that SCFs obtained numerically with FE methods appear to give more reliable results than the analytical methods. The latter is confirmed in previous work done by Dekker (1993), specifically for pressure vessels and thick-walled cylinders used in the offshore oil industries. Dekker and Brink (2000) added that the use of quadrilateral plate/shell elements in FE analyses suffices in providing useful SCFs. This despite the fact that there is partial overlap of the nozzle (branch) and shell (barrel) at the junction and not accounting for the additional reinforcement area of the weld's throat thickness, as shown in Figure 2.7.
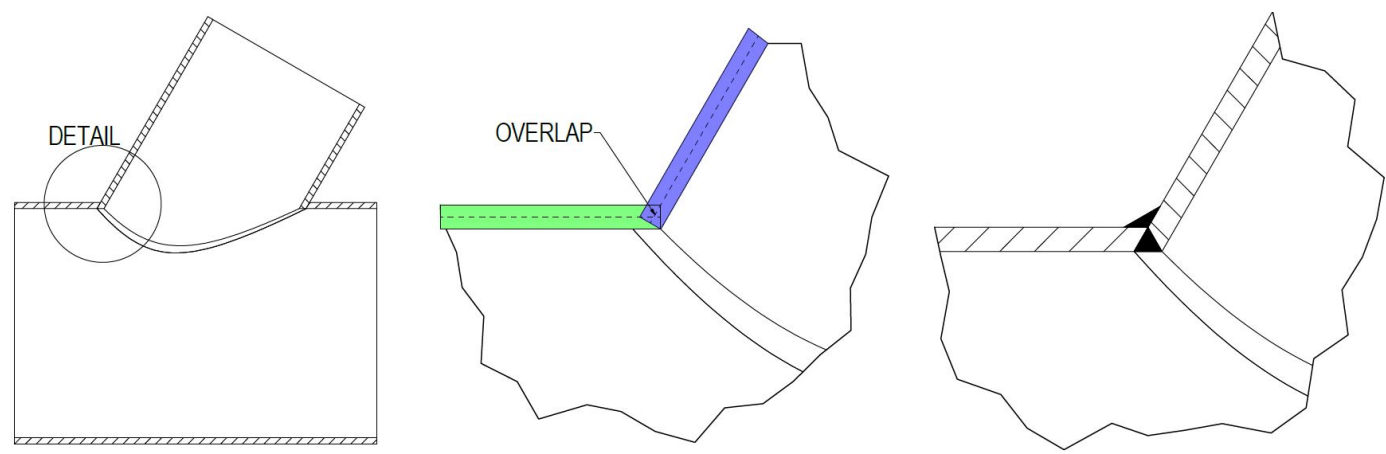

Figure 2.7: FE modelling of specials with shell elements.

Dekker and Brink (2000) concluded that the additional reinforcement area at the barrel/branch intersection due to the weld's throat thickness have little influence on the maximum allowable internal pressure and hence does not influence the SCF at the intersection. They further advised to not exploit all 
pressure capacity of the specials as a sufficient stress margin should be left to deal with external loads such as thermal expansion of connecting pipes etc. The effect of reinforcing on SCFs was investigated by Finlay et al. (2003), who concluded that there appears to be no obvious relationship between SCFs and increasing diameter ratios for reinforced tees. SCFs are therefore not considered to be a factor of the diameter ratio, but rather of material, intersection angle and other geometrical discontinuities. Their study did, however, conclude that the SCFs for unreinforced fabricated tees proved to be higher than for reinfoced tees. This correlates perfectly with the idea that reinforcing is to be added to barrel/branch intersections for stiffness and strength. Qadir (2008) pointed out that there is a direct relation between an increase in wall thickness and a decrease in the high stresses occurring at the crotch corner of cylinder/cylinder intersections. Stress intensities increase as the barrel or branch wall thickness decreases as it results in a loss of stiffness.

SCFs only seem to make logical or practical sense when defined for the linear elastic region. In this study the steel is allowed to yield locally and not necessarily considered to have failed when local yielding occurs (refer to Section 3.5.1) as the special's load-carrying capacity is not exhausted when yielding impends. This observation is supported by Sang et al. (2002) who pointed out that, when using the limit design method instead of elastic structural design, small local yielding may occur in the intersection region under the allowable pressure. The allowable pressure, according to this design method, is two thirds of the limit load. Cook and Young (1999) also pointed out that, for a given safety factor, the limit design method allows a greater working load than the elastic design approach. Once yielding occurs and nonlinear behaviour presents itself, SCFs cannot be calculated on the same basis as described above. In particular when it is assumed that the material is elastic perfectly plastic (EPP) i.e. the maximum stress is capped at the yield strength of the steel. In this case the SCF cannot increase further, provided that the membrane stress does not reduce. However, in reality yielding does develop further with an increase in internal pressure and as a result the factor of safety reduces.

By limiting the validity area of SCFs to regions that are sufficiently far away from the high stress region(s), so that the stresses fall in the linear elastic range, SCFs could still be used as a design criteria. However, insufficient literature seems to be available as to how such valid areas, in the vicinity of high local stresses, are to be determined for large diameter steel pipe specials. Increased yielding, as a result of increased loads, will push these areas (where SCFs remains valid) even further away from the originating high stress zones. SCFs are therefore not considered to be valuable as a design criteria where stresses are expected and in fact allowed to reach the steel's yield strength, with the assumption that the effects of fatigue need not be considered. Instead, alternative design tools, such as numerical models, are proposed for steel pipe specials. Numerical tools, such as FE analysis, can assist the designer in identifying high stress zones and determining safety factors. Nevertheless, the 
use of stress concentrations remain valuable in identifying high stress regions.

\subsection{Reinforcement of Specials for Steel Pipelines}

The aim of this study is not to describe all available reinforcement methods in detail, but rather to evaluate reinforcements contained in the AWWA M11 design model, as described in Section 1.2. An overview of these and other common reinforcement methods and design models is, however, justified and hence presented here.

The use of crotch, collar and wrapper plates (as shown in Figure 1.3) are well-established reinforcing methods for specials in large diameter steel pipelines operating at high internal pressures, typically in excess of $1 \mathrm{MPa}$. Wrapper and collar plates are generally used for specials with diameter ratios less than 0.7 and lower design pressures. The area replacement method, described further in Section 2.4.2, is generally used for the design of collar and wrapper reinforcements. Crotch plates on the other hand are used for higher design pressures and equal or near-equal diameter specials. The AWWA M11 model is commonly used for the design of crotch plates and relies on experimentally obtained data in the form of graphs. Figure 2.8 shows a typical tee with collar plate reinforcing. The collar plate is typically welded to the barrel and branch by means of fillet welds.

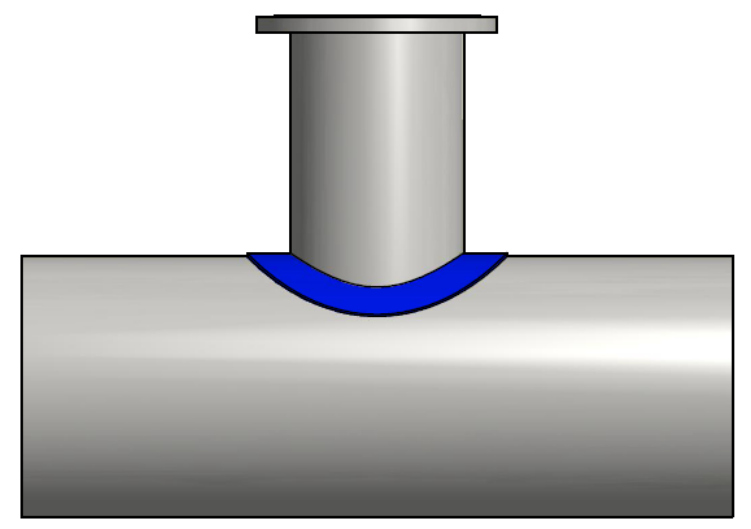

Figure 2.8: Typical tee with collar stiffener plate.

Wrapper plates (shown in Figure 2.9) are similar to collar plates, with the difference that it covers the entire circumference of the barrel. The plate is welded to the barrel and around the branch pipe at the intersection of the branch and barrel. 


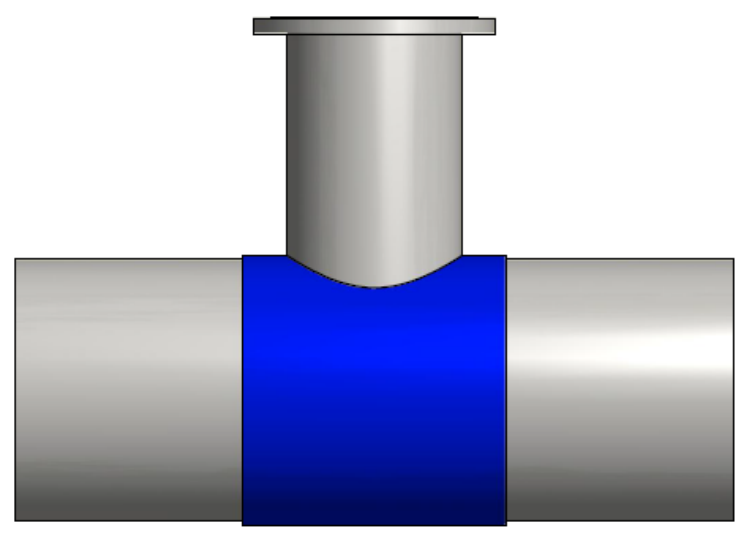

Figure 2.9: Typical tee with wrapper stiffener plate.

According to Swanson et al. (1955), the Swiss firm Sulzer Brothers first proposed a circular collar reinforcement for lateral tees in 1928 and the crotch plate design developed from this early work. Swanson et al. (1955), amongst others, contributed greatly to the crotch plate design as a reinforcement method for tees and wyes (Stephenson, 1979). Over the years the model proposed by Swanson, as contained in the M11 manual, has been widely accepted for the design of reinforcements of bulk water steel pipe specials. An alternative, yet similar to AWWA M11, design model is presented by the British Standard Institution (EN13480-3) for 90 degree tees. Various area replacement type formulations for reinforcements are proposed by this standard. For diameter ratios smaller than or equal to 0.8 the opening can be reinforced by either integral thickening of the barrel wall, branch wall or the addition of set-on welded reinforced pads (wrappers and collars). These methods are presented graphically in Figure 2.10.

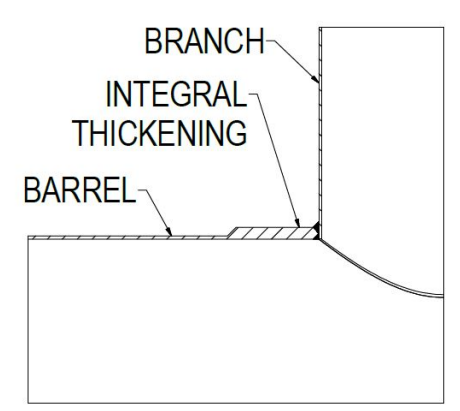

(a) Barrel thickening

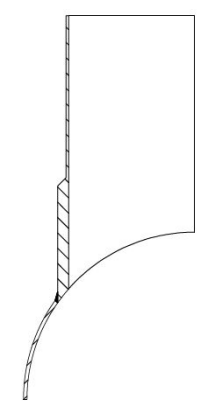

(b) Branch thickening

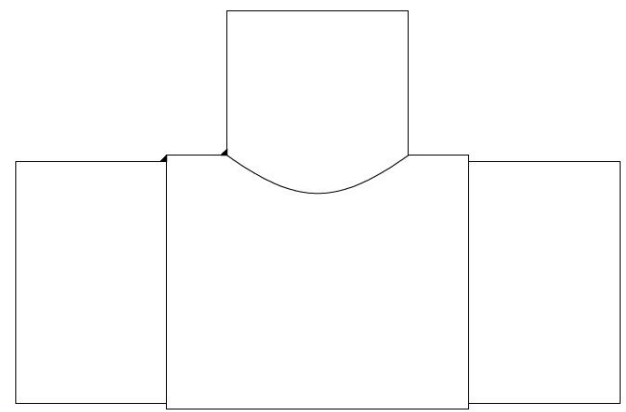

(c) Reinforcing pad (wrapper)

Figure 2.10: Reinforcement methods presented by EN13480-3. 
It was noted by McIntyre et al. (1977) that several theoretical and experimental studies have been made which show considerable differences in the elastic stress distributions of integral and pad reinforcements. The EN13480-3 design model differentiates between these two type of reinforcements. AWWA M11, however, does not specifically include for the design of integral reinforcements. The EN13480 standard also allows for the use of reinforcing pads on diameter ratios larger than 0.8 under condition that the design is outside the creep range of the pipe material. The difference in safety factors between integral and pad reinforcements is discussed further in Section 3.8.

The EN13480 standard further provides a design model for three-plate crotch plates on lateral tees, as shown in Figure 2.11. The stabilizer bar provides further stiffness to the intersection region. The analytical foundation for these formulations are however not mentioned and hence a conclusion on the factor of safety, provided by designs in accordance with this code, could not be reached. The special shown in Figure 2.11 was analysed numerically and the results are presented in Section 3.9. For the purpose of this study, only the AWWA M11 model is described in further detail in Section 2.4.

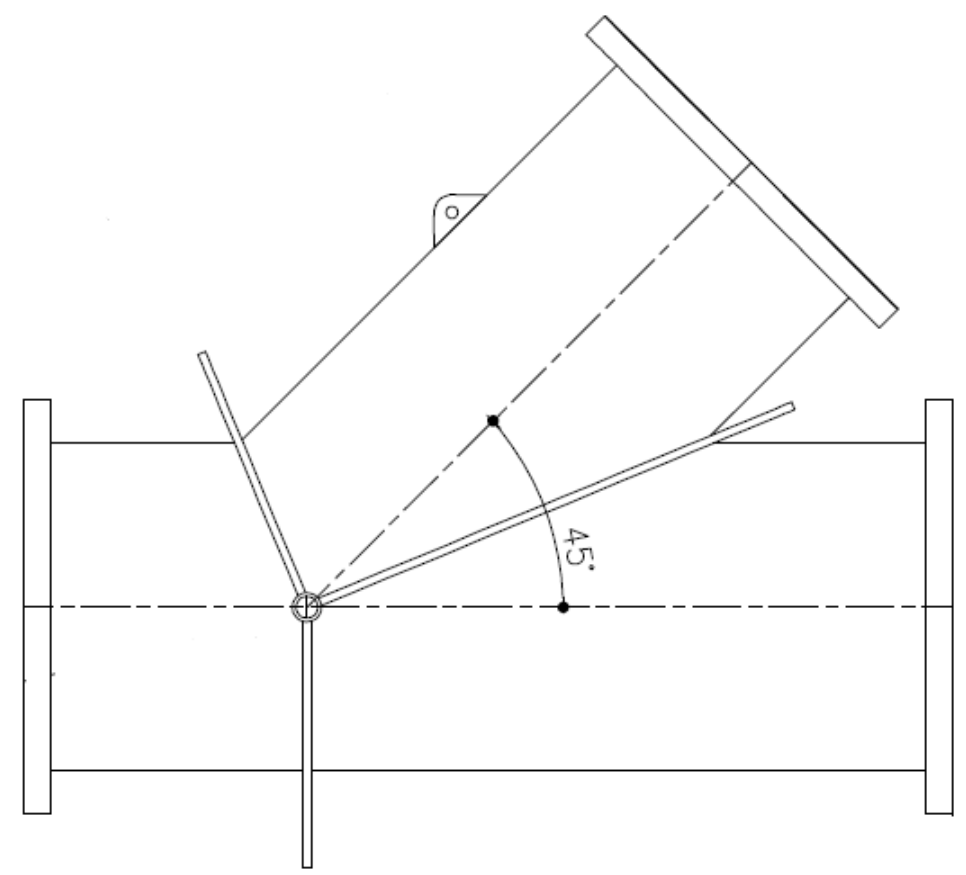

Figure 2.11: Typical crotch plate design in accordance with EN13480-3.

Figure 2.12 shows typical two and three plate reinforcing tees. Both crotch plates are welded to the barrel, the branch and their ends welded to each other. The third plate (ring plate/back plate) is welded only to the other two plates (crotch plates) with clearance between the ring plate and the barrel shell to prevent the transformation of shell stresses to the ring plate. Crotch 
plates provide stiffness to specials by acting as a clamp around the barrel and therefore reduce outward deformation

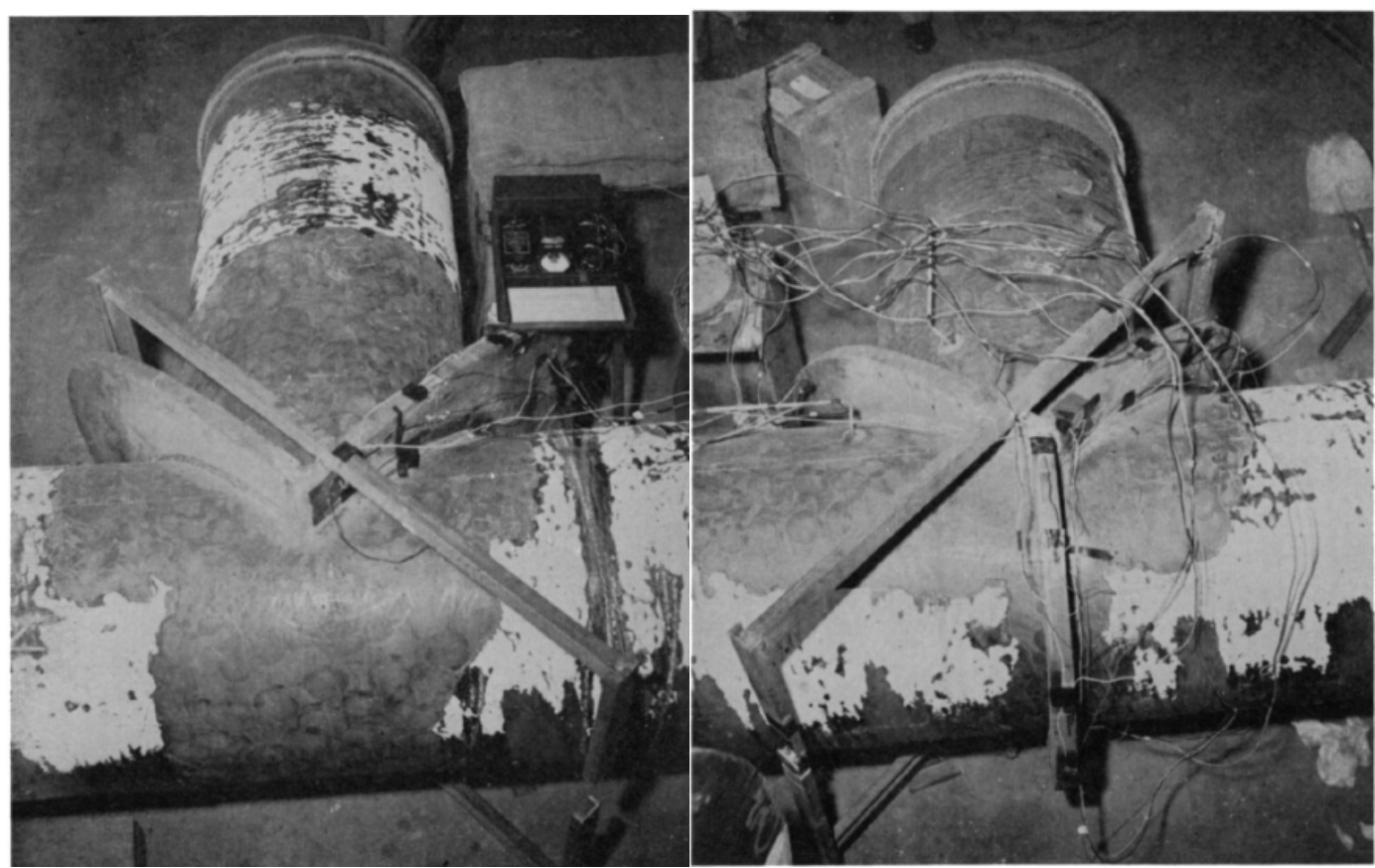

(a) Two-plate design

(b) Three-plate design

Figure 2.12: Two and three plate tees tested with strain gauges (Swanson et al., 1955).

A photo of a two-plate, unequal diameter, crotch plate reinforced tee, installed at Kumba Iron Ore (Sishen) Mine, near Kathu in the Northern Cape province of South Africa, is shown in Figure 2.13. It can be seen that this design uses two straight plates welded to the intersection instead of a single curved plate normally used on unequal diameter tees. Additional reinforcing plates were also used between the crotch plates and the barrel to provide further stiffness.

Ring plates are recommended for DN 1500 (Diamater Nominal) or larger specials (Swanson et al. (1955)). Diameter Nominal (DN) is used in the metric unit system for standard pipe sizes and is an indication of the diameter of the pipe. The nominal diameter is close to, albeit not exactly, the outside diameter of a pipe and is derived from the imperial unit system. The design of stiffener plates must take into account factors such as localised structural discontinuities, working and design pressures, restraints on stiffener plates, pipe supports, surge conditions, mechanical properties of steel, temperature, branch deflection angle (angle of branch/barrel intersection) and safety factors. Other factors may include dead loads and erection procedures, although these 


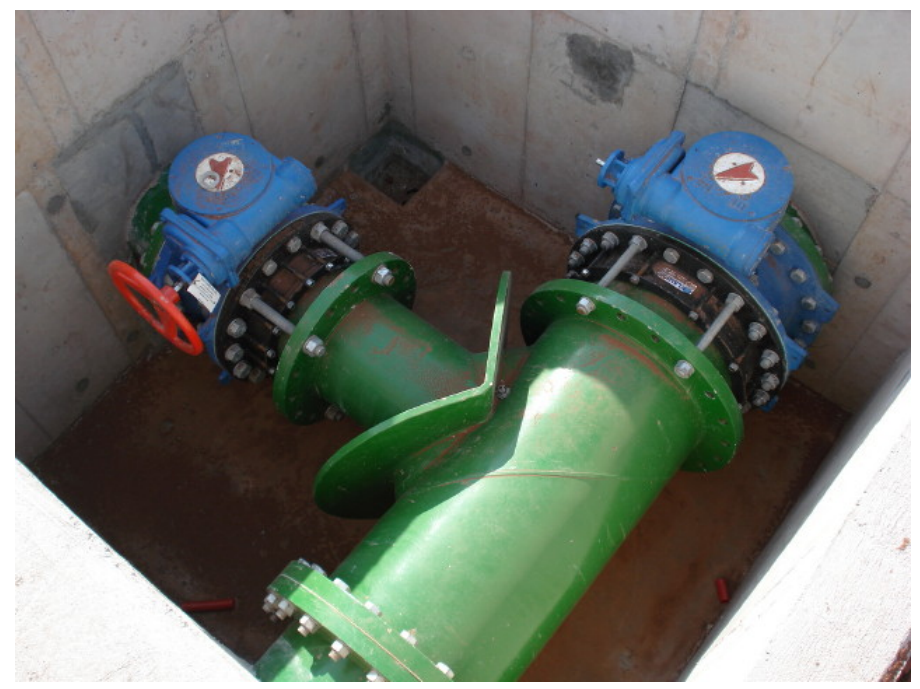

Figure 2.13: Two plate crotch plate installed at Sishen Mine (Aurecon).

are typically negligible. Smaller deflection angles require larger elliptical cutouts in the barrel and subsequently results in additional material losses.

Determination of the loads imposed on the resisting members and parts of the structure that resist these loads is the major task in the structural analysis (Stephenson, 1979). The resisting members (stiffener plates) are subjected to bending, tension and shear stresses as a result of the load applied by the pipe shell under hydrostatic loads. The effects of external loads on these pipe specials are disregarded in this study as it is assumed that the adjacent pipework connected thereto are fully restrained and hence no external loads are transferred to the pipe specials. This assumption is based on well-designed physical pipeline installations where pipework and specials as well as other fittings such as bends resulting in a change of flow direction and thus high resulting forces are not supported or restrained to adjacent pipework which would result in bending moments.

Internal bracing is an alternative reinforcing method and has been used successfully for decades. While external reinforcing plates provide stiffening mainly by bending, internal braces are subjected to pure tension and hence is more efficient. The compact arrangement of internal bracing facilitates easier transport and installations. Internal bracing could, however, act as a flow restriction and hence cause increased pressure losses, which could have significant impacts over the lifetime of the special. Extensive theory for the design of internal bracing is provided by Stephenson (1979). For very large diameter specials and high design pressures a combination of external and internal supports might prove to be optimal. Internal bracing is discussed further in Section 3.9. 


\subsection{AWWA M11 Design Model}

\subsubsection{General}

The M11 model covers the design of reinforcements for tees and single lateral tees (shown in Figure 1.1). The design of symmetrical tees (wyes), crosses and double laterals, however, falls outside the scope of the M11 model and additional analyses are therefore required for such specials. The model proposed by Swanson et al. (1955) is published in the M11 manual and dictates that reinforcements must be designed such that the design stress in the reinforcement is not greater than the hoop stress of the pipe. The M11 model makes use of two parameters in determining the appropriate type of reinforcement. For the purpose of this study all customary units, as given in the M11 manual, have been transferred to the SI unit system. The first parameter is the magnitude of the pressure diameter value (PDV) and calculated as follows:

$$
\mathrm{PDV}=\frac{P d^{2}}{D \sin ^{2} \Delta}
$$

where

$$
\begin{aligned}
P & =\text { Design pressure [MPa] } \\
d & =\text { Branch outside diameter }[\mathrm{mm}] \\
D & =\text { Barrel outside diameter }[\mathrm{mm}] \\
\Delta & =\text { Branch pipe angle of deflection [degrees] }
\end{aligned}
$$

The second parameter is the diameter ratio, defined as the ratio of the branch outside diameter to the barrel outside diameter $(d / D)$. The design of reinforcements becomes more critical with an increase in the branch diameter. When the magnitude of the PDV is smaller than 1050, the diameter ratio is used to distinguish between either wrappers or collars. Wrappers are used for diameter ratios larger than 0.7 whereas collars are used for ratios smaller than or equal to 0.7. Crotch plates are used for PDV values above 1050. The PDV values, as stated above, were determined by Swanson et al. (1955) through experimental analyses and are based on steel with a yield strength of approximately $207 \mathrm{MPa}$ (30 $000 \mathrm{psi}$ ). The yield stress of modern steels used for steel pipes are, however, typically in excess of $290 \mathrm{MPa}$.

Collars and wrappers are designed using the area replacement method while the nomograph design is used for crotch plates. The AWWA M11 design model is widely used in the bulk water industry and has proven to be a reliable model. Some drawbacks of this design model are listed below and have been the driving force of this study.

- It is based on steel with a much lower yield strength than what is typically being used today i.e. it is considered to be overly conservative. 
- Experimental tests were performed in the 1950s when testing equipment were not as good as they are today.

- It does not seem to effectively deal with the plasticity of steel.

\subsubsection{Collars And Wrappers: Area Replacement Method}

Wrapper and collar plates are designed to account for the loss of material in the barrel as a result of branching. Figure 2.14 shows a cross-section of a wrapper or collar reinforced tee as published in the AWWA M11 manual.

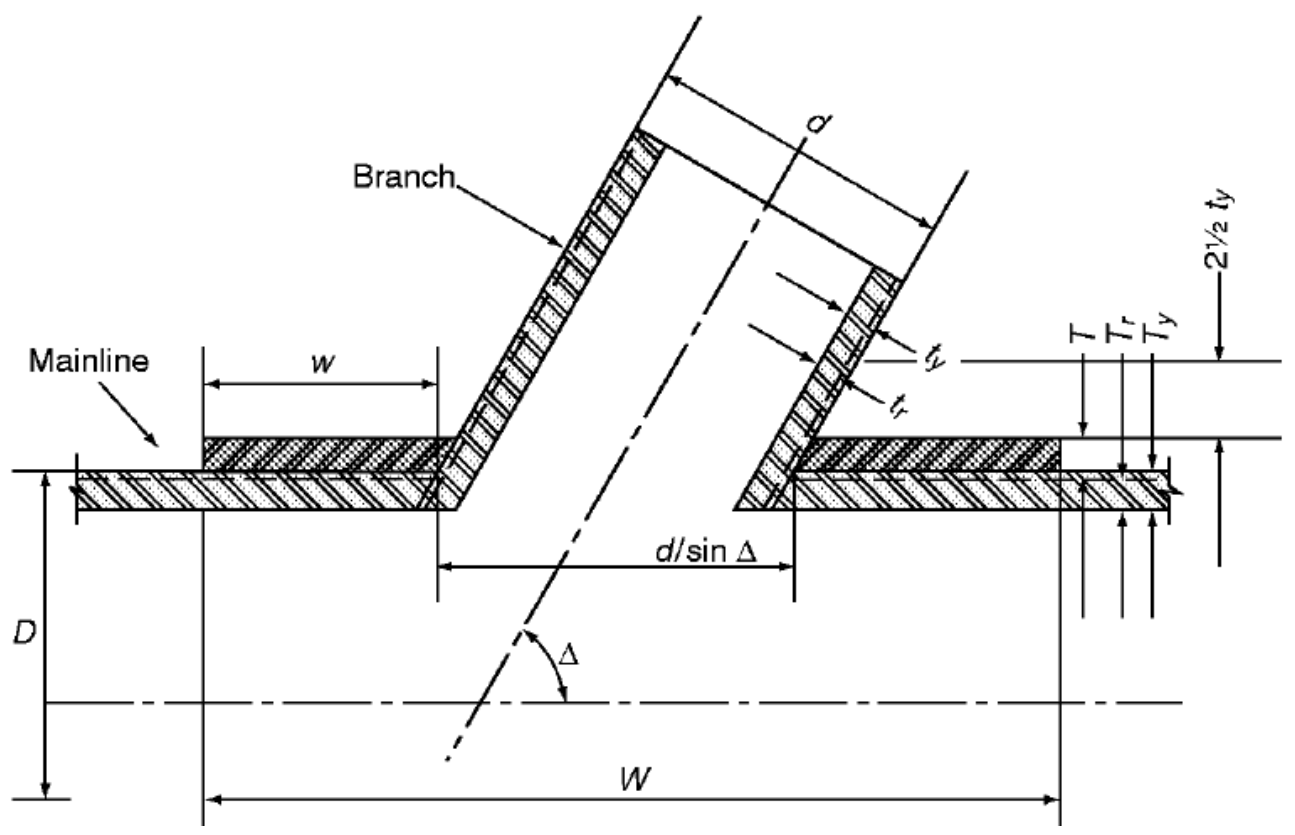

Figure 2.14: Collar or wrapper reinforcement of steel pipe special (AWWA M11) 
where

$$
\begin{aligned}
D & =\text { Barrel outside diameter } \\
T_{y} & =\text { Wall thickness of barrel } \\
T_{r} & =\text { Required min wall thickness of barrel, based on hoop stress } \\
d & =\text { Branch outside diameter } \\
t_{y} & =\text { Wall thickness of branch } \\
t_{r} & =\text { Required min wall thickness of branch, based on hoop stress } \\
T & =\text { Thickness of wrapper/collar } \\
W & =\text { Overall width of wrapper/collar } \\
w & =\text { Wrapper/collar edge width }
\end{aligned}
$$

The required minimum wall thickness of the barrel $\left(T_{r}\right)$ is based on the allowable stress in the shell:

$$
T_{r}=\frac{P D}{2 f_{s}}
$$

The maximum allowable stress (design stress, $f_{s}$ ) for collars and wrappers is limited to half the minimum yield stress of the shell or the reinforcement, whichever is less. This equates to a minimum recommended safety factor $(S F)$ of 2 , based on the yield stress of the material, an indication that the plasticity of steel is not accounted for. The design stress in the reinforcement should also not be greater than the design stress, typically hoop stress, used in the design of the straight pipe. Figure 2.15 shows a simplified cross section of a lateral tee with a wrapper (stiffener) plate. The stiffener plate is marked as 3 . The thickness of sections 4 and 5 together form the wall thickness of the barrel, with the thickness of section 5 being the minimum wall thickness required in the main pipe for the design pressure, based on hoop stress. Section 4 is therefore excess material in the wall of the barrel, not required to withstand internal pressure. Wall thicknesses commonly used in pipelines are often much greater than required by internal pressure conditions and hence the excess material denoted by section 4 .

Sections 1 and 2 denote the material removed from the barrel to allow for branching. The minimum area required in the barrel wall at the location of the hole to resist internal pressure is denoted by Section 1. Section 6 is defined as the allowable outlet area. The required reinforcing area is calculated as follows:

$$
A_{r}=A_{o}+A_{e}+A_{w}
$$




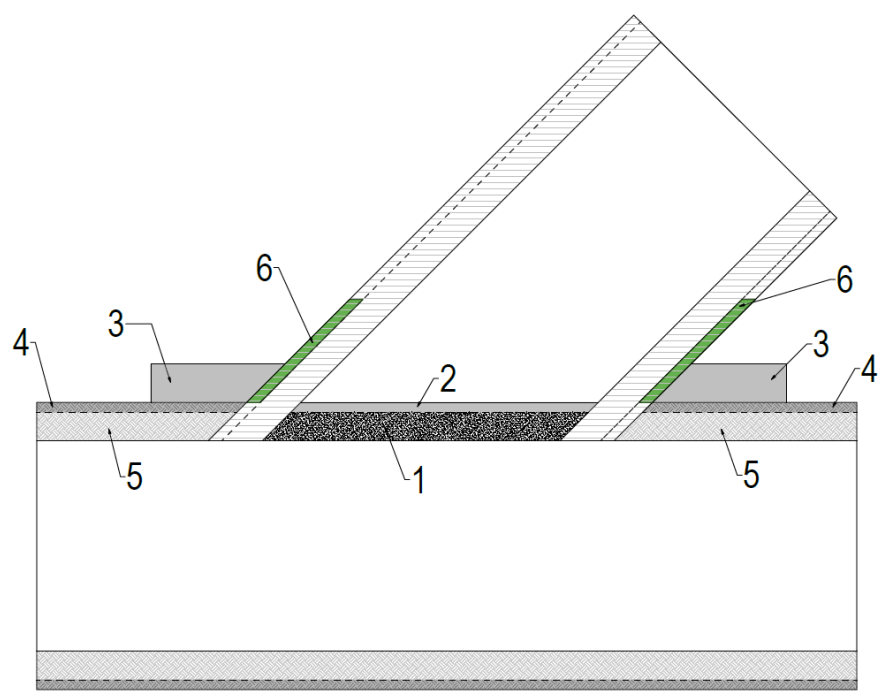

Figure 2.15: Simplified cross-section of a typical wrapper reinforced lateral tee.

where

$A_{r}=$ Theoretical minimum required reinforcing area removed from the barrel, section 1

$A_{o}=$ Available outlet area, section 6

$A_{e}=$ Allowable excess area, section 2

$A_{w}=$ Required reinforcement area provided by the wrapper, section 3

The reinforcing area removed from the barrel (shown as section 1 in Figure 2.15) is multiplied by a multiplier factor $(M)$. The multiplier factor is 1 for PDV magnitudes smaller than 700 and 0.00025 times PDV for PDV magnitudes between 700 and 1050. The AWWA M11 model does not provide information as to how the multiplier factor was obtained or the reason for the discontinuity at a PDV of 700 . The removed reinforcing area is calculated as follows:

$$
A_{r}=M\left(\frac{T_{r}\left(d-2 t_{y}\right)}{\sin \Delta}\right)
$$

The allowable outlet area (Figure 2.16) is the excess material in the branch that is considered to provide stiffness which is lost by the removal of material from the barrel, to the intersection.

The allowable outlet area is calculated as follows:

$$
A_{o}=2\left[2.5 t_{y}\left(t_{y}-t_{r}\right)\right]
$$

The wrapper plate area $\left(A_{w}\right)$ is shown in Figure 2.17 and the area is calculated by:

$$
A_{w}=A_{r}-A_{o}-A_{e}
$$




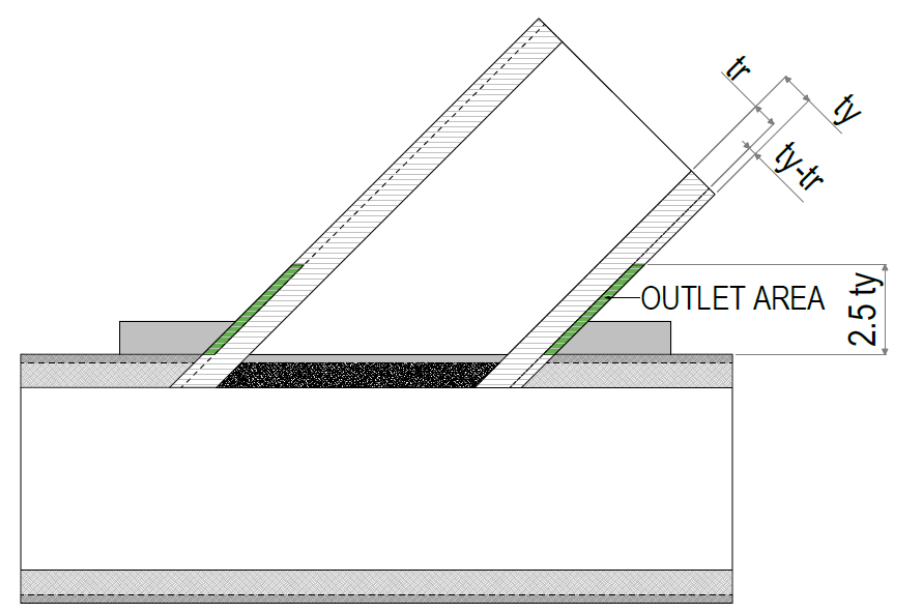

Figure 2.16: Allowable outlet area.

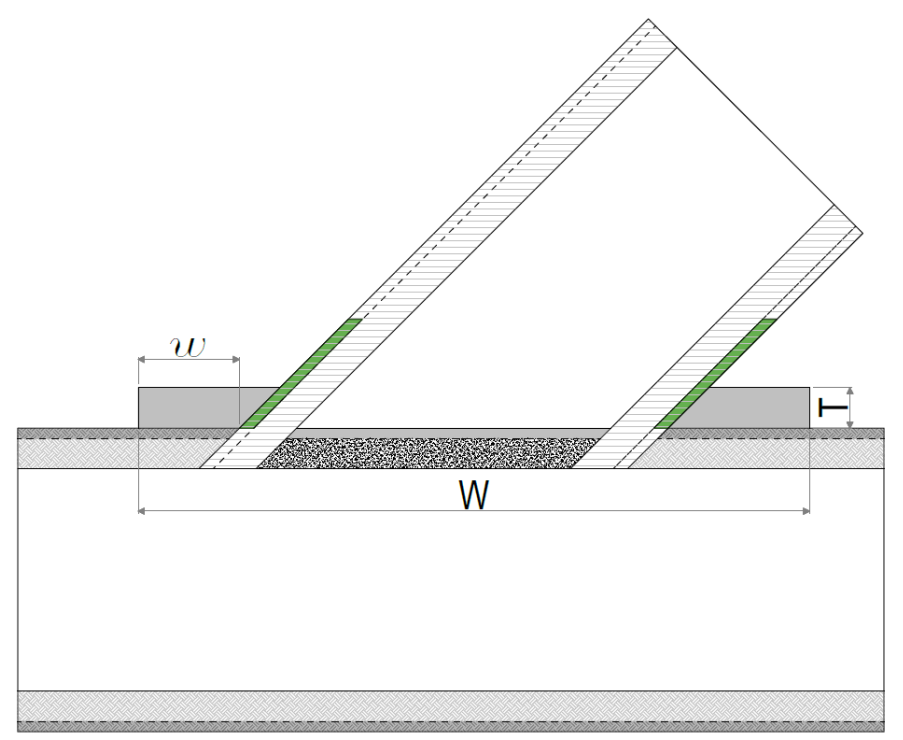

Figure 2.17: Wrapper plate cross-sectional area.

It should be noted from equation 2.4.5 that the length of the allowable area (i.e. $2.5 t_{y}$ ) is not a function of the deflection angle and hence the length of the outlet area does not increase with a decrease in deflection angle. The allowable excess material (shown as section 2 in Figure 2.15) is defined as the excess material in the shell of the barrel, which was removed by the branching and which is not required for internal pressure in the barrel. The cross-sectional excess area (as dimensioned in Figure 2.18) is calculated as follows:

$$
A_{e}=\left(T_{y}-T_{r}\right)\left(\frac{d-2 t_{y}}{\sin \Delta}\right)
$$

From the above formulas the minimum allowable width and thickness of 
the stiffener plate is calculated. The same equations apply to collar plates.

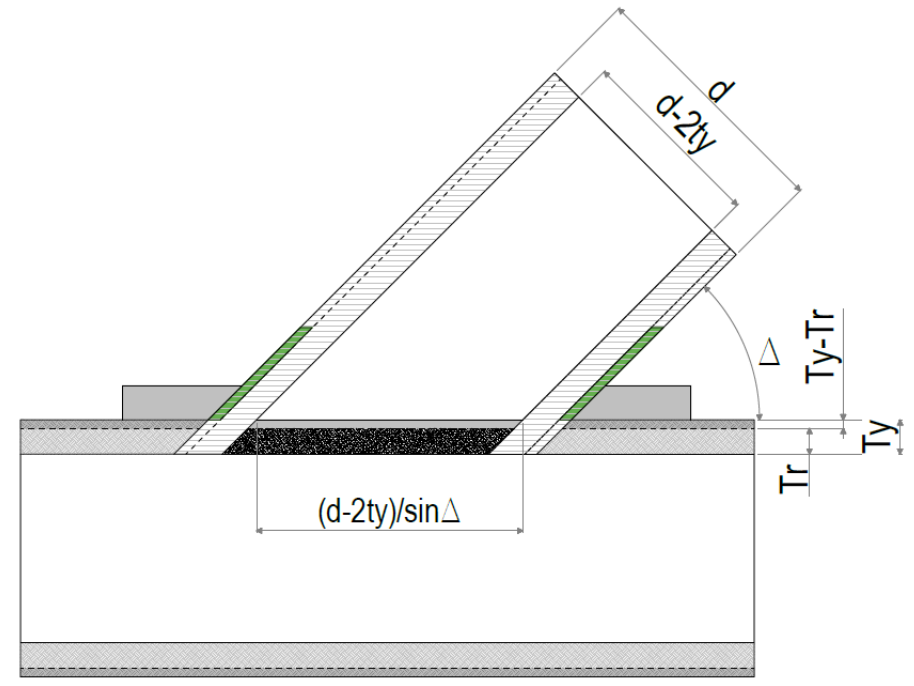

Figure 2.18: Allowable excess area.

Yield strength does not affect the PDV value. For wrapper and collar plate calculations it affects only the required minimum wall thickness of the barrel $\left(T_{r}\right)$. However, yield strength does affect the safety factor for a given design stress. To illustrate this two wrapper reinforced specials were chosen with the same wrapper dimensions. Cases 1 and 2 in Table 2.1 show the effect of a change in safety factor on the design pressure. The allowable yield strength is defined as the maximum yield strength to be used in the design of reinforcements in order to account for a factor of safety. A safety factor of two results in an allowable yield strength of half the actual yield strength of the material. It is clear that the design pressure $(P)$ is not directly related to the yield strength, i.e. increasing the safety factor by a factor of 2.325 does not reduce the design pressure by a factor of 2.325 . The resulting design pressure, referred to in Table 2.1 is the design pressure which, for the chosen safety factor, results in the size of wrapper/collar as indicated in the table. The size of reinforcement for case 1 and 2 is the same to allow for comparison between safety factors and design pressures. Cases 3 and 4 in Table 2.2 show that a reduction in safety factor (by a factor of 2) and increase in pressure (by a factor of 2) does not directly relate to a reduction in pressure (by factor of 2) and increase in safety factor (by a factor of 2). In other words half the design pressure and double the safety factor does not result in the same reinforcement as double the design pressure and half the safety factor. In fact, according to the PDV, a crotch plate is required for cases 2 and 3 due to the higher design pressures. A significantly higher yield strength could, however, result in the wrapper/collar not being required at all due to the fact that a higher yield strength impacts on the required minimum wall thickness of the barrel. The 
higher yield strength could result in sufficient excess wall thickness to account for the loss in wall thickness of the barrel as a result of branching. The PDV and diameter ratio of the special in case 4 indicates a wrapper as the proposed reinforcement, but the wrapper is in fact not required as a result of sufficient excess/available barrel thickness.

Table 2.1: Effect of safety factor and design pressure on wrappers and collars.

\begin{tabular}{lrrl} 
PARAMETER & CASE 1 & CASE 2 & UNITS \\
\hline $\begin{array}{l}\text { INPUTS } \\
D\end{array}$ & 610 & 610 & $\mathrm{~mm}$ \\
$d$ & 610 & 610 & $\mathrm{~mm}$ \\
$T_{y}$ & 6 & 6 & $\mathrm{~mm}$ \\
$t_{y}$ & 6 & 6 & $\mathrm{~mm}$ \\
Angle & 50 & 50 & Degrees \\
PDV & 1039 & 1734 & \\
Yield strength & 290 & 290 & $\mathrm{MPa}$ \\
$S F$ & 4.65 & 2 & \\
Allowable yield strength & 62.366 & 145 & $\mathrm{MPa}$ \\
$w$ & 397 & 397 & $\mathrm{~mm}$ \\
$T$ & 6 & 6 & $\mathrm{~mm}$ \\
OUTPUTS & & & \\
Resulting design pressure $P$ & 1.0000 & 1.668 & $\mathrm{MPa}$ \\
\hline
\end{tabular}

Table 2.2: Relationship between safety factor and design pressure for wrappers and collars.

\begin{tabular}{lrrl} 
PARAMETERS & CASE 3 & CASE 4 & \\
\hline$\frac{\text { INPUTS }}{D}$ & 610 & 610 & $\mathrm{~mm}$ \\
$d$ & 610 & 610 & $\mathrm{~mm}$ \\
$T_{y}$ & 6 & 6 & $\mathrm{~mm}$ \\
$t_{y}$ & 6 & 6 & $\mathrm{~mm}$ \\
Angle & 50 & 50 & Degrees \\
PDV & 2078 & 1039 & \\
Yield strength & 290 & 290 & $\mathrm{MPa}$ \\
$S F$ & 1 & 2 & \\
Allowable yield strength & 290 & 145 & $\mathrm{MPa}$ \\
$P$ chosen & 2.0000 & 1.0000 & $\mathrm{MPa}$ \\
OUTPUTS & & & \\
$w$ & 265 & 0 & $\mathrm{~mm}$ \\
$T$ & 4.5 & 0 & $\mathrm{~mm}$ \\
\hline
\end{tabular}


The use of collar plates for diameter ratios higher than 0.7 was investigated to advise on the use of thicker collar plates over wrapper plates. The thickness was increased whilst the reinforcement width remained unchanged. The area replacement calculation in Equation 2.4.6 is identical for wrappers and collars and therefore collars and wrappers with the same width $(w)$ and thickness $(t)$ provide the same reinforcement area. Collar plates are easier and cheaper to manufacture and hence it might prove beneficial to, where possible, use thicker collar plates instead of thinner wrapper plates. Section 3.7 elaborates further on the use of thicker collar plates for specials with diameter ratios larger than 0.7 .

\subsubsection{Crotch Plates: Nomograph Method}

Crotch plates, on the other hand, are designed in accordance with the model published by Swanson et al. (1955) through the use of a nomograph (Figure A.1 in Appendix A). A nomograph is a graphical calculating device which allows the approximate graphical computation of a mathematical function or experimentally obtained values. Nomographs typically have three axes and with two known parameters the third can be obtained. The result is obtained by laying a straightedge across the known values on the scales of the nomograph and reading the unknown value from where it crosses the scale for that value. The nomograph used in M11 is based on design pressure, has a built-in safety factor for surge and takes the effect of welding at the intersection into account. For reducing specials, where the branch diameter is smaller than the barrel diameter, a single plate curved stiffener is used as shown in Figure 2.19.

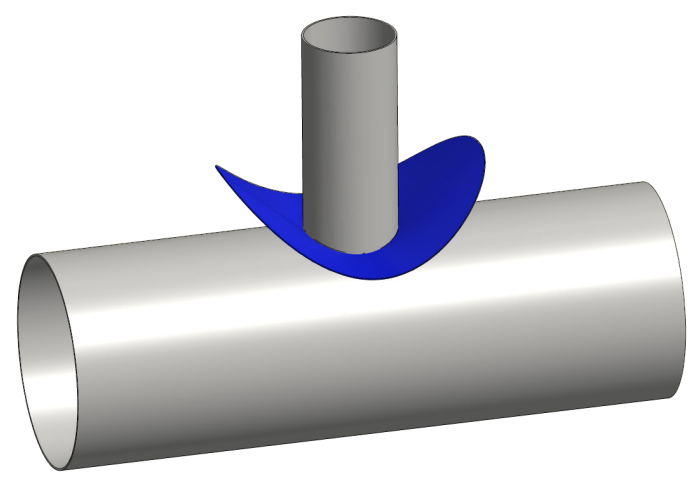

Figure 2.19: Typical single plate crotch plate

The two-plate design (Figure 1.2) is used for equal diameter specials. A third plate (Figure 2.12b) can be added for high internal pressures (typically above $2 \mathrm{MPa}$ ) and large pipe diameters (typically above DN 1500). The nomograph has a built-in safety factor to keep the stresses below the yield strength of steel, but this safety factor is not provided. The design pressure used in 
the nomograph is limited to 1.5 times the working pressure, described further in Chapter 3. The yield strength of the steel used by Swanson et al. (1955) is 30000 psi (approximately $207 \mathrm{MPa}$ ), resulting in an allowable yield strength of approximately $138 \mathrm{MPa}(20000 \mathrm{psi}$ ) under working conditions. The AWWA M11 model therefore limits the maximum stress to an allowable yield strength. The yield strength of modern steel is typically significantly higher than the yield strength used by Swanson et al. (1955). However, the crotch plate design model does not provide for increased yield strengths. An adjusted design pressure and adjusted plate thickness is considered in Section 3.7 to account for the increased yield strength of modern steel. In this study it was found that local yielding occurs even if the design pressure is limited to 1.5 times the working pressure as prescribed by M11. The nomograph is further based on an experimental plate thickness of $25.4 \mathrm{~mm}$ (1 inch) and a deflection angle of 90 degrees. This graph is used to determine the crotch depth $(d)$ of the crotch plate for equal diameter specials. The layout of the wye branch is shown in Figure 2.20. For right angle specials (90 degree tees) the wye-depth $\left(d_{w}\right)$ and base depth $\left(d_{b}\right)$ is equal.
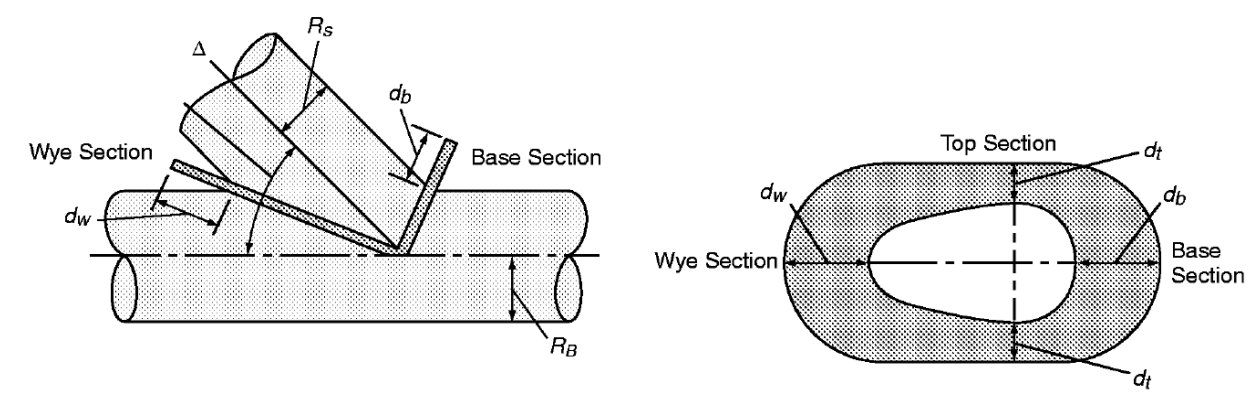

Figure 2.20: Layout and plan of lateral tee (AWWA M11).

The use of a N-factor curve (Figure 2.21) provides two multiplication factors, $N_{w}$ and $N_{b}$, to determine the wye-depth and base-depth for crotch plates on lateral tees respectively. 


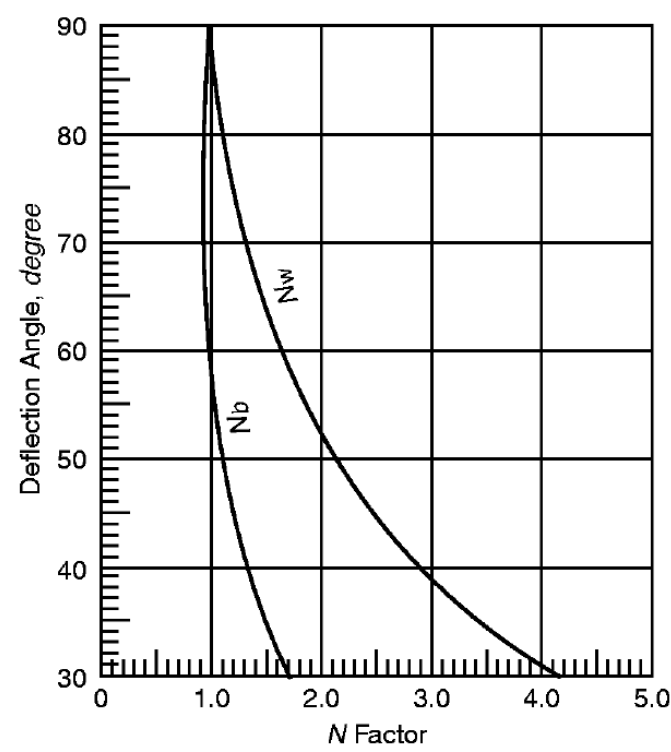

Figure 2.21: N-factor curve for crotch depths (AWWA M11).

For curved single plate stiffeners the wye-depth and base-depth, found on the N-factor curve, must be multiplied by the respective factors found on the Qfactor curve (Figure 2.22). The crotch depth is then calculated as $d^{\prime} w=Q_{w} d_{w}$ and the base depth as $d^{\prime} b=Q_{b} d_{b}$. The radius of the branch pipe and barrel is denoted by $R_{s}$ and $R_{b}$ respectively.

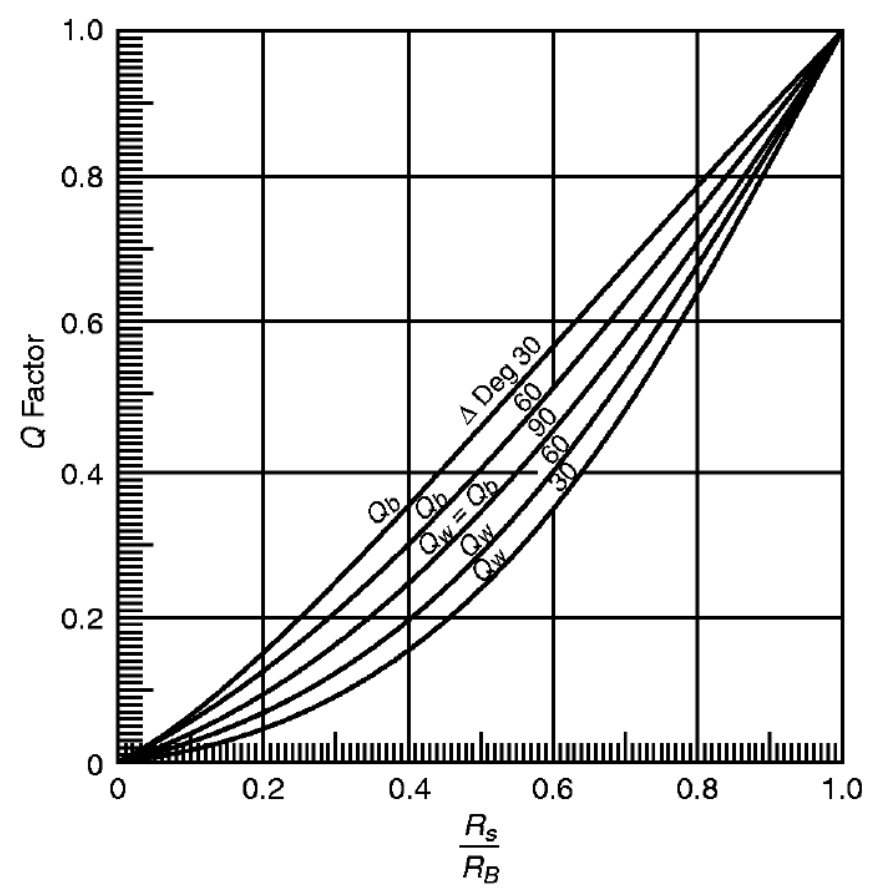

Figure 2.22: Q-factor curve for reducing tees (AWWA M11). 
Equation 2.4.8 is used to scale the crotch depth of the stiffener plate if the wye depth is greater than 30 times the thickness of the experimental plate $(25.4 \mathrm{~mm})$. This is a trial and error approach to find the optimum plate thickness that will result in a crotch depth of less than 30 times the thickness of the experimental plate thickness.

$$
d=d_{1}\left(\frac{t_{1}}{t}\right)^{\left(0.917-\frac{\Delta}{360}\right)}
$$

where

$$
\begin{aligned}
d & =\text { New depth of plate } \\
d_{1} & =\text { Plate thickness determined from Q- and N-factor curves } \\
t_{1} & =\text { Experimental plate thickness }(25.4 \mathrm{~mm}) \\
t & =\text { Estimated optimum plate thickness } \\
\Delta & =\text { Branch pipe angle of deflection (degrees) }
\end{aligned}
$$

To find the top depth ( $d_{t}$ for two plates or $d^{\prime} t$ for single plates) of the reinforcement Swanson et al. (1955) suggested using the graph shown in Figure 2.23. For crotch plates with a curved outer radius, the radius is equal to the sum of the crotch depth (wye-depth or base depth) and the inner radius of the pipe. It is clear from the model provided by Swanson et al. (1955) that the design greatly depends on the accuracy of the various charts as obtained by experimental analyses. 


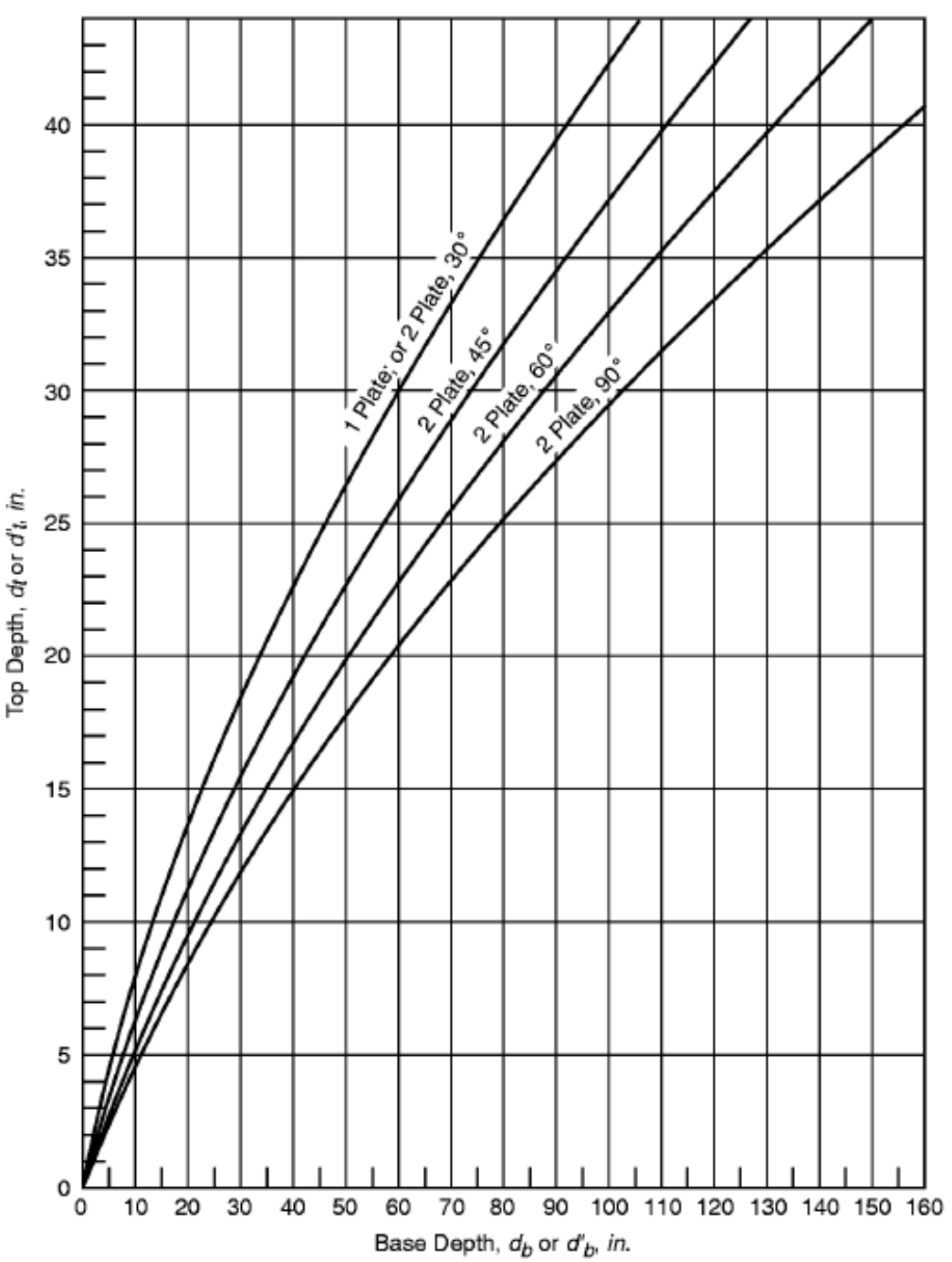

Figure 2.23: Top depth selection chart (AWWA M11).

\subsection{Introduction to FE Methods}

The development of finite element (FE) methods has had a great impact on engineering and more specific, for the purpose of this study, solid mechanics. FE methods are used to build a virtual model and approximate the exact solution of complex systems of partial differential equations. The practical implementation of FE methods is often referred to as FE analysis (FEA) and a wide range of software is available to solve and graphically present the results when solving, amongst others, structural problems. FE methods and the process of solving a problem by $\mathrm{FE}$ analysis is described in more detail in Chapter 3. Although the history and development of FE software is beyond the scope of this study, some clarity is indeed justified and discussed briefly 
in Chapter 3. Important for this study is the difference between standalone FE Analysis solvers and CAD-based solvers. Patran (using Nastran as solver) and Ansys are two popular standalone packages. Common CAD-based solvers include Autodesk Inventor and SolidWorks. Newer fourth-generation software allow the user to perform a more reasonable amount of editing on geometry imported from CAD packages. Altair Hyperworks has proven, for this study, to be a stable and useful fourth-generation FE package with an integrated pre-processor, solver and post-processor.

With standalone packages the 3D model is designed in some CAD software and imported into the FE analysis package. This leads to increased complexity and requires additional software licenses and expertise. Standalone packages, however, generally provide the capability to solve more complex and a wider variety of problems using more advanced solvers (Roith et al., 2007). CAD-based solvers on the other hand allow better time management with the model not having to be imported and modified. These CAD-integrated packages (such as Inventor) often provide a financial benefit through an all-in-one solution. The advantage of working in only one graphical interface provides a streamlined and productive design process (Inventor). The disadvantage is that the designer has limited control over the simulation and most CAD-based solvers does not allow the user to select a wide variety of elements, perform mesh refinements or edit solver parameters. 


\section{Chapter 3}

\section{Finite Element Analysis of Cylinder-Cylinder Intersections}

\subsection{Numerical Modelling}

Results obtained from FE analyses are not exact solutions to real world problems. Rather, FE analyses are numerical solutions aimed at approximating the exact solution of a representative mathematical model in the form of a partial differential equation. It is therefore an approximation by piecewise interpolation of some field quantity, such as the displacement field in stress analysis. Cook et al. (2002) points out that numerical analysis software are based on theory and approximations. The validity range of such software is therefore of great importance and designers should take great care in the modelling of finite element problems. Finite element formulations are contained in an easy to use form in general purpose FE analysis programs and therefore it is possible to use these software with little knowledge of the method of analysis. Sadly, most FE analysis results are therefore not reliable and the consequences could be disastrous. Cook et al. (2002) recommends a systematic approach for solving practical problems with FE analysis. The approach recommended by Cook et al. (2002) is followed in this study through classification of the problem, mathematical simplification of the problem, performing preliminary analyses of the problem as a basis for setting up the FE analysis problem and subsequently defining the FE analysis inputs and assumptions. Thereafter the problem is modelled with consideration to linear and nonlinear behaviour, midsurfacing, contact between surfaces and mesh refinement. This is followed by numerical analysis of the problem and post-processing of results.

\subsection{Problem Classification}

It is important that the nature and extent of a problem be well understood prior to undertaking a FE analysis. In this study the problem considered is 
the simulation of failure loads of reinforced steel specials in order to determine the resulting safety factor under internal pressure loads. The user must define whether the problem is of a linear or nonlinear nature as this will determine the software required to perform the analysis. The type of nonlinearity must also be defined, for example material or geometrical nonlinearity. Material which follows Hooke's law is said to be of a linear nature. The maximum stress at which Hook's law is satisfied is termed the yield strength of the material. Above the yield point the material moves into the plastic region where the relationship between stress and strain is nonlinear i.e. material nonlinearity. This also occurs where material properties change with applied loads. Geometric nonlinearity is when large deformations and/or rotations occur such that the applied loads may change direction, or where the equilibrium equations from which the nodal displacements and rotations are solved must be written in the deformed state instead of the undeformed state (Cook et al., 2002). A third type of nonlinearity in structural mechanics occurs where parts make or break contact i.e. where the stiffness of the structure changes, based on the contact condition which depends on the applied load.

The AWWA M11 design code limits the design stress (maximum calculated stress) of the steel to the yield stress of the steel for a safety factor of 1 and therefore the steel is deemed to have failed should the maximum stress in the steel reaches the material's yield stress. For higher safety factors the design stress is reduced. Thus, a safety factor of 2 corresponds to an allowable stress of half the yield stress. This is considered to be a conservative approach as the plastic behaviour of the steel, especially ductile steel, is not taken into account. In this study the steel is allowed to yield locally (material nonlinearity) with the assumption that the special will not necessarily fail due to local stresses above the yield strength. This is under the assumption that only ductile failure is considered and brittle failure or failure from defects are not considered. The purpose of the nonlinear analysis is to determine how much load (internal pressure) the special can support before global ductile failure occurs. With the assumption of elastic perfectly plastic behaviour the safety factor is calculated using the design pressure and internal pressure at which the special fail based on numerical analysis. Ductile failure for an EPP case is regarded as the load case resulting in non-convergence of the nonlinear problem. Hence, the plastic region of the stress-strain characteristics of steel becomes important and nonlinear problems are to be solved. The displacements are expected to be small in comparison with the size of the specials. Large displacements are typically expected with rubber structures and metal forming processes. Large rotations are expected with, amongst others, springs and cables. The contact between the wrapper/collar and barrel in this study is not broken. The problems in this study are thus not considered to be of a geometrical or contact nonlinear nature and only material nonlinearity was considered. 


\subsection{Mathematical Model}

Prior to performing a FE analysis it is important to simplify a problem as much as possible. Only important features should be modelled and constraints, supports, loads and boundary conditions should be simplified as far as possible. Geometric irregularities are often ignored to simplify the problem. The mathematical model is then based on the simplified problem and the analysis theory to be applied to it. Material properties must be determined and idealized, if possible, in order to simplify the analysis. FE analyses pertain only to the theoretical mathematical model and therefore it is important to define the mathematical problem as accurately as possible. The results of FE analyses may very well suggest and demand refinements to the geometry, loads and boundary conditions.

In this study a number of features were simplified to better present the mathematical model. The effect of the welds between the reinforcement, branch and barrel was ignored. The heat affected zone around the welding is a complex problem as no two welds in practice are exactly the same. Failure as a result of welding defects were not considered in this study. Rather, it is suggested that the design safety factor should be adjusted to allow for welding effects such as heat affected zones. In this study a safety factor of two was used with the assumption that the correct welding procedures are followed and welds are thoroughly inspected and tested for defects. Modern technologies such as radiographic testing can to a large extent, although probably not completely, limit such defects. This assumption is supported by practical experience with reinforced steel specials installed in bulk water pipelines. Hydrostatic tests were conducted (see Chapter 4) to compare typical material failure in practice with FE analyses results. Figure 2.7 on page 10 shows the overlapping of elements at the branch/barrel intersection. The effect of overlapping elements between the branch and barrel was ignored as this is inevitable with a FE analysis using shell elements and considered to have minimal impact on the results.

\subsection{Preliminary Analysis}

With any FE analysis it is important to obtain a preliminary solution to the problem. This can also be done using simple calculations, practical experiments, previous experience or design codes. When FE analyses are performed prior to obtaining preliminary solutions the user generally tends to look for data from physical testing that supports $\mathrm{FE}$ analysis results rather than using FE analysis to support the physical data. By starting with experimental results the user is forced to thoroughly review the FE model when the FE analysis results are different to experimental results. Preliminary analyses help the user to narrow a problem down and hence minimise the number of model revi- 
sions required. However, it is worth pointing out that FE analyses often have an impact on physical testing and vice versa. This study developed from past experience with steel tee reinforcing designs that were based on experimentally developed formulas. Previous experience with hydrostatic testing and installations of these tees were used as a benchmark in the interpretation of FE analysis results. This lead to better mathematical modelling and interpretation of the FE model. The FE analysis results must finally be validated to conclude on the accuracy of assumptions, simplifications and modelling techniques. In this study assumptions, simplifications and modelling techniques used were validated with a physical experiment. The AWWA M11 model is used as a sense check in interpretation of the simulated results. It is assumed that the nature of the AWWA M11 design model lends itself to being conservative as it is not intended to be an exact analysis tool, but rather a practical tool for the design of reinforced specials that would not fail. The experimentally obtained M11 model is therefore not used to validate the FE analyses performed in this study. Rather, a physical experiment is done in this study to validate a typical $\mathrm{FE}$ analysis performed in this study.

\subsection{Finite Element Analysis}

\subsubsection{Preprocessing}

Three sources of errors are widely recognised in FE analysis: modelling errors, discretization errors and numerical errors. Numerical errors result from numbers of finite precision used in the manipulation and representation of data. These errors are controlled automatically by software. Modelling errors occur due to errors in the geometric modelling of a simulated structure (in the case of structural mechanics). These errors can be reduced by improving and simplifying the model geometry. The number and type of elements used in a structure largely determine the discretization error. It is therefore critical to select the best possible type of element, element size and proper distribution thereof to restrict the discretization error to a minimum.

Many finite element studies have been conducted on cylinder/cylinder intersections using both $2 \mathrm{D}$ and $3 \mathrm{D}$ elements. It was noted by Attwater et al. (1994) that the choice between a higher density of lower-order elements and a less dense system of higher-order elements is not straightforward. In general the selection and placements of elements are critical for an accurate analysis. The complexity of surface interactions eliminates the use of certain elements. The tees modelled in this study are thin walled specials, where the wall thickness of the pipes are typically one hundredth of the diameter of the pipe. First order bilinear quadrilateral (Q4) shell elements with six degrees of freedom per node were used in this study. The shell elements in Optistruct have built- 
in drilling degree of freedom. Although first order bilinear quadrilateral shell elements cannot exhibit pure bending, the pipes and reinforcing plates are classified as thin-walled members and the effect of bending is therefore considered to be negligible when a large number of small elements are used in thin plate bending. Cook et al. (2002) points out that the addition of drilling DOF can enable elements having only corner nodes to provide acceptable performance compared to elements having both corner and side nodes with less degrees of freedom. As a result first order bilinear quadrilateral shell elements are considered to be sufficient for modelling the specials in this study and therefore second order quadrilateral elements having second order polynomial functions are not preferred, largely due to increased analysis effort.

For reinforcing crotch plates the thickness is also small compared to the width and length of these plates and hence crotch plates were also modelled with Q4 shell elements. It was therefore decided that second order quadrilateral elements having second order polynomial shape functions are not required. Three dimensional elements supply more degrees of freedom than are required to model the shells of the reinforcing tees considered in this study and thus were not considered.

A number of assumptions are typically made for numerical analyses. The following assumptions, as pointed out by Xu et al. (1999), can be made for steel tees where the resulting stresses are within the proportional limit of the material:

- The material is homogeneous and isotropic.

- The resulting stresses obey Hooke's law.

- Self-weight is neglected.

- For unreinforced tees there are no reinforcing pads/fillets at the junction (branch/barrel intersection).

- The barrel and branch pipe ends are flanged and hydrostatically loaded.

The assumptions made by the user could greatly affect the results of the simulation. Due cognisance of the physical problem is required to make the right assumptions for each model. The following general assumptions were made in this study for all numerical models, except for the test piece or where otherwise noted:

- The material is homogeneous and isotropic.

- The material is elastic perfectly plastic and hence strain hardening does not occur. This is considered to be a conservative design approach. However, failure is not easy to predict/simulate in the plastic zone and 
the Von Mises stress criteria is not valid for stress values above the yield strength. An alternative triaxial stress criteria would therefore have to be considered. This approach is already a big improvement over a purely linear elastic analysis and an alternative to linear elastic analysis.

- The material has a tensile (Young's) modulus of $200 \mathrm{GPa}$.

- The Poisson ratio is taken as 0.3.

- The resulting stresses are not necessarily within the proportional limit and therefore yielding could occur.

- The yield strength of API 5L Grade X42 pipe material often used in bulk water steel pipelines and in this study is $290 \mathrm{MPa}$ (API Specification 5L). The purpose of this study is to see how the AWWA M11 code (based on a yield strength of $207 \mathrm{MPa}$ compare against newer steels and detailed FE analyses.

- The effect of the welded area at the barrel/branch/reinforcing plate intersection is negligible.

- Local material yielding is not necessarily a measure of ductile material failure. Rather, a special is considered to have failed when the nonlinear analysis does not converge i.e. the material is yielded to such an extent that no further local yielding occurs. This assumption is made under the condition that the necessary care is taken in the numerical analysis to ensure the load at non-convergence is similar for, amongst others, different time steps and number of iterations used.

Another important factor is the careful selection and accurate placement of boundary conditions. Boundary conditions are the application of forces and/or constraints to a model. This is a fundamental part of any FE analysis and can greatly affect the results of any simulation. Minor changes in boundary conditions can have a major effect on computed results. Figure 3.1 shows a typical wrapper reinforced tee with slip-on flanges welded to the ends of the tee and blank flanges bolted (fasteners not shown) thereto in order to allow for hydrostatic testing. The hydrostatically loaded specials are considered to be standing on a slab of concrete and not constrained to move and rotate as it deforms. The boundary conditions are thus only to remove the rigid body modes from the structure, but should not impose additional loads or stresses on the structure. The boundary conditions used in this study were chosen to represent this setup. 


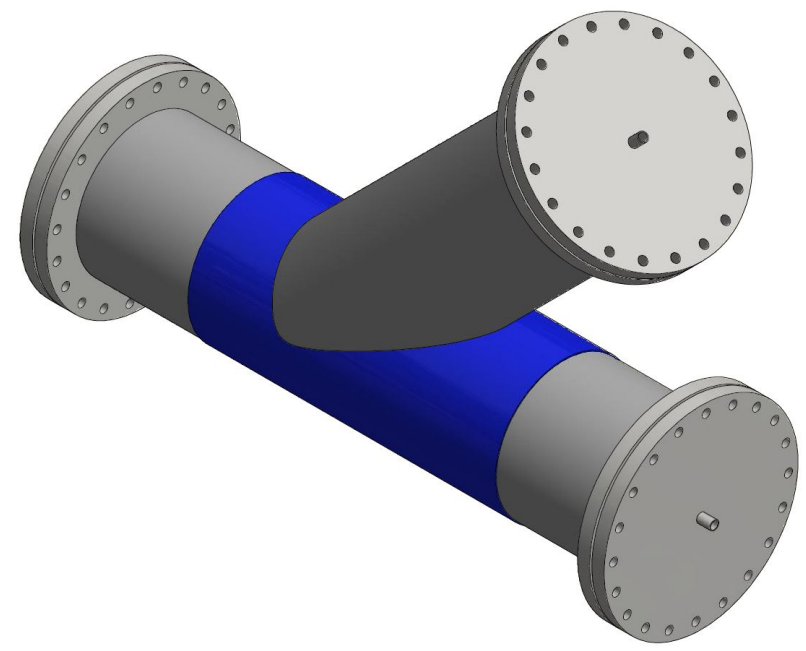

Figure 3.1: Flanges on typical wrapper reinforced tee.

The ends of the barrel and branch are considered to be infinitely rigid in the radial direction when compared to the rigidity of the rest of the pipe due to the thick flanges on the pipe ends. Rigid (RBE2) elements were used to constrain all nodes on the open ends of the pipes to an imaginary (independent) node centered between the outer edge (dependent) nodes. Figure 3.2 shows a typical RBE2 element that connects all edge nodes on a pipe end. The rigid element prevents relative displacement and rotation between selected nodes. Displacements in the axial direction occur due to the longitudinal stresses in the pipe walls which in turn result from pressure forces acting on the blank flanges bolted to the pipe ends. A force equal to the pressure forces acting on each pipe end is then applied to the respective center node and this force is distributed equally to the dependent nodes of the RBE2 element on the edge of the relative pipe end.

The barrel and branch end pressure forces were calculated as follows:

$$
F_{p}=P_{i} A
$$

where

$F_{p}=$ Pressure force acting on pipe end

$P_{i}=$ Internal pressure in the relevant pipe

$A=$ Internal area of the relative pipe end 


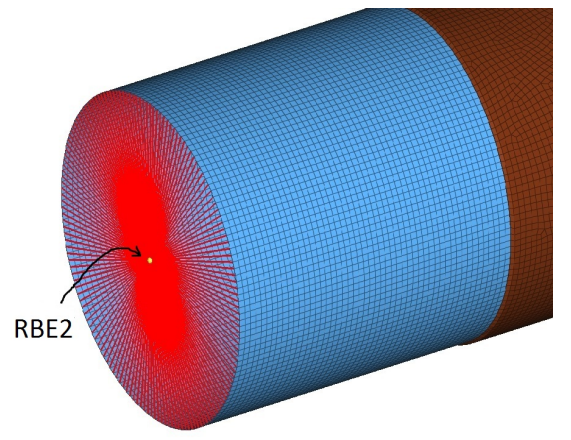

Figure 3.2: RBE2 element (shown in red) connecting the center node to outer edge nodes.

The specials were first modelled with RBE2 elements on the branch and barrel ends to only allow displacements in the axial direction of the corresponding pipe. Figure 3.3a shows a typical stress distribution for these boundary conditions. A review of the boundary conditions pointed out that these boundary conditions do not represent actual constraints and forces for tees under hydrostatic loading as it does not allow relative displacement and rotation between the three pipe ends. The boundary conditions were then changed with one end of the barrel fully constrained (to prevent rigid body modes i.e. singular modified stiffness matrices) and the other end constrained only in the Y-direction, as shown in Figure 3.3c. Results shown in red are high stress and blue low stresses. With the material assumed to be elastic perfectly plastic all elements in red have yielded. Visual observations with the assistance of high definition camera recordings, however, showed significantly larger deformations. The boundary conditions were reviewed once more and it was found that these boundary conditions also over-constrain the model. Only the RBE2 element on one end of the barrel was then fully constrained and all other nodes left unconstrained. Strain measurements, described in Chapter 4, confirmed that these boundary conditions best represent the relative displacement and rotation between the pipe ends of a hydrostatically loaded specials. Also, high stresses do not exist at the point where the boundary conditions are attached. Figure 3.3d shows the stress distribution with only one end of the barrel constrained. The internal pressure, at failure of the three specials, is shown in brackets for the three boundary condition cases. 


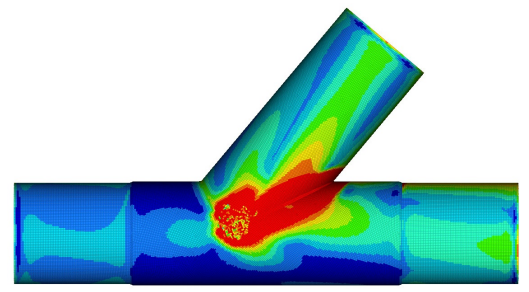

(a) RBE2 elements on edge nodes of tee ends constrained to allow movement only in the axial direction $(2.6 \mathrm{MPa})$.

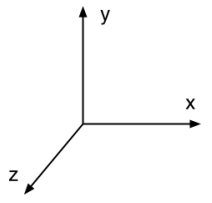

(b) Global coordinate system.

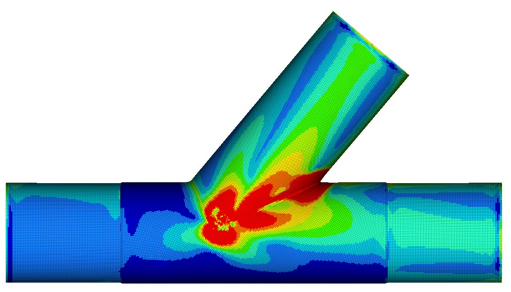

(c) One end of barrel fully constrained and other end constrained against movement in the Y-direction (2.4 MPa).

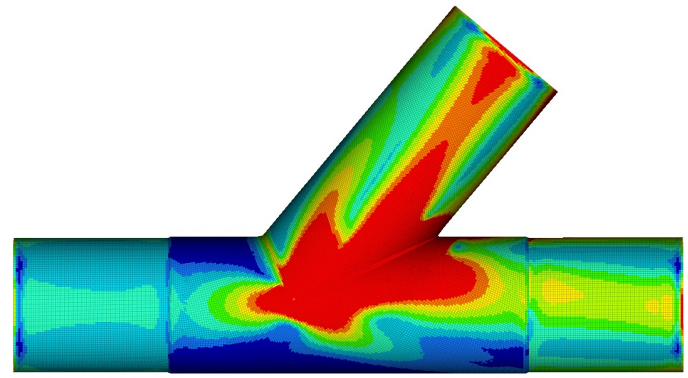

(d) One end of barrel fully constrained (3.6 MPa).

Figure 3.3: Effect of different boundary conditions, with red a high (yield strength) and blue a low stress.

\subsubsection{Numerical Analysis}

Finite elements can be formulated by a number of well-known techniques. These techniques include, amongst others, direct physical argument, a virtual work argument, variational arguments applied to functionals such as potential energy and the weighted residual method. These techniques are used to develop formulas for the characteristic matrix of a finite element and which describes element behaviour. In structural mechanics the characteristic matrix is also called the element stiffness matrix. These techniques have been widely accepted and the theory behind it is beyond the scope of this study. However, for the purpose of this study it is important to point out that a FE user must be well informed on factors such as number of degrees of freedom and how the FE formulation for a given problem is obtained. Cook et al. (2002) points out that in order to produce reliable results it is important that the user understands the problem, know how to model it, understands element behaviour and limitations, software limitations and how to check for errors in results. Cook et al. (2002) further points out that an error caused by misunderstanding or oversight is not correctable by mesh refinement or by use of a more powerful computer. This is an important observation and is supported by the impact that boundary conditions, discussed in Section 3.5.1, have on results. 


\subsection{Modelling of Steel Specials}

\subsubsection{Linear and non-linear behaviour}

Simple linear static FE analyses were firstly done to determine whether resulting stresses typically reach the yield stress of the material for loads that comply with the M11 design code. These relatively simple analyses were done using Inventor's FE solver and later with Optistruct. Preliminary results of linear static analyses have shown that local yielding does indeed occur for hydrostatic loads that are well within the allowable range given by the M11 code. Once the stress in the material reaches the yield point thereof the strain is no longer a linear function of stress. With increasing loads, strains start to increase exponentially, according to the stress-strain curve of the material, until failure thereof. Figure 3.4 shows the result of a typical linear stress analysis generated in Inventor Professional. This figure of a DN 600 tee shows a colour shading representation of the stresses in the pipe shell where yellow is a high and blue a low stress area. The shell is subjected to an internal pressure of 1.6 MPa. It can be seen that at the intersection the Von Mises stresses are higher than the yield strength with yellow indicating yield strength and blue lower stresses. In this case the highest stresses are in excess of the yield strength of the steel.

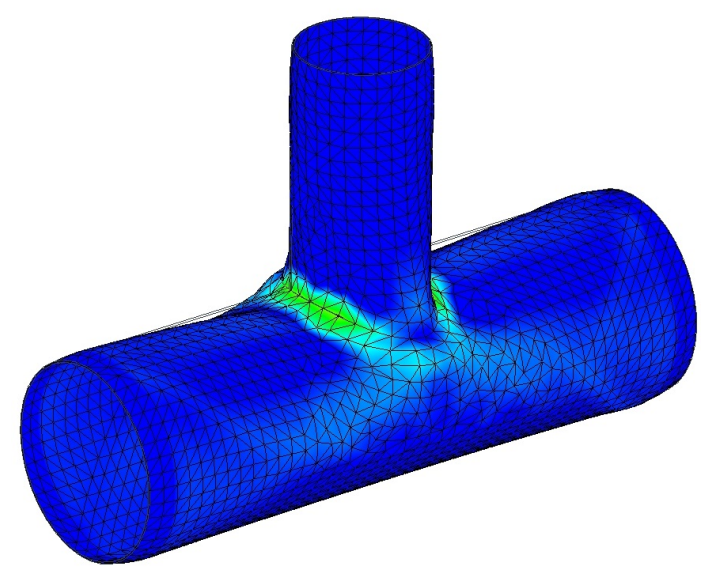

Figure 3.4: Typical Autodesk Inventor stress analysis.

These local high stress regions are typically a result of the complex geometric interactions between surfaces and the inability of elements (described in Section 3.5.1) to accurately represent the true interaction between surfaces. Mesh refinement and geometric simplifications such as the elimination of welds can greatly reduce, albeit not eliminate, modelling errors. To account for local stresses above the yield strength of the material and the complex interactions of surfaces a nonlinear solver was required. When problems are of a nonlinear nature the stiffness and possibly loads become functions of displacement or deformation (Cook et al., 2002). The structural equation is typically defined as: 


$$
[K]\{D\}=\{R\}
$$

where

$$
\begin{aligned}
{[K] } & =\text { Stiffness matrix } \\
\{R\} & =\text { Load vector } \\
\{D\} & =\text { Displacement vector }
\end{aligned}
$$

The displacement vector cannot be solved immediately such as with a linear analysis as information needed to construct the stiffness matrix and the load vector is not known in advance. An iterative process is thus required to obtain the displacement vector and its associated stiffness matrix and load vector such that the equation is in equilibrium. The geometry, boundary conditions and material properties are changed after each load increment and the stiffness matrix updated accordingly. The principle of superposition thus does not apply for nonlinear problems and therefore each load case requires a separate analysis of the structural equation. This equation can be solved by a rather large number of different methods such as the Newton-Raphson method used by Optistruct for nonlinear static analyses as in this study. Iterations are performed for each load case to determine equilibrium of the structural equation at that load case. Equilibrium is achieved when the predefined convergence criteria is satisfied. According to Cook et al. (2002) convergence can be tested using a number of methods such as a small force imbalance compared to the total applied force or a small displacement increment compared to the initial displacement increment. Optistruct uses a force imbalance $\left(\left\{e_{R}\right\}\right)$ defined as follows:

$$
\left\{e_{R}\right\}=\{R\}-[K]\{D\}
$$

Convergence is achieved when the force imbalance is smaller than the predefined criteria/error margin. Non-convergence occurs when, for some load factor, a solution to the set of algebraic equations approximating the exact solution can no longer be found i.e. equilibrium of the structural equation is not achieved.

Inventor's built-in solver is not capable of nonlinear analyses and it does not allow the user much options with element selection, mesh refinement or defining boundary conditions. Although the linear analysis shows the high stress and large deformation regions accurately, it does not provide an accurate representation of the magnitude of stresses and strains. Subsequently it was decided to use MSC SimXpert. However, SimXpert was found to be insufficient in dealing with the complex midsurfacing of the various surfaces and as a result MSC Patran was used. It was found that both SimXpert and Patran does not allow for easy midsurfacing of the solid models exported from 
CAD software packages such as Autodesk Inventor. Neither does it provide for cleaning up the complex geometry of reinforced cylinder/cylinder intersections. Limited options were available due to license options available. As a result it was decided to instead switch to the Altair Hyperworks suite of products, which includes a pre-processor (Hypermesh), solver (Optistruct) and post-processor (Hyperview). Hypermesh provide advanced geometry and meshing capabilities to deal with complex surface interactions. Although it is not a computer aided design tool it does offer a wide range of modelling tools to simplify surfaces, adjust geometry and greatly simplifies mesh generation and modification. Optistruct is a structural analysis solver for linear and nonlinear problems under static and dynamic loadings. Hyperview is a complete post-processing and visualization environment which enables the user to visualize data interactively and provides animation and plotting features. The integration between the various software is also very smooth. For this study the Hyperworks suite of products thus proved to be the most suitable of the available packages.

\subsubsection{Midsurfacing}

In most practical applications specials are modelled as solid models. In this study all specials were modelled from scratch and therefore shell models were used to avoid the process of midsurfacing. However, for practical applications it is recommended that midsurfacing be done on the available solid models instead of recreating each special as a shell model. When extracting mid-surfaces in Hypermesh or any other pre-processor, however, it is inevitable that some material will be lost. Figure 3.5 shows the surface losses due to midsurfacing.

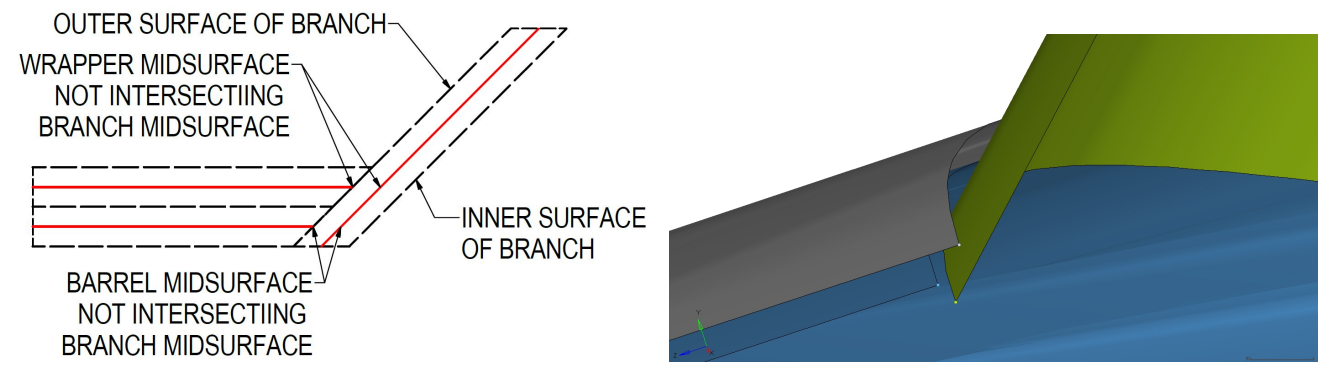

Figure 3.5: Surface losses due to midsurfacing.

In order for the surfaces to intersect each other the midsurfaces must be extended. Rebuilding these surfaces to intersect each other correctly is challenging and time consuming. To avoid the extraction of midsurfaces and hence the extension thereof to each other, the tees were instead modelled in Inventor as midsurface shells. Figure 3.6 shows a typical shell model of a wrapper reinforced tee. 


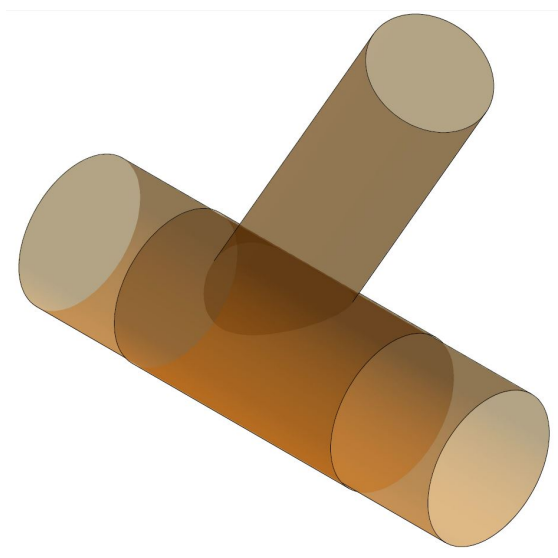

Figure 3.6: Shell model of wrapper reinforced tee.

The shell models were then imported into Hypermesh. The red lines in Figure 3.7 shows that the barrel, branch and reinforcing plate surfaces are not intersected after importing the shell model from Autodesk Inventor to Hypermesh. The surfaces were then intersected in Hypermesh and excess surfaces deleted to clear the internal volume of the pipes as can be seen in Figure 3.8. The free edges are indicated by red lines and edges shared by two or more surfaces are shown in green and yellow. Where surfaces intersect each other it is important that there is a common intersecting edge between the surfaces to ensure that element nodes are equivalenced.

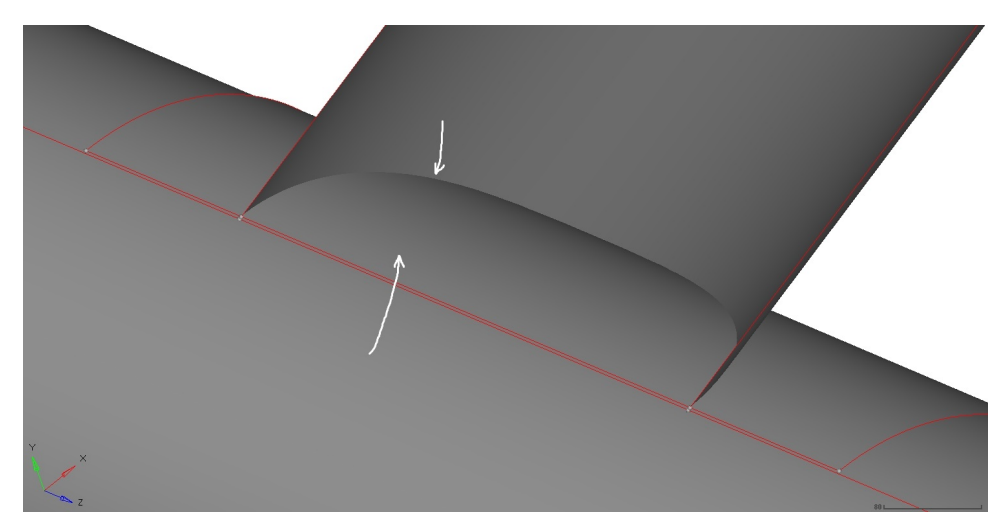

Figure 3.7: Barrel, branch and reinforcing shell surfaces not intersected.

The intersected midsurfaces were then meshed in Hypermesh. Each element was offset by half the thickness thereof to represent the internal and external (top and bottom) surfaces of the pipes and reinforcing plates. A typical meshed midsurface is shown in Figure 3.9. Some of the elements are masked for clarity. 


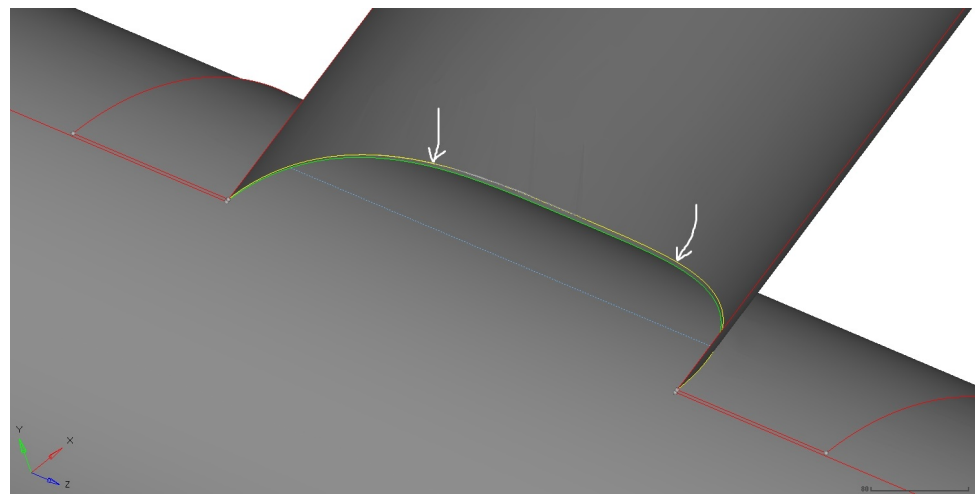

Figure 3.8: Surfaces intersected and excess internal surfaces removed.

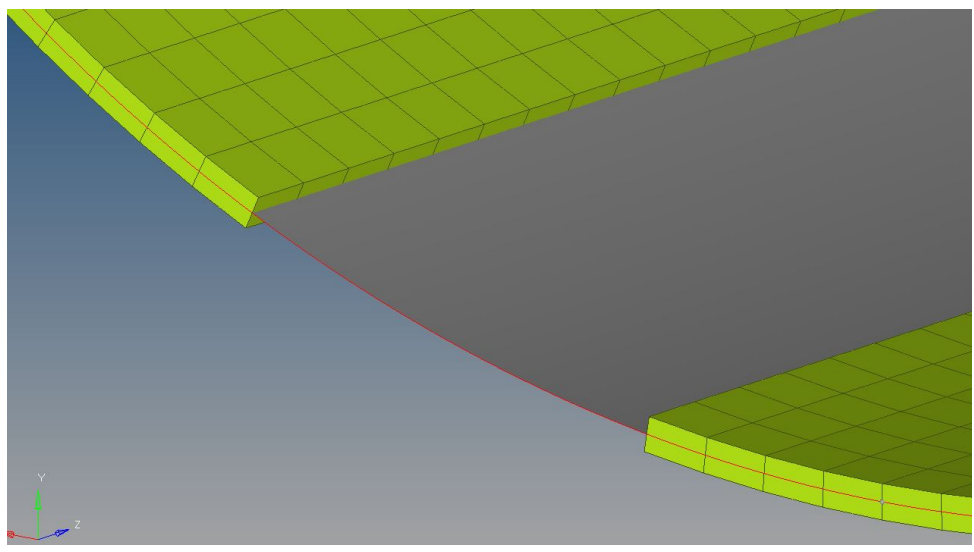

Figure 3.9: Midsurface meshed with offset elements.

\subsubsection{Mesh refinement and overlap}

The width of the narrow surface between the branch-barrel and branch-wrapper intersections is determined by the thickness of the barrel and wrapper. With thin-walled vessels this region is typically very narrow and hence mesh refinement is required in this region to prevent, amongst others, skew elements and to better represent the curved surface. Figure 3.10 shows a typical element distribution of a collar reinforced tee with some elements on the collar hidden for clarity. It can be seen that the elements on the barrel follow the curved edge of the collar. The white elements show the mesh refinement on the narrow surface between the branch-barrel and branch-wrapper intersections. 


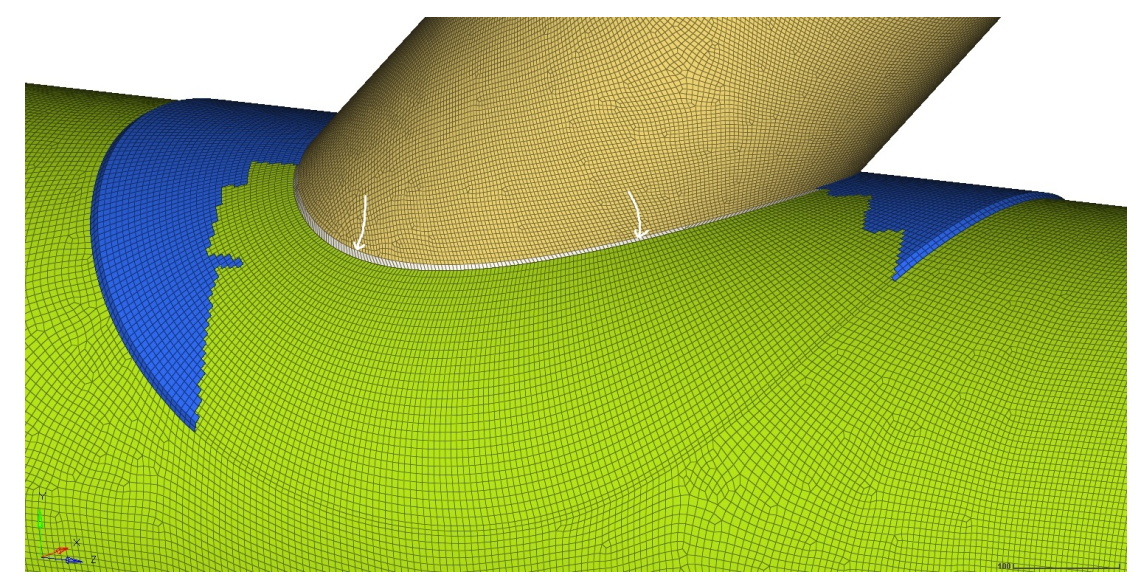

Figure 3.10: Mesh refinement on collar reinforced tee.

In general, increasing the number of nodes improves the accuracy of results as indicated by Figure 3.11, but it comes at an increased solution time and cost. Crotch plate reinforced specials were first modelled with larger elements. After reasonable results were obtained the meshing was refined to test for convergence. Models were deemed to have converged where the failure load was within one decimal place of the coarser mesh. Typical mesh refinement on a crotch plate reinforced special is shown in Figure 3.12. According to the numerical analyses the specials failed under hydrostatic pressures of $5.56 \mathrm{MPa}$ (Fig. 3.12a), 5.55 MPa (Fig. 3.12b) and 5.56 MPa (Fig. 3.12c) respectively. It can be seen that no further convergence is achieved with these mesh refinements and it can be concluded that the coarser mesh in Figure 3.12 seems to be sufficient for convergence. An even coarser mesh could possibly be sufficient for convergence.

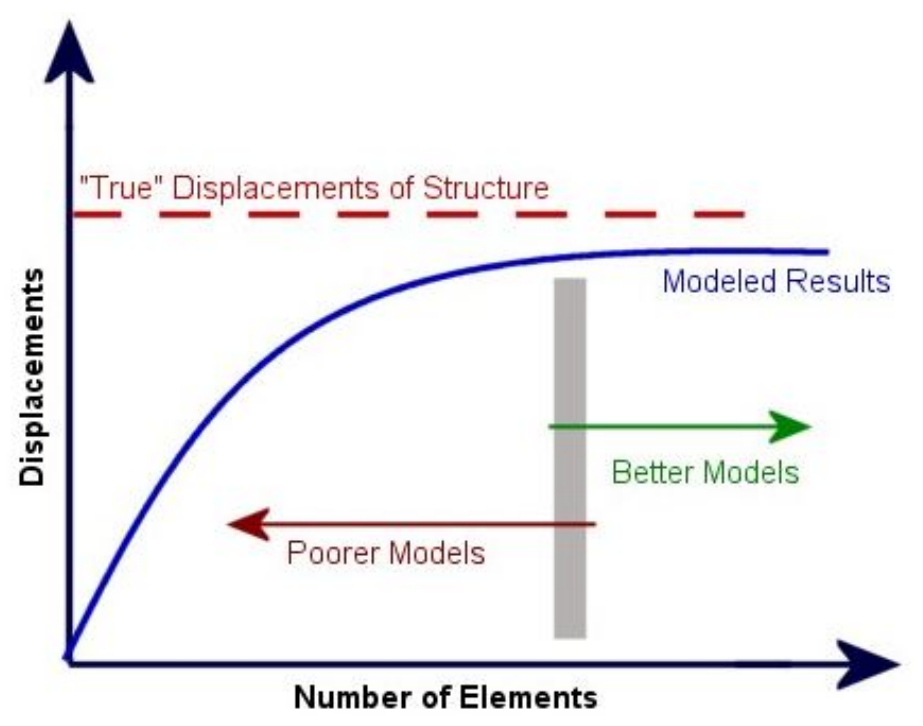

Figure 3.11: Convergence of numerical results (Visual Analysis). 


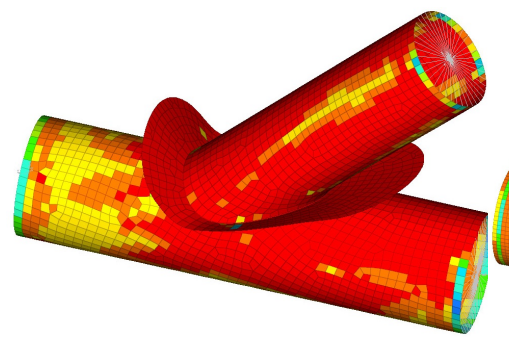

(a) 4025 elements

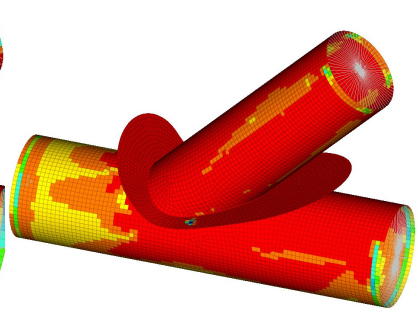

(b) 10656 elements

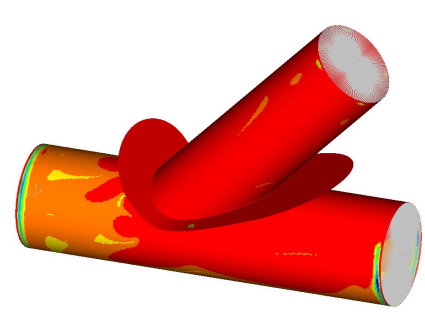

(c) 145215 elements

Figure 3.12: Mesh refinement on croth plate reinforced special, with red a high (above yield strength) and blue a low stress.

Likewise, mesh refinement was done on a DN 600 wrapper reinforced lateral tee, shown in Figure 3.13, to indicate the convergence of results between a typical coarser and finer mesh. The coarse mesh consists of approximately 24500 Q4 elements with an average size of $40 \mathrm{~mm}$ and the finer mesh consists of approximately 110000 Q4 elements with an average size of $10 \mathrm{~mm}$. It turns out that both models produced more or less the same results, with the failure load indicated in brackets for each case. As with the crotch plate reinforced tees described above, further mesh refinement does not seem to improve convergence significantly. Collar reinforced specials have proven the same. The finer mesh required a substantial amount of additional computational effort and it is therefore recommended to always start with a coarse mesh and approach convergence incrementally. For large diameter steel pipe specials relatively coarse meshes turn out to be adequate, at least in areas further away from high stress zones. In order to save on computation effort and time, mesh refinement should be limited to the acute corner and intersection region of barrel, branch and reinforcing plate(s). Convergence was achieved in this study with a relatively coarse mesh with mesh refinement limited to the acute corner and intersection region. 


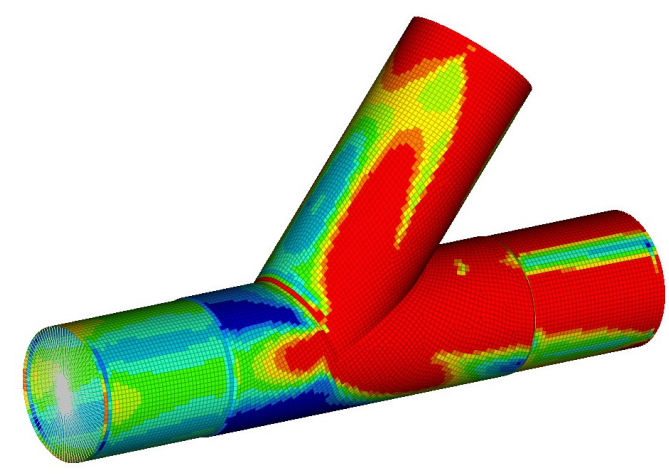

(a) Coarse mesh (3.67 MPa).

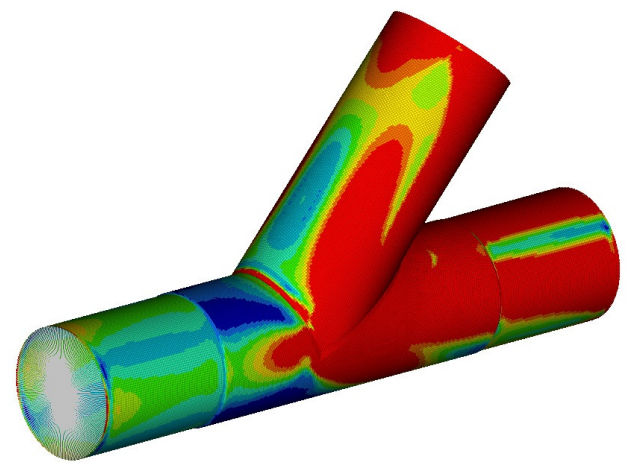

(b) Refined mesh (3.63 MPa).

Figure 3.13: Mesh refinement on equal diameter wrapper reinforced lateral tee.

Another common modelling error is introduced by elements of meshed surfaces overlapping each other due to the thickness of elements. The thickness of an element impacts the stiffness matrix and therefore has an effect on FE analyses. This is, however, inevitable with shell elements and intersecting surfaces, as can be seen in Figure 3.14 and Figure 3.15. The area of overlapping is very small compared to each surface and therefore considered not to have significant impacts on the results.

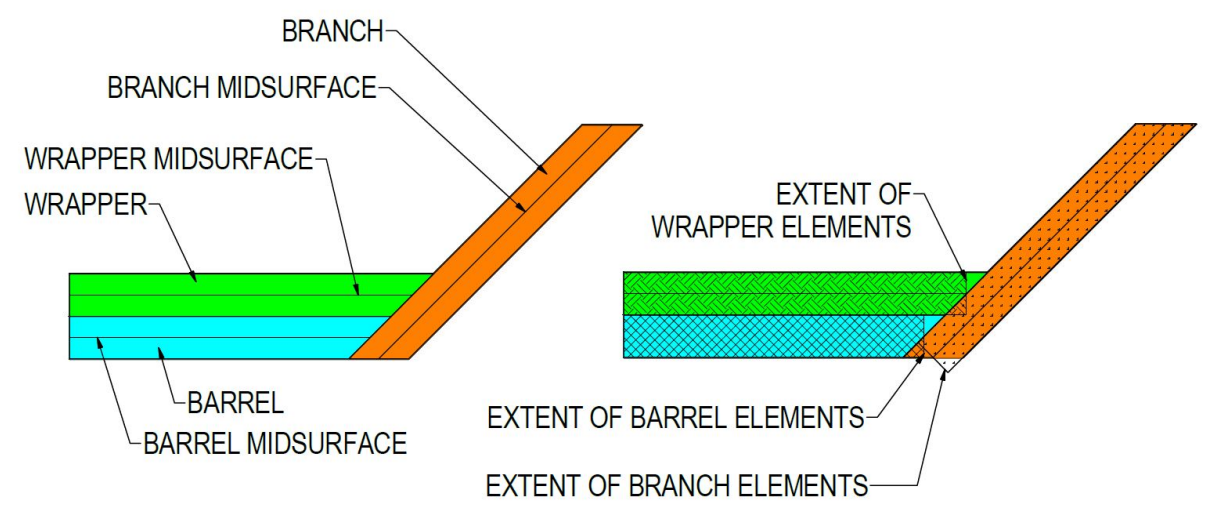

Figure 3.14: FE approximation of surface interactions.

\subsubsection{Contacts}

In the manufacturing of reinforced cylinder-cylinder intersections the branch is welded to the barrel before the reinforcing plate is welded onto the tee, as shown in Figure 3.16a. The reinforcing plate is then welded to the barrel and branch along the edges of the plate as shown in Figure 3.16b. The welded 


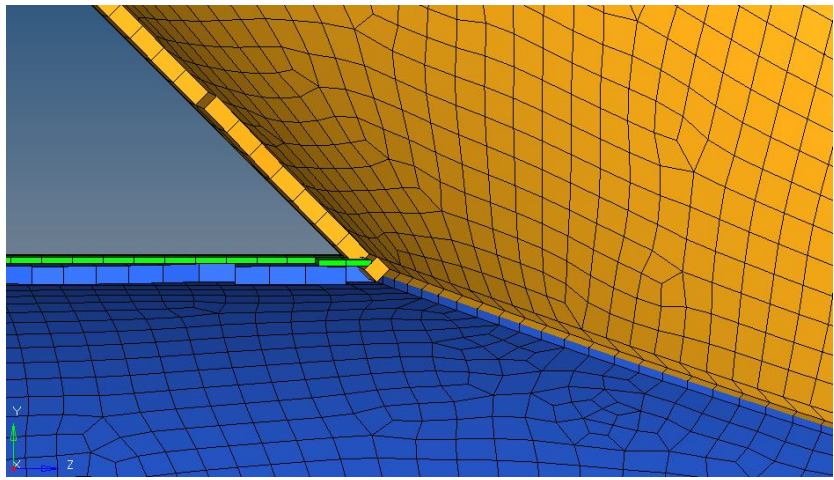

Figure 3.15: Element overlap at branch (orange), barrel (blue) and reinforcing plate (green) intersection.

areas are indicated in yellow. The wrapper is indicated in blue and the barrel in green. In order to mimic the weld on the outer edges of the reinforcing plate and to create an intersection with the barrel the reinforcing plate was extended to the barrel plate as shown in Figure 3.17. Some reinforcing plate elements are masked for clarity.

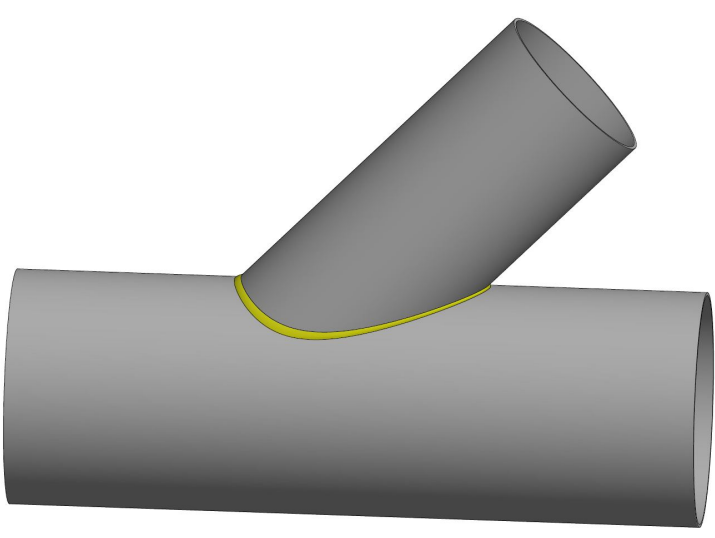

(a) Welding on unreinforced tee.

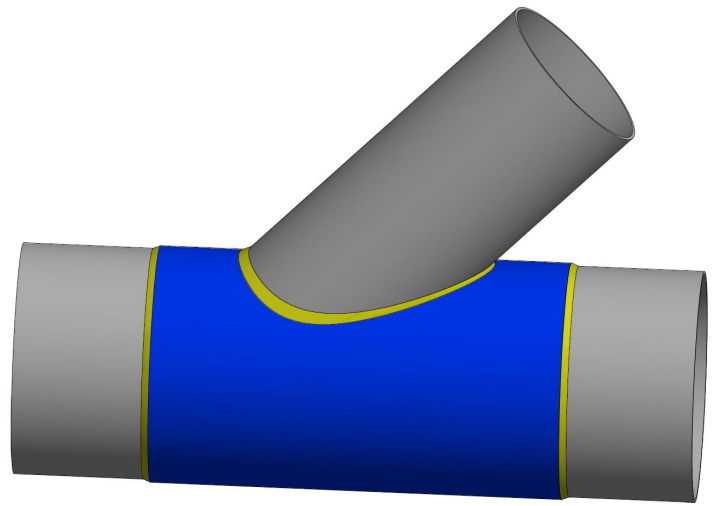

(b) Welding on reinforced tee.

Figure 3.16: Welding on reinforced tees

It must be noted that in practice most wrapper and collar reinforcing plates are made using the same diameter pipe as used for the barrel. The pipe for the wrapper plate is split in half in the longitudinal direction. The hole for the branch is then cut and the collar is cut as required. The two halves are then placed around the special, one on either side thereof and welded thereto. This can be seen in Figure 4.2b which shows the wrapper reinforced special tested in this study (Chapter 4). The wrapper is free from the barrel over the rest of its surface. Although certain areas of the barrel and reinforcing plate could be in contact the reinforcing plate is expected to only be in full contact 


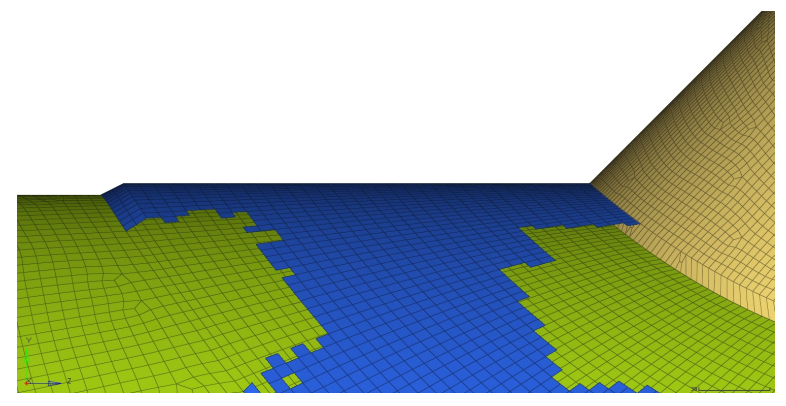

Figure 3.17: Intersection of reinforcing plate with barrel.

with the barrel upon radial expansion of the barrel under hydrostatic loading. In this study the contact interface was modelled as a fixed contact and zero relative displacement of the contact interface is enforced. The barrel is chosen as the master contact surface and the wrapper as the slave contact surface. The search distance (distance within which the normal projection of a slave node to a respective facet of the master surface must be to meet the contact condition criteria) was calculated as the sum of half the thickness of the barrel and the reinforcing plate.

Two discretization options for contact interfaces are available in Optistruct, namely Node-to-Surface (N2S) and Surface-to-Surface (S2S). With N2S discretization the contact surface is constructed by finding a master surface for each slave node that is within the specified search distance of the projection of the slave node onto the master surface. This option was used at the beginning of the study. It was found that this discretization method does not adequately represent the contact interface as seen in Figure 3.18a. With S2S discretization the contact surface is constructed by finding a master surface for each slave surface that is within the specified search distance of the projection of sample points (in the slave surface) onto the master surface. The projection of slave nodes onto the master surface $(\mathrm{N} 2 \mathrm{~S})$ is problematic if the meshes of the wrapper/collar and barrel do not match well which was the case in this study. Figure 3.18a shows the same wrapper reinforced tee modelled with S2S discretization where red is a high and blue a low stress area. It can be seen that the S2S discretization achieves a more consistent distribution of stresses on contact surfaces. As a result it was decided to instead use S2S discretization in this study.

One wrapper reinforced model was simulated with a sliding contact surface between the wrapper and barrel to allow relative movement between these two surfaces. The results showed that the model failed at the same internal pressure as when a fixed contact interface was used. The conclusion is made that the intersections between the barrel, branch and wrapper results in negligible relative deformation between the barrel and wrapper. 


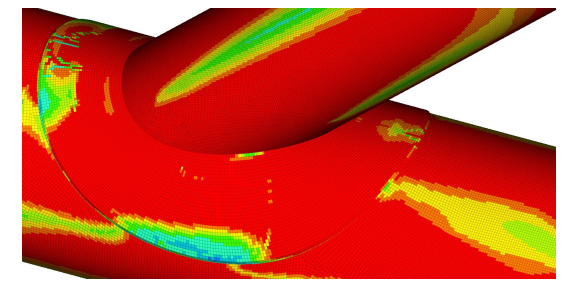

(a) Node-to-surface discretization.

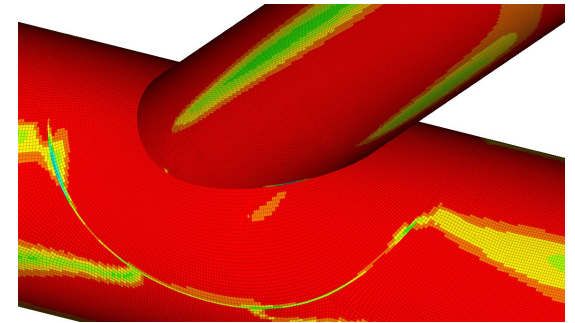

(b) Surface-to-surface discretization.

Figure 3.18: Contact surface discretization

\subsection{Numerical Results}

\subsubsection{M11 Designed Reinforcements}

The specials in this study are subjected to a triaxial state of stress and FE methods make it possible to approximate these stresses. The Von Mises stress theory is used to describe the triaxial stresses in the material.

It was concluded in this study that the intersection region is typically a high stress zone with Von Mises stresses well above the yield point of the steel. This is supported by the geometry of specials as described in Section 2.2 on page 6 and specifically in Figure 2.5 on page 9 . Under internal pressure and as a result of hoop stress the elliptical shape of the branch/barrel intersection is deformed into a circular ring. This results in high stresses in the intersection region and increasingly so towards the acute corner. From Figure 3.19a it can be seen that local yielding typically occurs long before failure of the special, in this case at an incremental load of 0.2 times the load at failure. Elements with a von Mises stress above the yield strength are shown in red and blue indicates a low stress. Yielding starts from the inside crotch corner of the intersection and then spreads outwards. The outward radial deformation of the intersection at point "A" is, however, significantly larger. The inside crotch corner area enters the plastic range long before the area around point "A".

In this study a variety of reinforced specials were modelled and numerically analysed. The thickness of the barrel and branch was chosen to be $1 / 100$ of the respective pipe diameter. The specials were designed in accordance with AWWA M11 and Tables 3.1 to 3.3 show the model parameters and a comparison between safety factors in accordance with M11 and the numerical results. From Table 3.1 it can be seen that the simulated safety factor for collar reinforced specials are lower than the M11 model. For the design of the wrapper and collar plate reinforced specials a yield strength of $290 \mathrm{MPa}$ was used in the AWWA M11 model. Therefore, it appears that the specials will fail at a lower internal pressure than what the M11 model suggests. The simulated safety factors are based on the assumptions and definition of failure 


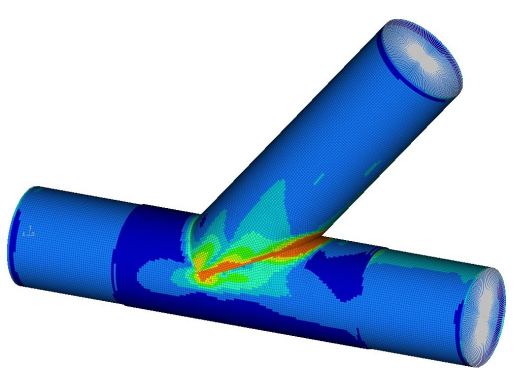

(a) Local yielding in the acute corner with a load factor of 0.2 times the load at failure.

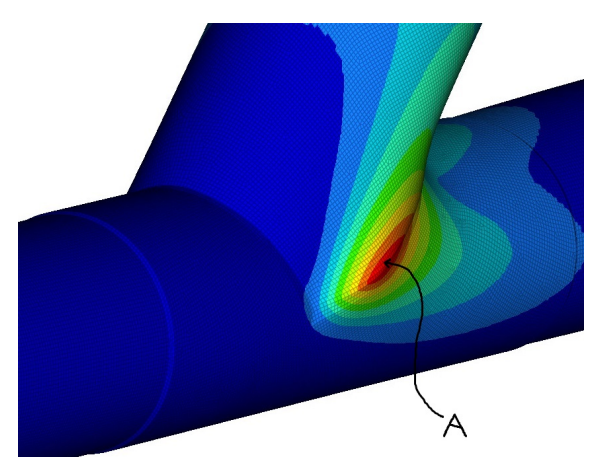

(b) Displacement at failure load.

Figure 3.19: Yielding and deformation of test piece

load as used in this study. The same conclusion is made for wrapper reinforced tees, as can be seen in Table 3.2. However, it is important to note that the simulated models are based on steel with elastic perfectly plastic behaviour and thus strain hardening is not accounted for. Figure 3.3 shows that, based on safety factors and the assumption used in this study, the M11 crotch plate design model seems to be conservative. The numerically calculated safety factors are in the order of five to six. The crotch plate reinforced specials were modelled with a yield strength of $207 \mathrm{MPa}$ as per AWWA M11. Table 3.3 shows the failure load and safety factor for each of the models at a yield strength of $207 \mathrm{MPa}$. The same specials were modelled with a yield strength of $290 \mathrm{MPa}$ to provide some indication of the effect of the yield strenght on the failure load as defined in this study. The simulated safety factors for the lower yield strength case seem to reduce with a factor equal to the reduction in yield strength. The simulation of the models in Table 3.3 with the actual yield strength of modern API 5L X42 steel used in this study is important in order to show simulated actual safety factors for such models designed by AWWA M11 and which is based on the lower yield strength of older steels. The simulated safety factors in Tables 3.1 to 3.3 represent only ductile failure simulated using EPP material behaviour. No allowance is made for other types of failure. 
CHAPTER 3. FINITE ELEMENT ANALYSIS OF CYLINDER-CYLINDER INTERSECTIONS

Table 3.1: Numerical results of collar plate reinforced specials.

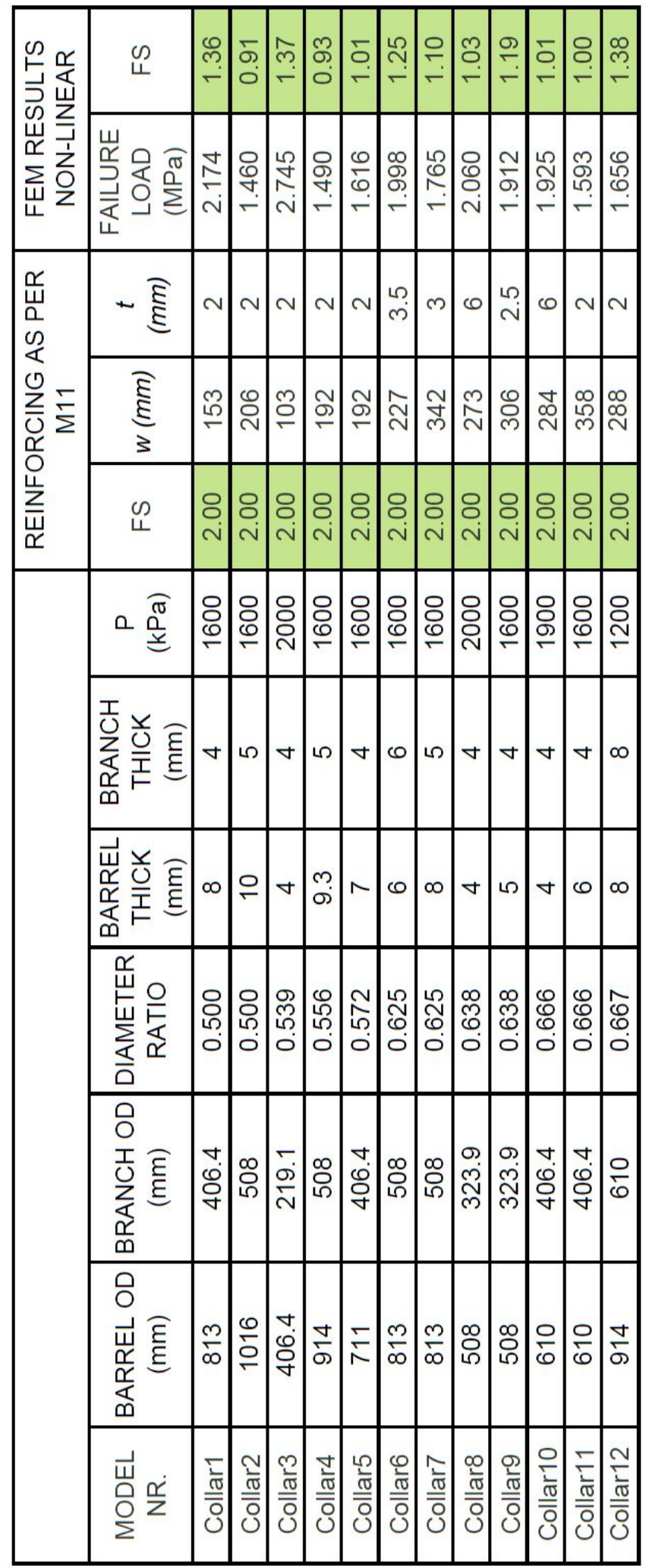


CHAPTER 3. FINITE ELEMENT ANALYSIS OF CYLINDER-CYLINDER INTERSECTIONS

Table 3.2: Numerical results of wrapper plate reinforced specials.

\begin{tabular}{|c|c|c|c|c|c|c|c|c|}
\hline \multirow{2}{*}{ 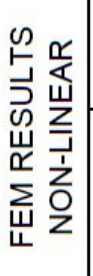 } & 足 & 志 & 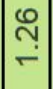 & $\stackrel{\text { N }}{\sim}$ & $\stackrel{g}{\stackrel{9}{-}}$ & $\stackrel{-}{\circ}$ & ஓ & $\stackrel{\text { ஜ̊ }}{\circ}$ \\
\hline & 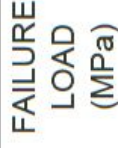 & \begin{tabular}{l}
$\mathscr{8}$ \\
$\stackrel{0}{0}$ \\
\hdashline
\end{tabular} & $\mid \begin{array}{c}\infty \\
\stackrel{\sim}{\sim} \\
\stackrel{\sim}{-}\end{array}$ & |ֶু & N & $\stackrel{\sim}{\stackrel{\sigma}{\sigma}}$ & & 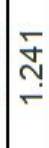 \\
\hline \multirow{3}{*}{ 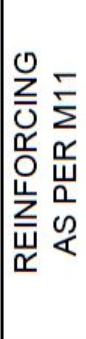 } & $\sim$ है & $\stackrel{n}{\sim}$ & $N$ & $N$ & $N$ & $N$ & & N \\
\hline & 3 है & 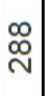 & $\mid \begin{array}{l}\infty \\
\infty \\
\sim\end{array}$ & 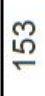 & & $\begin{array}{l}\infty \\
\stackrel{\infty}{*}\end{array}$ & ণ & 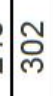 \\
\hline & 杄 & $\begin{array}{l}\text { \& } \\
\text { ì }\end{array}$ & $\begin{array}{l}\circ \\
\text { ì }\end{array}$ & 오 & d & i্ & ণ & 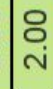 \\
\hline \multirow{2}{*}{\multicolumn{2}{|c|}{ 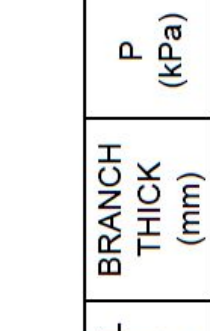 }} & \begin{tabular}{l}
$\stackrel{0}{0}$ \\
\hdashline \\
-
\end{tabular} & 움 & $\stackrel{\text { 음 }}{-}$ & $\stackrel{\text { o }}{\circ}$ & $\stackrel{\text { 우 }}{-}$ & 음 & $\stackrel{\text { ㅁ }}{\text { ㄱ }}$ \\
\hline & & $\begin{array}{l}n \\
+ \\
+\end{array}$ & 0 & t & 0 & \% & 0 & | \\
\hline & 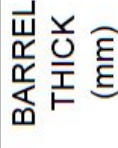 & 0 & 0 & | & 0 & م & 0 & | \\
\hline \multicolumn{2}{|r|}{ 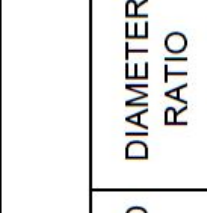 } & $\begin{array}{l}\stackrel{m}{0} \\
\stackrel{0}{o}\end{array}$ & 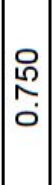 & $\begin{array}{l}\hat{2} \\
\stackrel{2}{0} \\
0\end{array}$ & 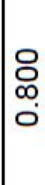 & & & 음 \\
\hline & 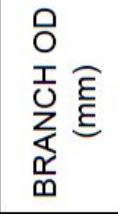 & 守 & $\frac{0}{6}$ & लू & $\begin{array}{l}\dot{y} \\
\dot{\theta} \\
\dot{\gamma}\end{array}$ & $\begin{array}{l}\dot{y} \\
\dot{\theta} \\
\dot{\gamma}\end{array}$ & & రి \\
\hline & 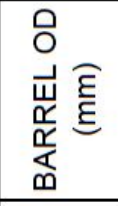 & 은 & $\frac{m}{\infty}$ & \begin{tabular}{|l}
+ \\
$\ddot{\theta}$ \\
$\dot{\sigma}$
\end{tabular} & 文 & & & 足 \\
\hline & $\begin{array}{l}\frac{\dot{\alpha}}{z} \\
\dot{山} \\
\dot{\partial} \\
\frac{0}{\Sigma}\end{array}$ & $\begin{array}{l}\frac{1}{0} \\
\frac{0}{0} \\
\frac{0}{0} \\
\frac{3}{3}\end{array}$ & $\mid \begin{array}{l}\frac{1}{0} \\
\frac{0}{2} \\
\frac{0}{0} \\
\frac{0}{3} \\
3\end{array}$ & $\begin{array}{l}\frac{2}{0} \\
\frac{2}{2} \\
\frac{2}{0} \\
\frac{\pi}{2} \\
3\end{array}$ & 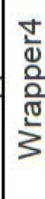 & 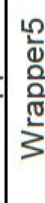 & \begin{tabular}{|l}
0 \\
$\frac{1}{2}$ \\
$\frac{0}{2}$ \\
$\frac{0}{30}$ \\
$\frac{10}{3}$
\end{tabular} & $\frac{1}{3}$ \\
\hline
\end{tabular}


CHAPTER 3. FINITE ELEMENT ANALYSIS OF CYLINDER-CYLINDER INTERSECTIONS

Table 3.3: Numerical results of crotch plate reinforced specials.

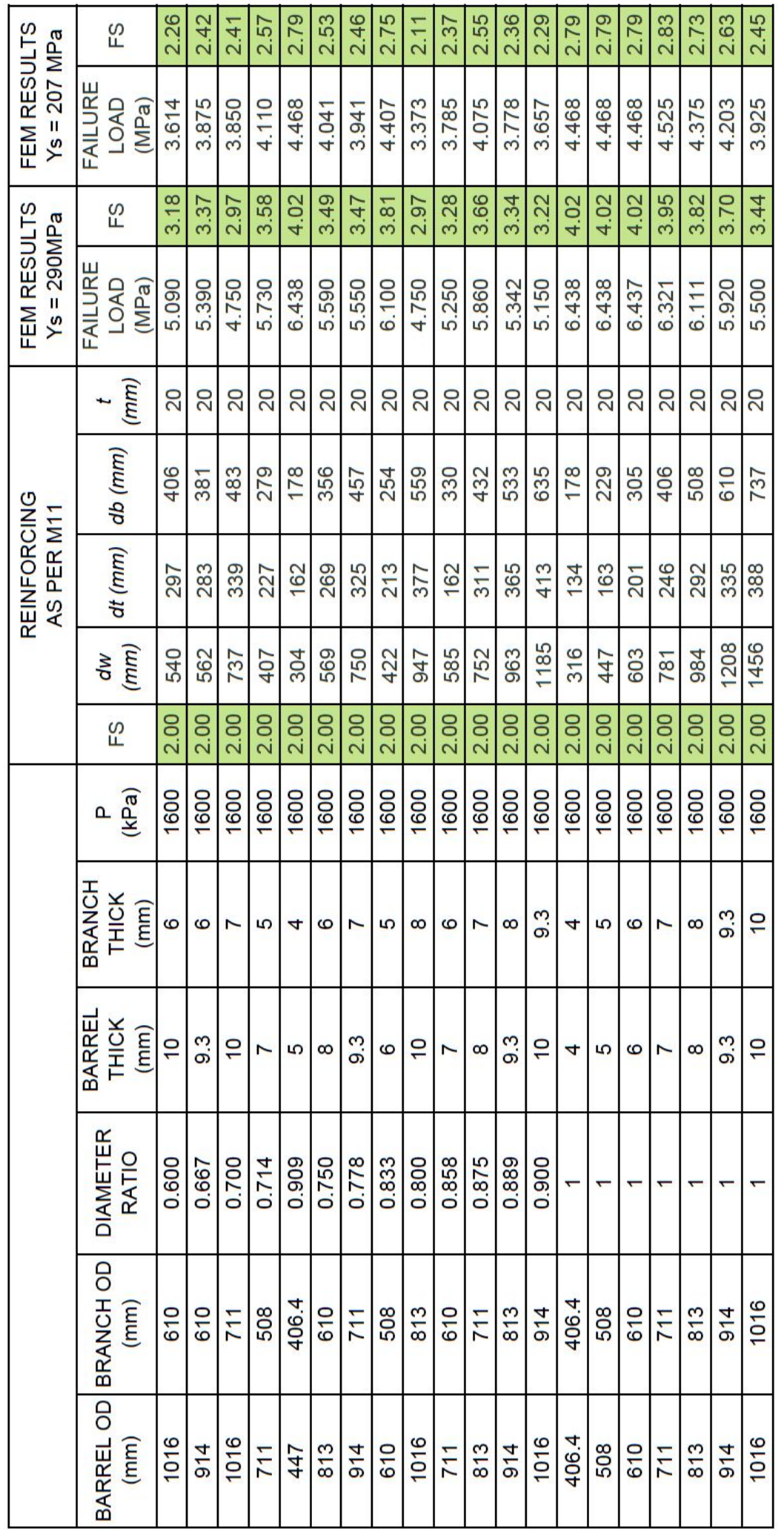


CHAPTER 3. FINITE ELEMENT ANALYSIS OF CYLINDER-CYLINDER INTERSECTIONS

\subsubsection{Adjustment factors for wrappers and collars}

Adjustment factors were considered in this study as a means of scaling/adjusting the design formulas provided by AWWA M11 in order to provide higher safety factors. In this study the focus was placed on adjustment factors for crotch plate designs. An introduction is given on the use of thicker collar plates as alternatives to thinner wrapper plates designed according to AWWA M11.

In this study the use of collar plates for specials with PDV values below 1050 and diameter ratios higher than 0.7 were investigated. The special defined in Table 3.4 was designed according to M11 and the simulated results shown in Figure 3.20a. The same special was then modelled with a collar plate of the same width as the wrapper plate $(w)$ and plate thickness of $3 \mathrm{~mm}$. Figure $3.20 \mathrm{~b}$ shows simulated results for the collar reinforced special. It can be seen that the failure load, shown in brackets, is slightly higher for the thicker collar reinforced special. This indicates that an adjusted plate thickness may result in collar plates being a sufficient reinforcement in certain applications. It is concluded that, in some cases, the additional stiffness added by the thicker collar plate may provide a stronger reinforcement than a wider wrapper plate. The development of factors to adjust the area replacement model suggested by AWWA M11 is however not an objective of this study as this design model was shown not to be overly conservative and therefore adjustments thereto is assumed to not provide much benefit in terms of reduced material quantity.

Table 3.4: Parameters for models in Figure 3.20.

\begin{tabular}{|c|c|c|}
\hline \multirow{2}{*}{\multicolumn{3}{|c|}{$\begin{array}{l}\text { PARAME IER } \\
\text { INPUTS }\end{array}$}} \\
\hline & & \\
\hline$D$ & 508 & $\mathrm{~mm}$ \\
\hline$d$ & 406.4 & $\mathrm{~mm}$ \\
\hline$T_{y}$ & 5 & $\mathrm{~mm}$ \\
\hline$t_{y}$ & 4 & $\mathrm{~mm}$ \\
\hline Angle & 45 & Degrees \\
\hline$P$ & 1.6 & $\mathrm{MPa}$ \\
\hline $\begin{array}{l}T_{\text {collar }} \\
\text { OUTPUTS }\end{array}$ & 3 & $\mathrm{~mm}$ \\
\hline PDV & 1040 & \\
\hline$w$ & 268 & $\mathrm{~mm}$ \\
\hline$T_{\text {wrapper }}$ & 2 & $\mathrm{~mm}$ \\
\hline
\end{tabular}




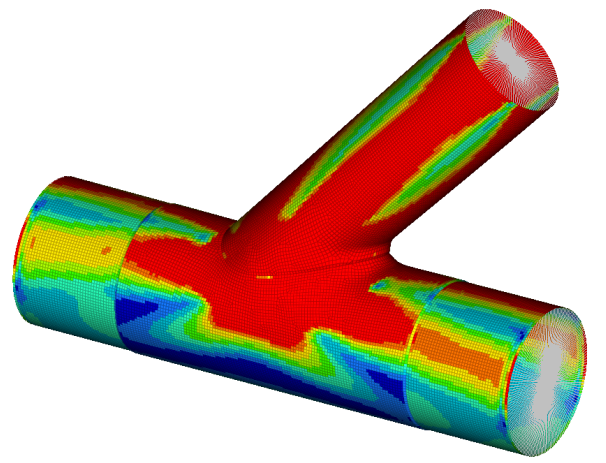

(a) Wrapper reinforced special (1.62 MPa).

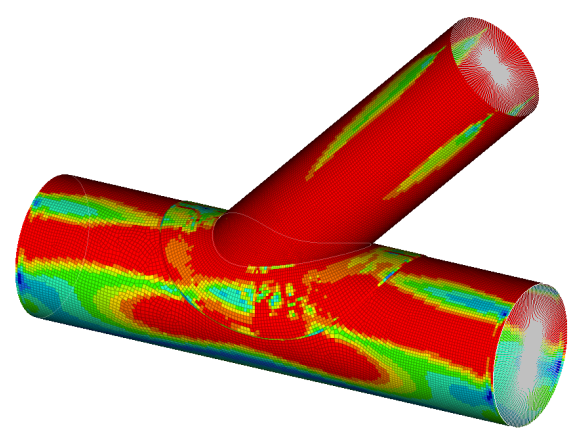

(b) Wrapper replaced with collar (1.78 MPa).

Figure 3.20: Comparison between wrapper and collar reinforced special with high diameter ratio.

\subsubsection{Adjusted Design Pressure Factor for Crotch Plates}

In an attempt to account for the higher yield strength of modern steels in M11 crotch plate designs, an adjusted design pressure shown in Equation 3.7.1, was evaluated in this study. The objective was to obtain an adjusted design pressure that could be used in the M11 design model, instead of the actual design pressure. Figure 3.21 shows the difference between two dimensionally identical crotch plate reinforced specials with different yield strengths. The model parameters are shown in Table 3.5. The yield strength of the model in Figure 3.21a is in accordance with M11 (207 MPa) and Figure 3.21b shows the same model with a yield strength of $290 \mathrm{MPa}$. The effect of yield strength can be seen in the failure load, shown in brackets for each load. As expected, the increased yield strength results in an increased safety factor.

$$
P_{e f f}=P \frac{Y_{M 11}}{Y_{s}}
$$

where

$Y_{M 11}=$ Yield strength as per Swanson et al. (1955)

$Y_{s}=$ Actual yield strength of the steel 
Table 3.5: Parameters for model in Figure 3.22.

\begin{tabular}{lcccc} 
PARAMETERS & $P_{\text {actual }}\left(Y_{M 11}\right)$ & $P_{\text {actual }}\left(Y_{s}\right)$ & $P_{\text {adjusted }}$ & UNIT \\
\hline$\underline{\text { INPUTS }}$ & 610 & 610 & 610 & $\mathrm{~mm}$ \\
$d$ & 610 & 610 & 610 & $\mathrm{~mm}$ \\
$T_{y}$ & 6 & 6 & 6 & $\mathrm{~mm}$ \\
$t_{y}$ & 6 & 6 & 6 & $\mathrm{~mm}$ \\
Angle & 45 & 45 & 45 & $\mathrm{Degrees}$ \\
PDV & 1952 & 1952 & 1393 & \\
Yield strength & 207 & 290 & 290 & $\mathrm{MPa}$ \\
$P_{a}$ ctual & 1.6 & 1.6 & 1.6 & $\mathrm{MPa}$ \\
$P_{\text {adjusted }}$ & & & 1.14 & $\mathrm{MPa}$ \\
$d_{w}$ & 603 & 603 & 490 & $\mathrm{~mm}$ \\
$d_{t}$ & 305 & 305 & 254 & $\mathrm{~mm}$ \\
$d_{b}$ & 201 & 201 & 178 & $\mathrm{~mm}$ \\
$t$ & 20 & 20 & 20 & $\mathrm{~mm}$
\end{tabular}

\section{OUTPUTS}

Simulated safety factor 2.79 4.01 3.56

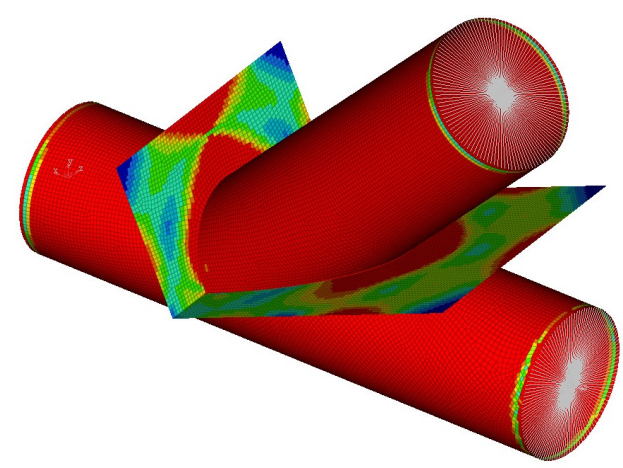

(a) Yield strength as per M11 (4.47 MPa).

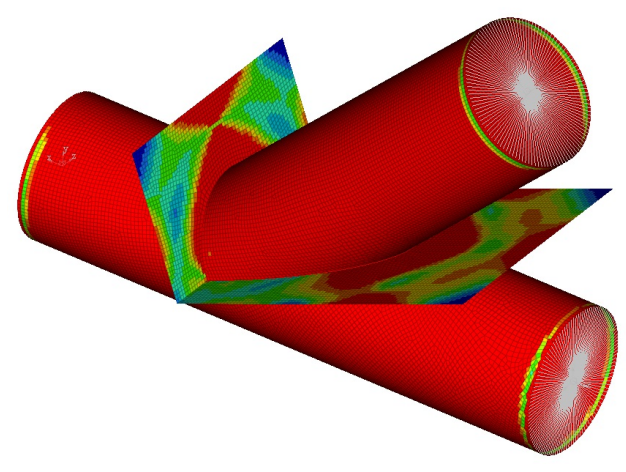

(b) Actual yield strength of steel (6.43 MPa).

Figure 3.21: Effect of yield strength on crotch plate reinforced special, where red is a high and blue a low stress. Although the failure load stress distribution is similar for both, the failure load is higher for an increase in yield strength.

The design pressure of the model shown in Figure 3.21b was adjusted using Equation 3.7.1 and the reinforcing redesigned, using M11, with the adjusted pressure. The adjusted crotch plate design was simulated and compared with the unadjusted design, as shown in Figure 3.21a. Column B and C in Table 3.5 shows the parameters for these two specials and Figure 3.22 shows the simulated results for the crotch plate reinforced special based on an adjusted pressure. The additional stiffness due to the larger crotch plate of the unadjusted design result in a slightly higher safety factor for this model. However, 
the safety factor of the adjusted pressure designed crotch plate is considered to be sufficient for steel pipe specials. A safety factor of 2 is generally used in practice for the design of steel pipe specials.

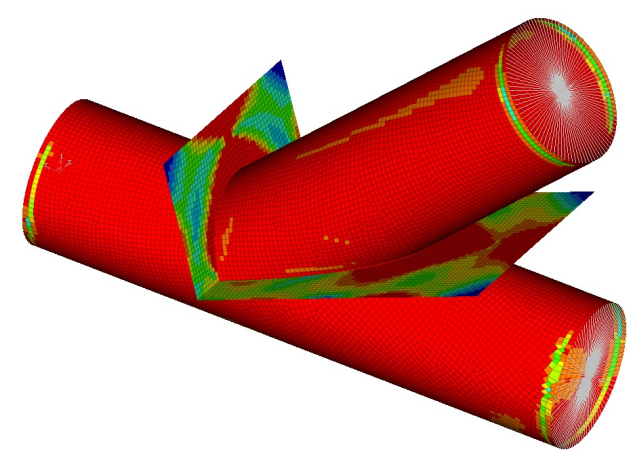

Figure 3.22: Adjusted pressure crotch plate design (5.72 MPa), where red is a high and blue a low stress.

\subsubsection{Adjusted Thickness Factor for Crotch Plates}

An alternative to the adjusted design pressure was considered by linearly adjusting the M11 obtained plate thickness with the change in yield strength instead of the pressure. Although this would not affect the PDV it would have an impact on the plate thickness when the PDV value indicates the use of a crotch plate. The objective is therefore to determine whether a higher yield strength permits a reduction in the plate thickness recommended by M11. The adjusted plate thickness was calculated using Equation 3.7.2. It is important to note that the yield strength is not affected by the thickness of the plate, rather the thickness provides stiffness to the special. When the design allows for increased deflection, a higher yield strength does, however, permit a reduction in stiffness. Subsequently, the adjusted thickness approach relies on an increase in yield strength to counter for a reduction in stiffness, due to material loss, and the assumption that increased deflection is acceptable in the design of steel specials. This is often the case with bulk water steel pipe specials.

The crotch plate reinforced special shown in Figure $3.21 \mathrm{~b}$ was modelled with an adjusted crotch plate thickness using Equation 3.7.2 and an actual yield strength of $290 \mathrm{MPa}$. Figure 3.23a shows the simulated model and failure load in brackets. It can be seen that the adjusted crotch plate design provides less stiffness to the special which results in a lower failure load. However, the safety factor of approximately 3.4 is considered to be sufficient for large diameter steel pipe special. The same special was also modelled with fully constrained end points (compared to only one end of the barrel constrained) to provide some indication on the effect of different boundary conditions on reduced crotch plate thicknesses. The simulated model is shown in Figure 3.23b 
with the failure load in brackets. It can be seen that with all ends of the special fully constrained the failure load is slightly less. This highlights the importance of accurately defining the boundary conditions.

$$
t_{a d j}=t \frac{Y_{M 11}}{Y_{s}}
$$

where

$$
\begin{aligned}
Y_{M 11} & =\text { Yield strength as per Swanson et al. (1955) } \\
Y_{s} & =\text { Actual yield strength of the steel }
\end{aligned}
$$

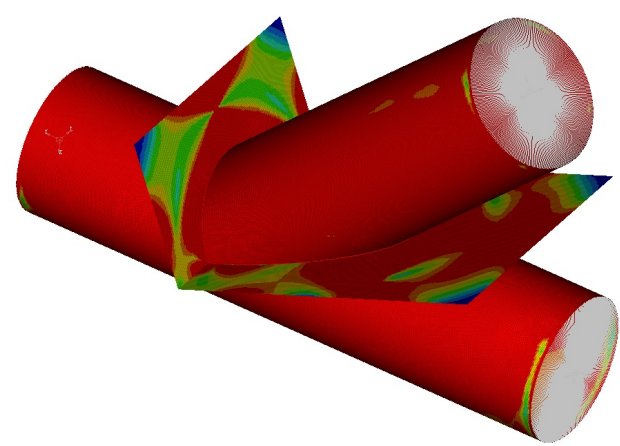

(a) One end of barrel fully constrained (5.46 MPa).

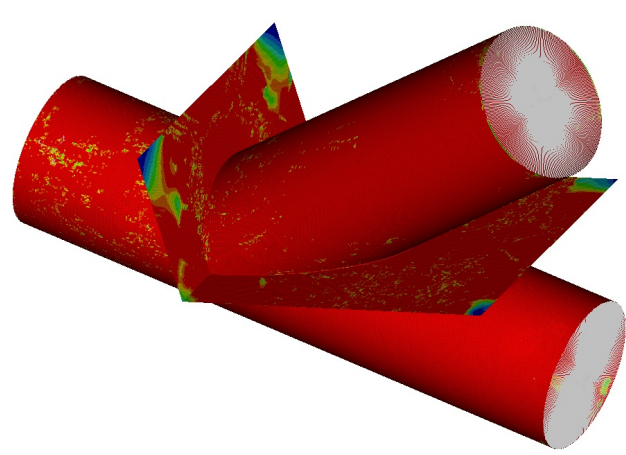

(b) All ends fully constrained (4.75 MPa).

Figure 3.23: Boundary condition effects on adjusted thickness crotch plates, where red is a high and blue a low stress.

\subsection{Model Variations}

Multiple model variations are possible with reinforced specials to better suit the application and pipework configuration or as a result of different manufacturing procedures. Different intersection angles, curving of the crotch plate, integral versus pad thickening (refer to Fig. 2.11) and fixing the ends of the special are amongst some of the possible variations. These and other variations are discussed in this study to provide some indication of the impact thereof and serving as a guideline to designers.

Crotch plate reinforced tees do not have contact surfaces, only intersecting edges. The curved surface of a crotch plate, for an unequal tee, has a complex geometry due to the ellipsoidal shaped curvature of the branch/barrel intersection and hence it is difficult to model. Manufacturing of these plates are not exact as the curvature and shape typically varies from manufacturer to manufacturer, largely due to the complexity thereof. Figure 3.24 shows the 
angle of the reinforcing plate on both the acute and obtuse angle side of the branching. The angle of the reinforcing plate is not explicitly given by the AWWA M11 code and it is challenging to curve the reinforcing plate exactly even if such angles were defined. The AWWA M11 code does provide a radius for the plate. In this study the curvature of the reinforcing plates were chosen such that it is tangent to the curvature at the point where it moves away from the barrel-branch intersection. Section 3.8 deals with the impact of different crotch plate curvatures on pipe stresses. The amount of welding required to fix the three components to each other at a single intersecting point will also vary from welder to welder, mainly due to the welding of the branch/barrel intersection and the matching curvature of the crotch plate thereto. The larger the gap between the crotch plate and the branch/barrel intersection the more welding is required. The amount of welding required could increase the possibility of welding defects. The quality of the weld has a direct impact on the safety factor of the tee and it is left to the designer to decide on a sufficient factor of safety for each application to allow for welding defects. Weld inspections and testing are recommended to avoid significant defects and avoid the necessity for a factor of safety higher than two. In this study the effects of welds are ignored with the assumption that no welding defects exist.

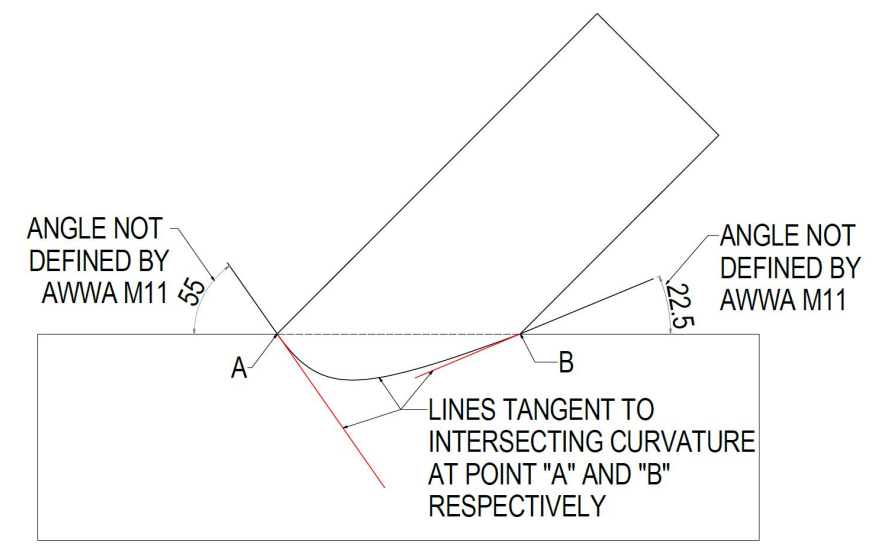

Figure 3.24: Curvature of crotch plates for unequal tees.

The intersection angle typically varies between 30 degrees and 90 degrees with 30, 45 and 90 degree intersections being the most widely used. For angles smaller than 30 degrees it becomes challenging to achieve an acceptable weld in the acute corner. Figure 3.25 shows the impact of the intersection angle on a typical wrapper reinforced special, with the internal pressure, just prior to failure of each special, shown in brackets. It can be seen that the higher stresses are typically on the inside crotch corner (refer to Figure 2.6) side of the intersection. The same applies for collar and crotch plate reinforced specials.

With equal diameter specials reinforcing wrapper plates can be shaped with either a smooth curvature or a V-shaped curvature. Figure 3.26 shows the two 


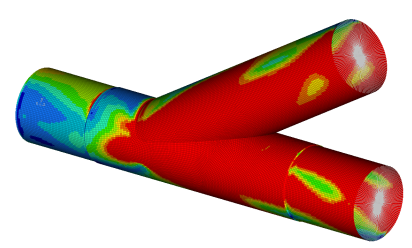

(a) 30 Degree wrapper reinforced tee (1.54 MPa).

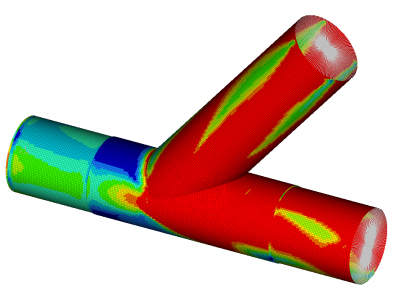

(b) 45 Degree wrapper reinforced tee (2.03 MPa).

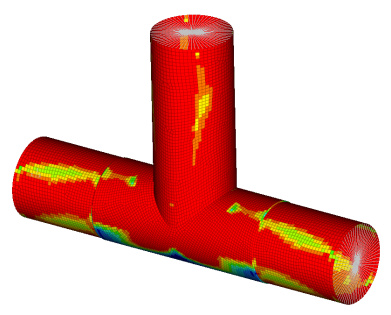

(c) 90 Degree wrapper reinforced tee (4.17 MPa).

Figure 3.25: Impact of intersection angle on stresses, where red is a high and blue a low stress.

shapes and corresponding stress contours for the same boundary conditions and loads with red a high and blue a low stress. The typical internal pressure at failure of each special is shown in brackets. The additional material does not seem to have a significant impact on the failure load in this case. Figure $3.26 \mathrm{~b}$ is the test special described further in Section 4 and Figure 3.19b shows the area where the largest deformations occur on the test special.
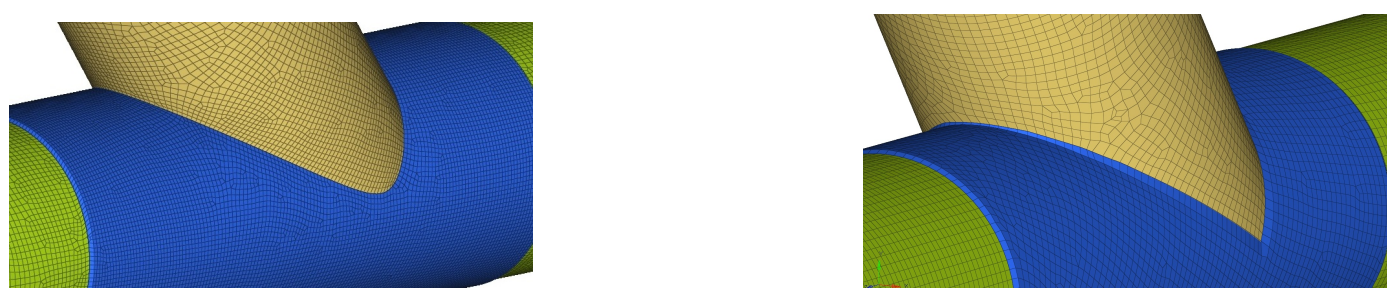

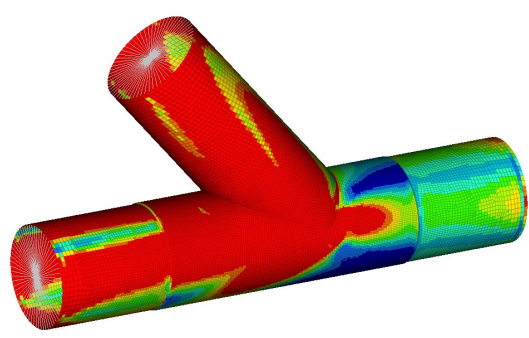

(a) Smooth (3.33 MPa)

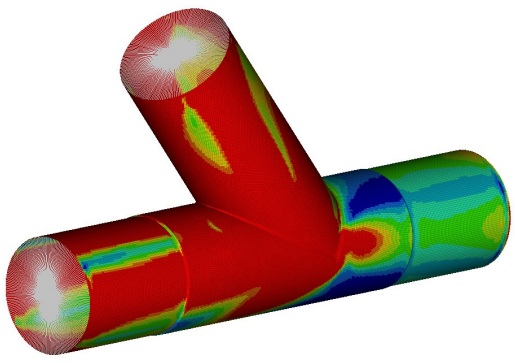

(b) V-shape (3.63 MPa)

Figure 3.26: Different wrapper shapes on equal diameter specials and corresponding stress contours.

Integral reinforcing can be used as an alternative to pad reinforcements such as wrappers and collars. The required reinforcing area is provided by increasing the barrel or branch thickness. The increased thickness need not be for the entire length of the barrel/branch as shown in Figure 2.10 on page 
13. A thinner pipe can be welded on either side of the thickened pipe section. Figure 3.27 presents a wrapper reinforced model with integral and pad reinforcing respectively. The model parameters are shown in Table 3.6. In this study the integral reinforcing was defined over the full lenghth of the barrel. The simulated failure loads, shown in brackets in Figure 3.27, indicate that the pad reinforced special fail at a slightly higher internal pressure. This is partly due to the fact that the pad reinforcement slightly laps onto the branch.

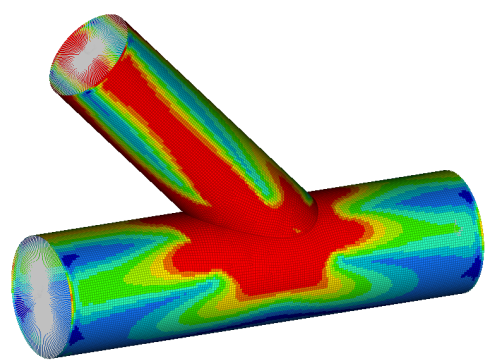

(a) Integral (1.52 MPa)

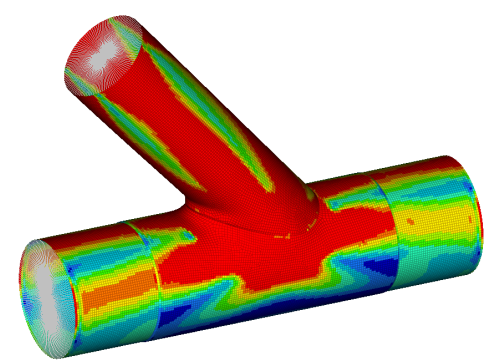

(b) Pad (1.62 MPa)

Figure 3.27: Integral vs. pad reinforcing.

Table 3.6: Parameters for models in Figure 3.27.

\begin{tabular}{lccl} 
PARAMETER & INTEGRAL & PAD & UNIT \\
\hline$D$ & 508 & 508 & $\mathrm{~mm}$ \\
$d$ & 406.4 & 406.4 & $\mathrm{~mm}$ \\
$T y$ & 7 & 5 & $\mathrm{~mm}$ \\
$t y$ & 4 & 4 & $\mathrm{~mm}$ \\
Angle & 45 & 45 & Degrees \\
PDV & 1040 & 1040 & \\
Yield strength & 290 & 290 & $\mathrm{MPa}$ \\
Safety factor & 3 & 3 & \\
Allowable yield strength & 97 & 97 & $\mathrm{MPa}$ \\
$P$ & 1.6 & 1.6 & $\mathrm{MPa}$ \\
Barrel length & 1900 & 1900 & $\mathrm{~mm}$ \\
$w$ & 0 & 268 & $\mathrm{~mm}$ \\
Wrapper total length & 0 & 1110 & $\mathrm{~mm}$ \\
$t$ & 0 & 6 & $\mathrm{~mm}$ \\
\hline
\end{tabular}

In Section 3.4 it was pointed out that the curvature of crotch plates (Fig. 3.28) is not explicitly defined by the AWWA M11 code. However, the curvature could have some, albeit little, impact on stresses, as can be seen in Figure 3.29. Only the curvature of the crotch plate was changed while all other parameters of the special is identical for the three cases. The internal pressure just before 
failure of the special is shown in brackets for each case. The numerical analysis shows that the crotch plate is most effective when curved tangential to the barrel/branch intersection (refer Fig. 3.24). In practice this is typically how crotch plates are manufactured as it is the natural curvature of the plate after being welded to the intersection.

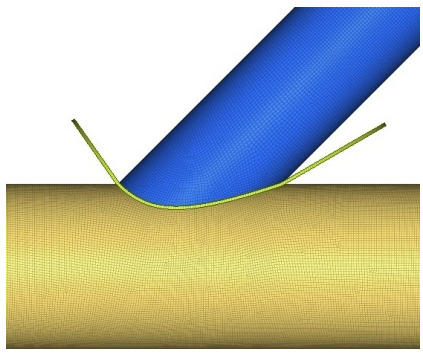

(a) Crotch plate curving upwards.

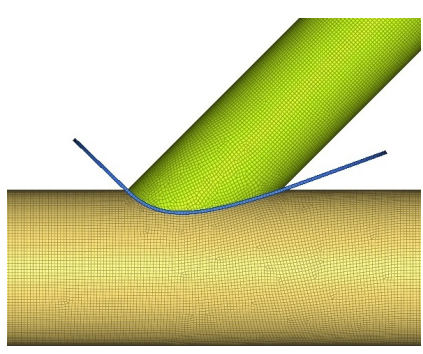

(b) Crotch plate tangential to bar$\mathrm{rel} /$ branch intersection.

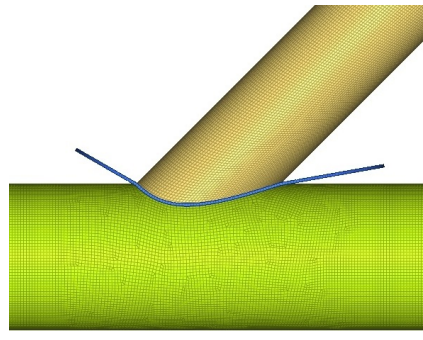

(c) Crotch plate curving downwards.

Figure 3.28: Difference in crotch plate base and wye exit angles.

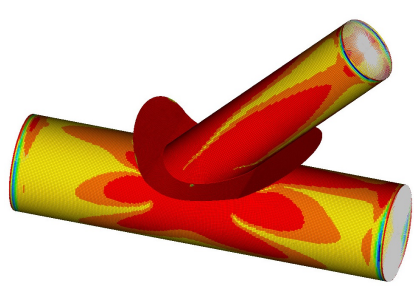

(a) Crotch plate curving upwards (5.2 MPa).

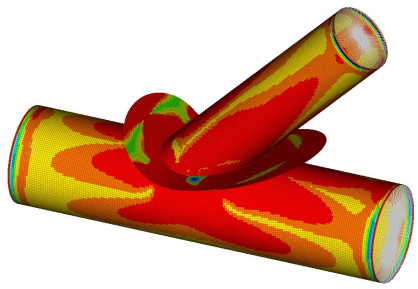

(b) Crotch plate tangential to bar$\mathrm{rel} /$ branch intersection (5.4 MPa).

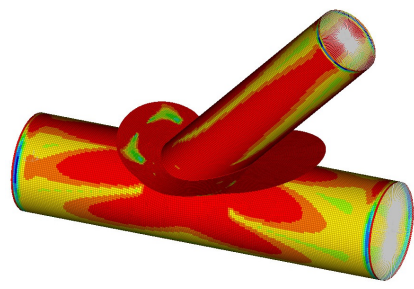

(c) Crotch plate curving downwards (5.0 MPa).

Figure 3.29: Impact of crotch plate curving on stresses, where red is a high and blue a low stress.

In Section 3.5.1 the impact of different boundary conditions on stresses were considered. It is important to note that boundary conditions will vary from one installation to another. In certain applications specials are installed between three points that are fixed in position by some form of pipe support. In this case the ends are restricted from moving in the axial direction of the respective pipe. Where specials are installed in rigid pipework assemblies, with no flexible connections, the same boundary conditions could apply. The crotch plate reinforced tee shown in Figure 2.13 on page 15 is fixed on one end of the barrel and secured on the other two ends by means of restraining bolts. Movement in the axial direction of each pipe is therefore very limited 
and even more so if the restraining flanges had securing nuts on either side of it as shown in Figure 3.30. In this case it would be more representative of the physical installation to model the special with all three open ends being constrained from any movement.

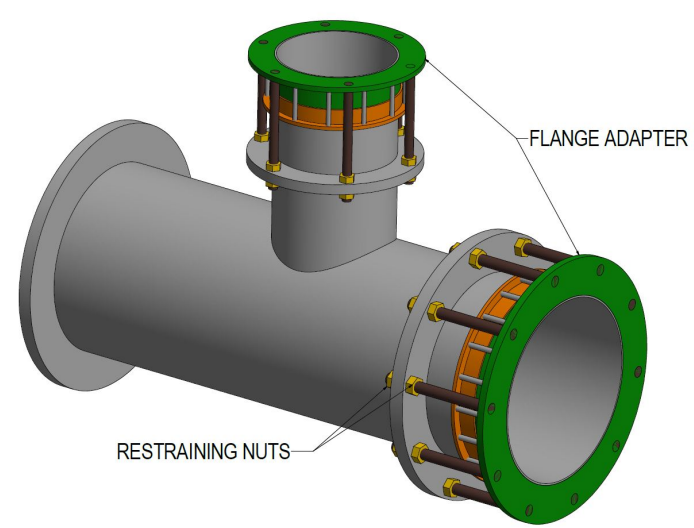

Figure 3.30: Crotch plate reinforced tee restrained on all three open ends.

\subsection{Alternative Reinforcements}

Reinforcements not designed in accordance with AWWA M11 are also encountered for specials in bulk water pipelines. In this study an introductory analysis on a number of different types of reinforcements is performed to provide some indication of the safety factors obtained with these methods as well as the suitability thereof.

\subsubsection{Internal reinforcements}

Internal reinforcements are sometimes used in practice, mostly in the form of a round pipe welded to the barrel and normal to the barrel's axial direction. With the special subjected to internal pressure the internal reinforcement is largely in tension making it more effective than external reinforcements providing stiffness through a clamping action. Internal reinforcements in the form of round pipes are flow restrictors and hence causes hydraulic losses. In order to minimize these losses the use of internal reinforcements in the form of flat plates were considered in this study. It is suggested in this study that flat plate internal reinforcements could be designed in conjunction with computation fluid dynamics to not only reinforce steel specials, but also improve flow lines and reduce head losses.

Internal bracing in the form of a flat plate was considered in this study. The wrapper plate on the test piece model was replaced with an internal flat plate through the centerline of the branch as shown in Figure 3.31. The model 
parameters are given in Table 3.7. With the tee subjected to internal pressure the reinforcing plate is largely in tension. It can be seen in Figure 3.31c that the plate is ineffective in dealing with the outward deformation of the barrel as it does not provide stiffness to the critical region of the intersection. Moving the plate to the centerline of the barrel seems to be even less effective as shown in Figure 3.32 as it does not provide stiffness to the acute corner (critical region) of the intersection. It is expected that head losses will increase significantly when moving the internal plates closer to the acute corner of the intersection due to a larger area of the barrel (and possibly branch) being obstructed. The selection and placement of internal reinforcing plates in this study do not seem to be effective and the optimal design and placement thereof are not an objective of this study. Further work is needed to determine the optimal shape and placement of internal plates to act as both a reinforcement and flow straightener. Subsequently, the effectiveness of such internal reinforcing plates should be determined. It is suggested in this study that internal reinforcing plates could possibly be designed in conjunction with computation fluid dynamics to also improve flow lines and reduce head losses.

Table 3.7: Parameters for wrapper and branch internal plate reinforced tee.

\begin{tabular}{lccl} 
PARAMETER & WRAPPER & BRANCH INTERNAL PLATE & UNIT \\
\hline$D$ & 610 & 610 & $\mathrm{~mm}$ \\
$d$ & 610 & 610 & $\mathrm{~mm}$ \\
$T y$ & 6 & 6 & $\mathrm{~mm}$ \\
$t y$ & 6 & 6 & $\mathrm{~mm}$ \\
Angle & 50 & 50 & Degrees \\
PDV & 1039 & 290 & $\mathrm{MPa}$ \\
Yield strength & 290 & & \\
Safety factor (M11) & 4.65 & 1 & $\mathrm{MPa}$ \\
$P$ & 1 & & $\mathrm{~mm}$ \\
$w$ & 397 & 4 & $\mathrm{~mm}$ \\
$t$ & 6 & 2.26 & \\
Simulated safety factor & 3.2 & & \\
\hline
\end{tabular}




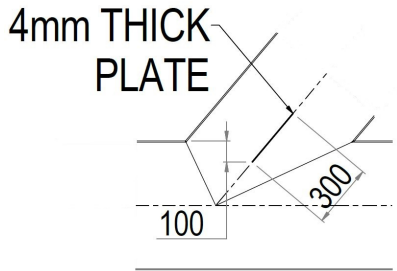

(a) Dimensions of internal plate.

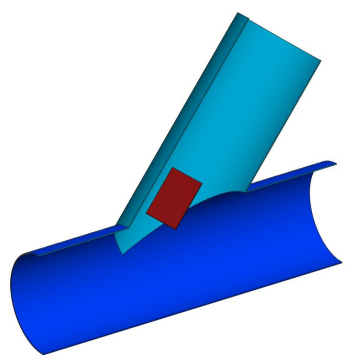

(b) $3 \mathrm{D}$ view.

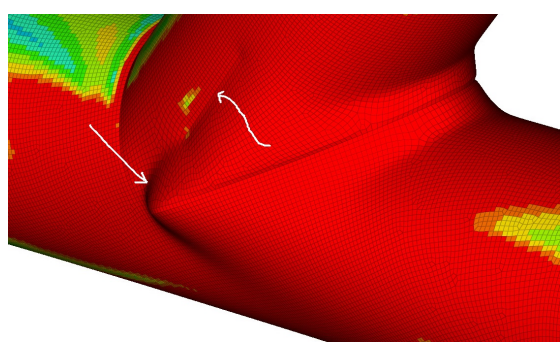

(c) Simulated deformed model at a failure load of $2.26 \mathrm{MPa}$.

Figure 3.31: DN600/DN600 test piece with wrapper plate replaced by branch internal reinforcing plate.

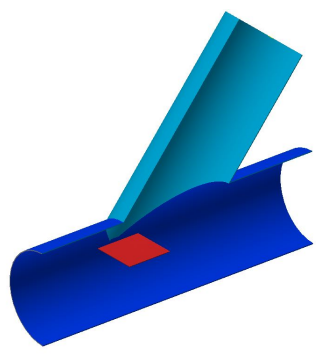

(a) 3D view.

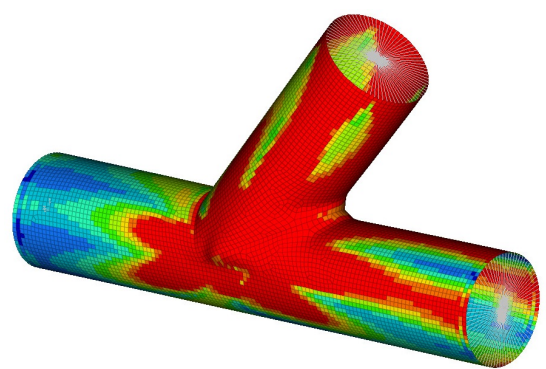

(b) Simulated deformed model at a failure load of $1.58 \mathrm{MPa}$.

Figure 3.32: Internal reinforcing plate moved to centerline of barrel.

\subsubsection{EN13480-3 crotch plate}

The EN13480-3 crotch plate design code was introduced in Section 2.3. As part of this study a FE analysis of a special (see Figure 3.33), designed and manufactured according to this method, was done to provide some indication on the safety factor of a typical special designed in accordance with this code.

The special was designed for an internal design pressure of $1.6 \mathrm{MPa}$ and a safety factor of 3 . It is manufactured from stainless steel pipe with a yield strength of $205 \mathrm{MPa}$. A nonlinear analysis, as described in Section 3.6, was performed and the load factor, just prior to non-convergence of the solution, recorded. The Young's modulus and Poisson's ratio was assumed to be $193 \mathrm{GPa}$ and 0.3 respectively. Non-convergent nonlinear iterations occured at an internal pressure loading of $3.57 \mathrm{MPa}$. The stress distribution at this load factor is shown in Figure 3.34 with red being a high and blue a low stress. The safety factor, for the given design pressure of $1.6 \mathrm{MPa}$, is calculated at 2.125 and therefore the simulated model shows that the EN13480-3 method is somewhat unconservative for this special. It is not an objective of this study 
to elaborate on the effectiveness of this design standard in detail. Rather, a typical design in accordance with this standard was modelled to provide some indication of the simulated safety factor compared to the designed safety factor.

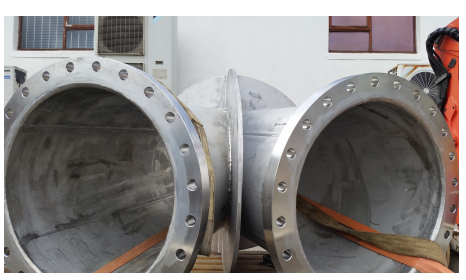

(a) Acute corner with reinforcing plate.

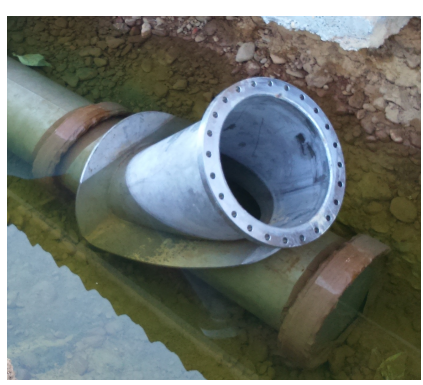

(b) Installed underground in pump station suction manifold.

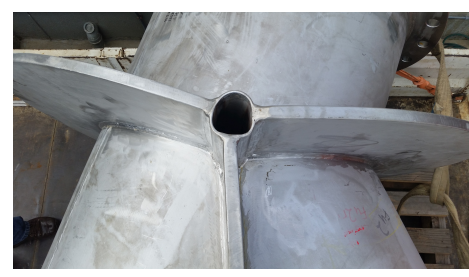

(c) Intersection of three plates with stabilizer bar.

Figure 3.33: DN800/DN800 crotch plate reinforced tee in accordance with EN13480-3.

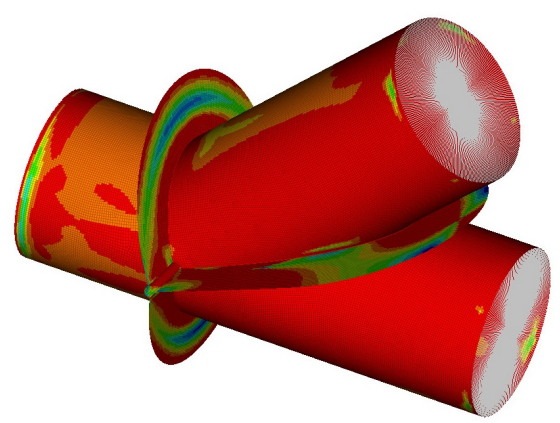

Figure 3.34: Simulated model of EN13480-3 crotch plate reinforced special.

\subsubsection{Gusset plates}

Another common reinforcing method is the use of gusset plates. Figure 3.35 shows a typical gusset plate reinforced special for pipes in accordance with the SANS 719 standard ((SANS 719 Standard)). However, supporting documentation on the design of such plates could not be found and therefore a conclusion could not be made on the safety factors provided by this standard. In this study a gusset reinforced tee was simulated to provide some comparison between gusset plates and M11 defined crotch plates. All ends of the tee were fully constrained and a pressure force applied to the internal surfaces of the branch and the barrel. Figure 3.36 shows simulated stresses for the gusset 
reinforced tee defined in Table 3.8. AWWA M11 shows that a crotch plate is required with the dimensions given in Table 3.8. The simulated crotch plate reinforced tee is shown in Figure 3.37. Less steel is required for the gusset plate and therefore it appears that the gusset plate design is more efficient. For different safety factors and tee dimensions crotch plates could prove to be more efficient. Further FE modelling is required to conclude on the use of gusset plates instead of crotch plates for larger tees and higher internal pressures. The objective of this study is not to elaborate in detail on the effectiveness of gusset plates for a wide range of applications, but rather to provide some insight on the simulated safety factor for a typical gusset plate design.

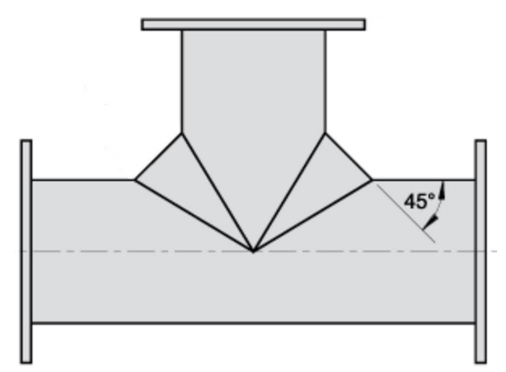

Figure 3.35: SANS 719 related gusset reinforced tee (Quality Tube Services)

Table 3.8: Parameters for models in Figure 3.20.

\begin{tabular}{lccl} 
PARAMETER & M11 CROTCH PLATE & GUSSET & UNIT \\
\hline$D$ & 914 & 914 & $\mathrm{~mm}$ \\
$d$ & 610 & 610 & $\mathrm{~mm}$ \\
$T y$ & 8 & 8 & $\mathrm{~mm}$ \\
$t y$ & 8 & 8 & $\mathrm{~mm}$ \\
Angle & 90 & 90 & Degrees \\
$P$ & 3.28 & 3.28 & $\mathrm{MPa}$ \\
PDV & 1335 & & \\
Gusset plate thickness & & 8 & $\mathrm{~mm}$ \\
$d w$ & 417 & & $\mathrm{~mm}$ \\
$d t$ & 311 & & $\mathrm{~mm}$ \\
$d b$ & 417 & & $\mathrm{~mm}$ \\
$t$ & 20 & & $\mathrm{~mm}$ \\
$S F$ & & & $\mathrm{~mm}$ \\
\hline
\end{tabular}




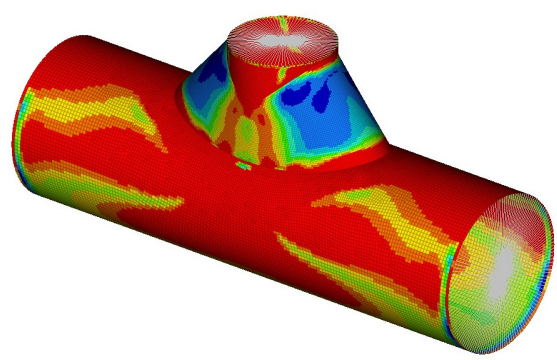

(a) Gusset shown.

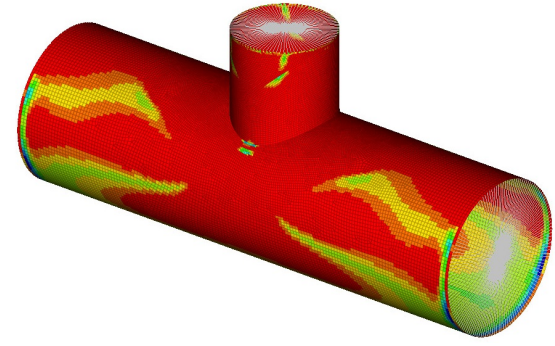

(b) Gusset not shown.

Figure 3.36: Gusset reinforced tee with a failure load of 4.66 MPa.

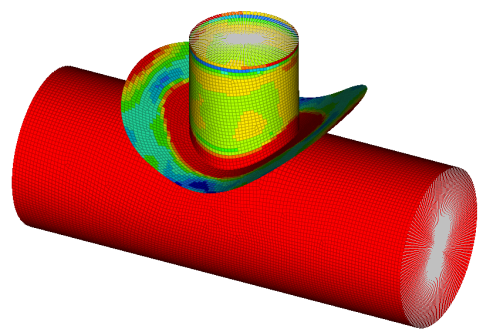

Figure 3.37: Simulated stress for crotch plate reinforced tee at a failure load of $5.87 \mathrm{MPa}$.

\subsection{Modelling Conclusion}

In this chapter a number of important factors for the simulation of cylindercylinder intersections are considered. Some background to numerical modelling and pre-processing is given and the classification of problems are discussed with emphasis on linear and nonlinear behaviour. Geometry simplifications are emphasized and the process of setting up a finite element analysis for steel specials are described. Element selection and mesh refinement was discussed with emphasis on the impact on convergence. Assumptions used in this study are listed and the process of midsurfacing and element overlap was described. The contact between surfaces was considered and the difference between node-tosurface and surface-to-surface discretization was described. Consideration was given to boundary conditions and typical effects on failure loads were shown. Crotch plate curvature was discussed and the effect on failure loads were considered. Alternative types of reinforcements were considered and simulated with $\mathrm{FE}$ analyses. 


\section{Chapter 4}

\section{Physical Testing}

\subsection{General and Objectives}

The physical test is to provide validation of the typical assumptions and procedures followed in the simulated FE models (refer to Chapter 3). Strains recorded during the hydrostatic test should be within $20 \%$ of the numerical model.

To validate a representative $\mathrm{FE}$ model an equal diameter wrapper reinforced lateral tee, defined in Table 4.1, was manufactured and hydrostatically tested. The objective was firstly to determine the failure load of the special and compare this to the simulated failure load of the same special. Furthermore, $0 / 90$ degree rosette strain gauges were used to measure strains in the pipe walls.

Table 4.1: Test piece parameters with M11 design.

\begin{tabular}{lcl} 
PARAMETER & VALUE & UNIT \\
\hline$D$ & 610 & $\mathrm{~mm}$ \\
$d$ & 610 & $\mathrm{~mm}$ \\
$T y$ & 6 & $\mathrm{~mm}$ \\
$t y$ & 6 & $\mathrm{~mm}$ \\
Angle & 50 & Degrees \\
$P$ & 1.6 & $\mathrm{MPa}$ \\
PDV & 1040 & \\
Yield strength & 290 & $\mathrm{MPa}$ \\
Safety factor & 4.65 & \\
Allowable yield strength & 62.37 & $\mathrm{MPa}$ \\
$w$ & 397 & $\mathrm{~mm}$ \\
$T$ & 6 & $\mathrm{~mm}$ \\
\hline
\end{tabular}




\subsection{Test Piece Manufacturing}

The test piece was manufactured from carbon steel pipe (SANS 719 Grade B) and carbon steel flanges (SANS 1123 Table 1600/8). The branch and barrel were welded together as shown in Figure 4.1. The wrapper was manufactured using the same pipe as used for the branch and barrel by splitting it in half. The two halves were then placed around the barrel, one on each side, and welded to the barrel and barrel/branch intersection as can be seen in Figure 4.2. The longitudinal butt welds make up for the material loss as a result of splitting the wrapper pipe in two halves.

Slip-on flanges were welded to the three open pipe ends. Blank flanges were bolted to the slip-on flanges with compressed fiber gaskets in-between to provide a watertight seal under internal pressure. All welds were visually inspected for pinholes and other visible welding defects after manufacturing of the special to avoid failure due defects as far as possible. The cost of more advanced tests such as radiographic testing prevented the use of these tests.

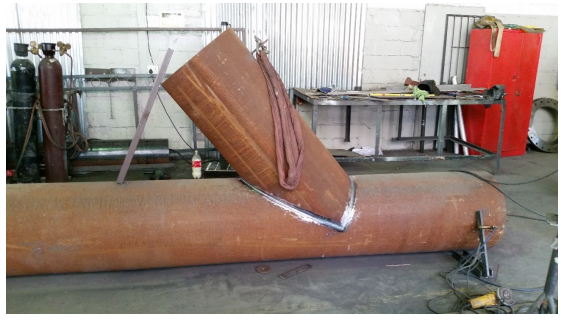

(a) Lateral tee test piece with branch welded to barrel.

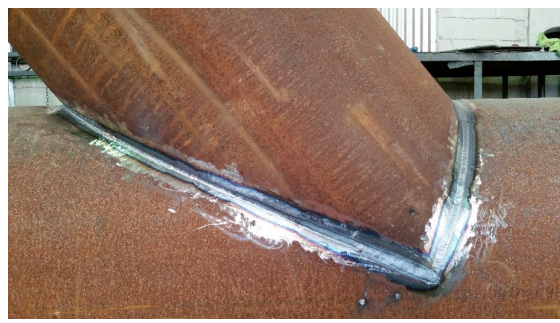

(b) Barrel/branch intersection.

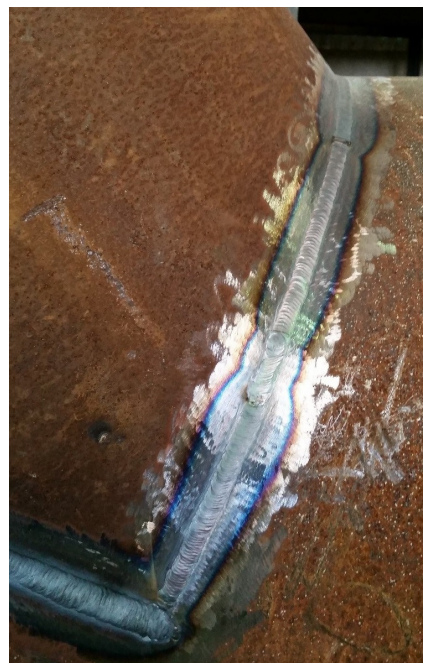

(c) Barrel/branch intersection weld with heat affected zone visible.

Figure 4.1: Welded test piece prior to addition of reinforcing plate. 


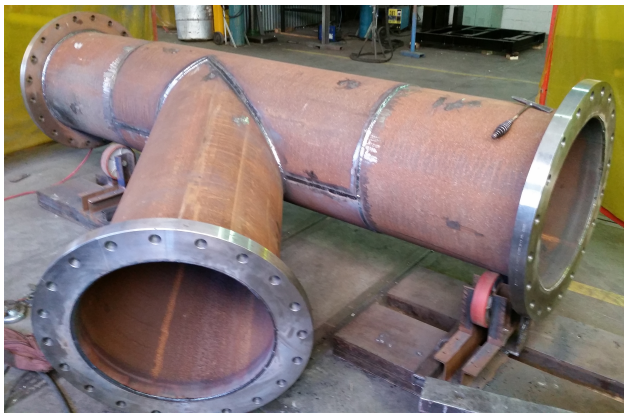

(a) Wrapper halves welded to the barrel.

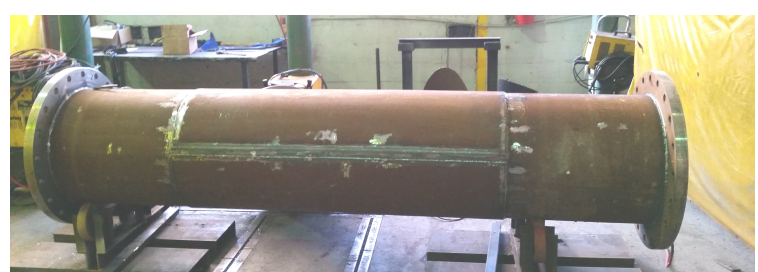

(b) Longitudinal weld on opposite side of branch.

Figure 4.2: Wrapper welded to test piece.

\subsection{Experimental Setup}

The test piece was set up in the upright position with all three ends simply supported as shown in Figure 4.3. The barrel was supported by the floor and the branch by a vertical support on either side. The three ends were therefore free to move relative to each other. As all internal forces balance each other out under hydrostatic loading, the test piece does not have to be restrained i.e. fixed to a rigid support. The vertical slide supports only prevent the test piece from falling over. Although the test could also have been performed with the test piece in the horisontal position it was easier to fill with water in the vertical position.

Strain gauges were applied to the internal and external surfaces of the test piece. The wires for the gauges applied to the internal surfaces were sealed using compression glands with tapered rubber washers as shown in Figure 4.4. The internal strain gauges were sealed off with rubber tape and a plastic cover. These strain gauges were placed directly opposite external strain gauges. It is expected that the strains measured by the external strain gauges would differ from the internal gauges due to the thickness of the branch and/or barrel. Even more so where the external gauges are applied to the wrapper. This is partly because of the increased distance between the two strain gauges and partly because of possible relative movement between the two surfaces. In Section 3.6 it was pointed out that, for the purpose of the numerical model, it was assumed that no relative movement between the barrel and wrapper is possible i.e. a fixed contact surface. In practice some relative movement might occur as the wrapper is only welded to the barrel around its edges and where the two halves of the wrapper meet. The external and internal strain gauges were to provide some validation on the fixed contact assumption. The outcome of the test is described in Section 4.6. 


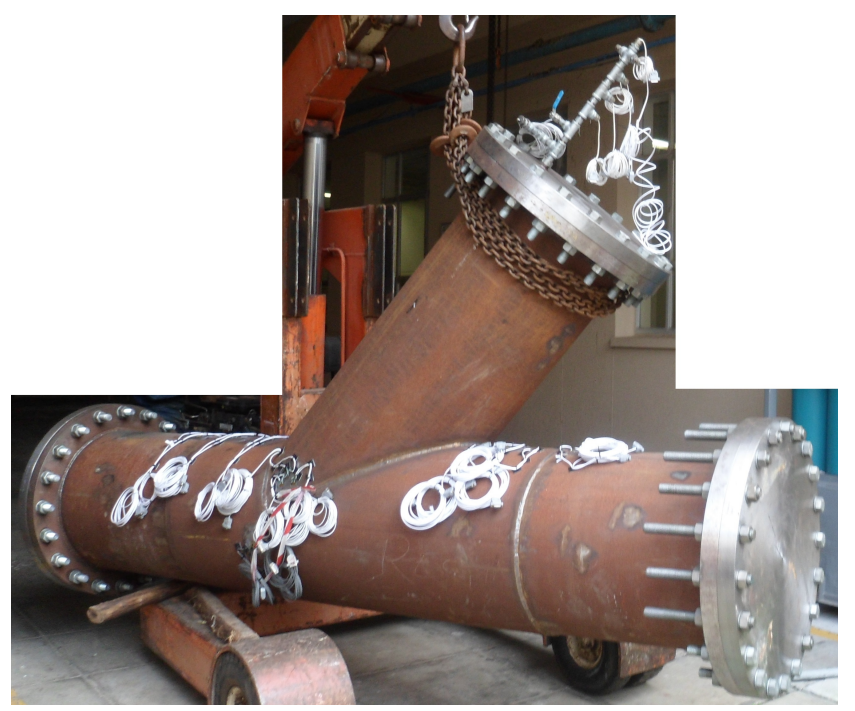

Figure 4.3: Test piece.

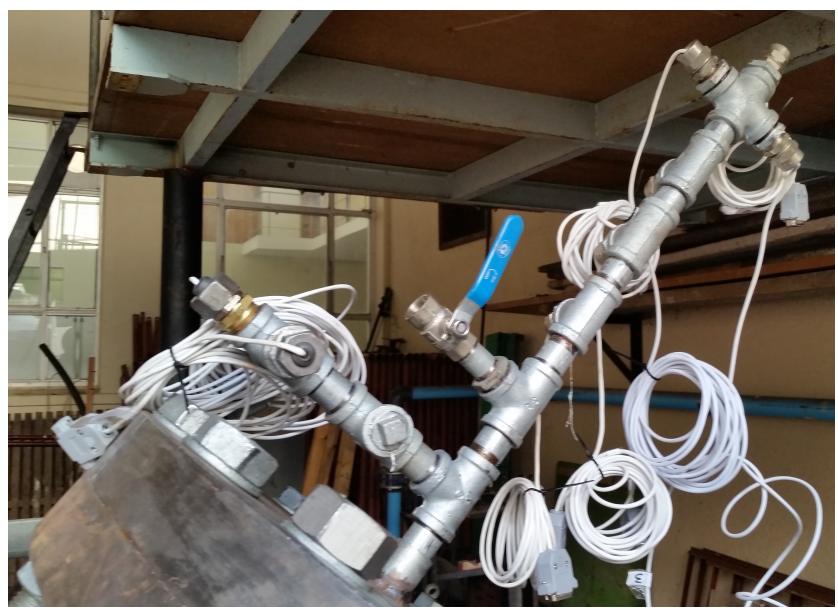

Figure 4.4: Sealing of internal strain gauge cables.

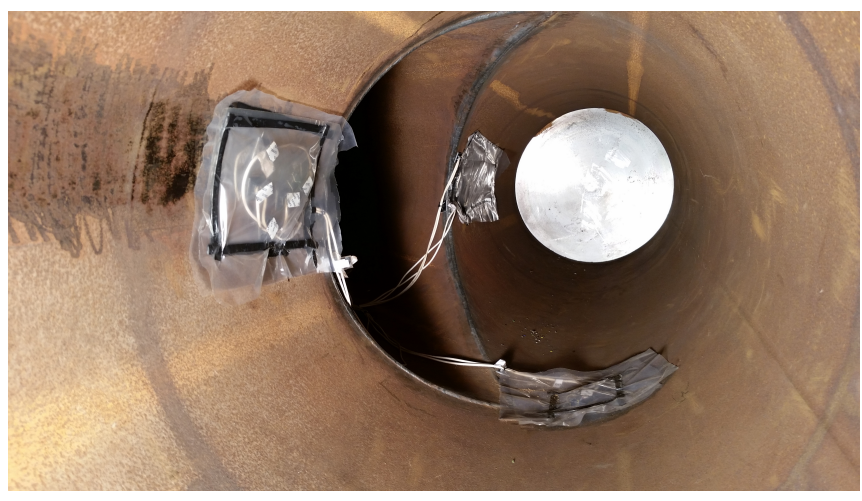

(a) Strain gauges on internal surfaces of special with blank flange on barrel end visible.

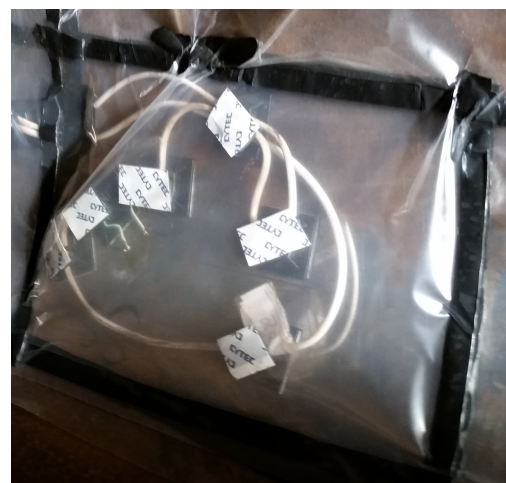

(b) Waterproof sealing over strain gauges.

Figure 4.5: Internal strain gauges with waterproof sealing. 
The strain gauges were placed in areas where high stresses and large displacements were expected as indicated by the numerical model. Figure 4.6 shows the strain gauge positions and orientations. The strain gauges that were finally recorded are shown in green and marked alphabetically. Table 4.2 provides a summary of the strain gauge orientations and Figure 4.7 shows the physical setup. The strain gauges had to be placed far enough away from welds not to be affected by the heat affected zones. The rule of thumb is widely believed to be twice the width of the weld. In this study, however, the strain gauges were placed further away as the objective was not to determine strains very close to the welds, but rather to validate the numerical model by comparison of a number of recorded strains with simulated strains.

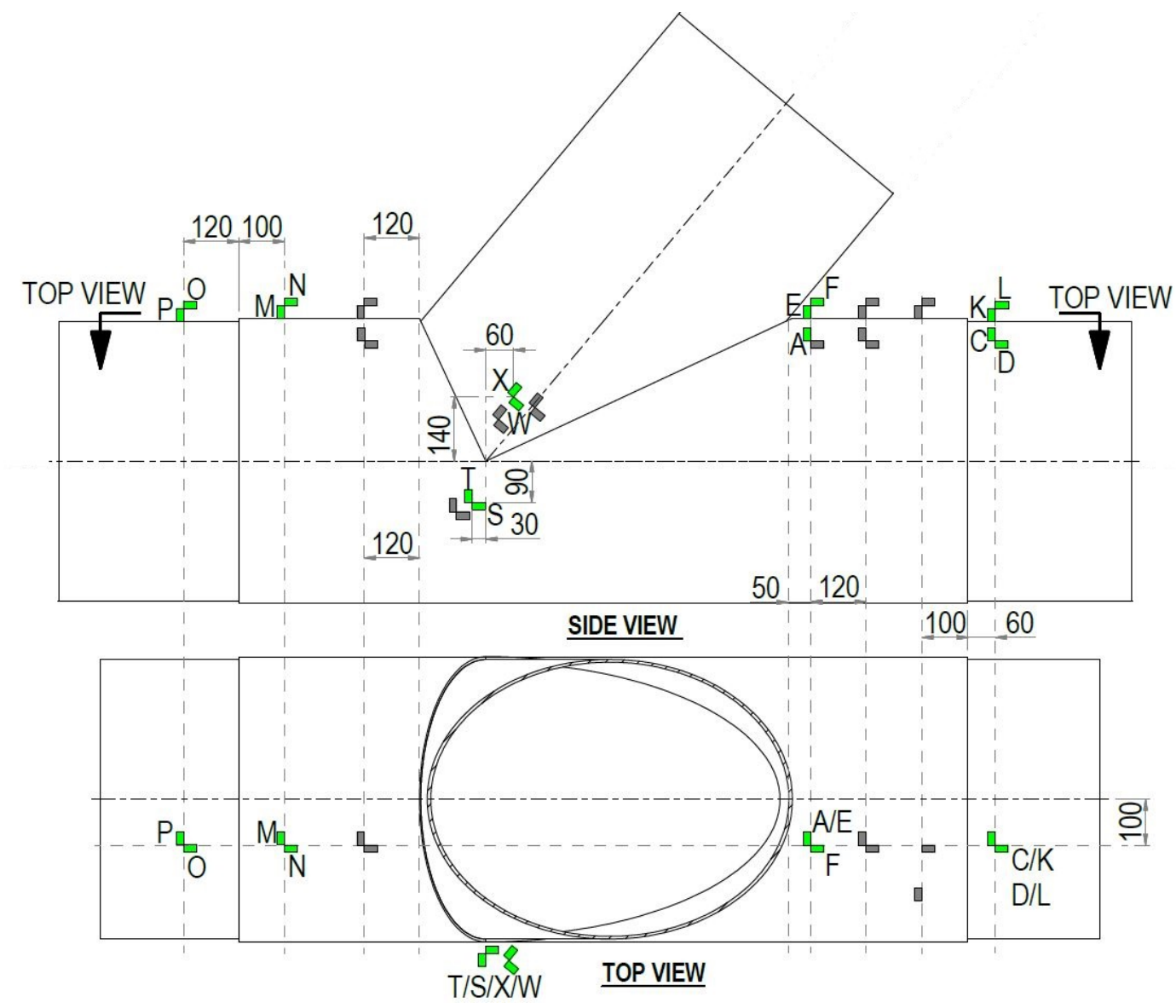

Figure 4.6: Strain gauge locations. 
Table 4.2: Orientation of strain gauges.

\begin{tabular}{ll}
\hline$A$ & Barrel hoop \\
$C$ & Barrel hoop \\
$D$ & Barrel longitudinal \\
$E$ & Barrel hoop \\
$F$ & Barrel longitudinal \\
$K$ & Barrel hoop \\
$L$ & Barrel longitudinal \\
$M$ & Barrel hoop \\
$N$ & Barrel longitudinal \\
$O$ & Barrel longitudinal \\
$P$ & Barrel hoop \\
$S$ & Barrel longitudinal \\
$T$ & Barrel hoop \\
$W$ & Branch hoop \\
$X$ & Branch longitudinal \\
\hline
\end{tabular}

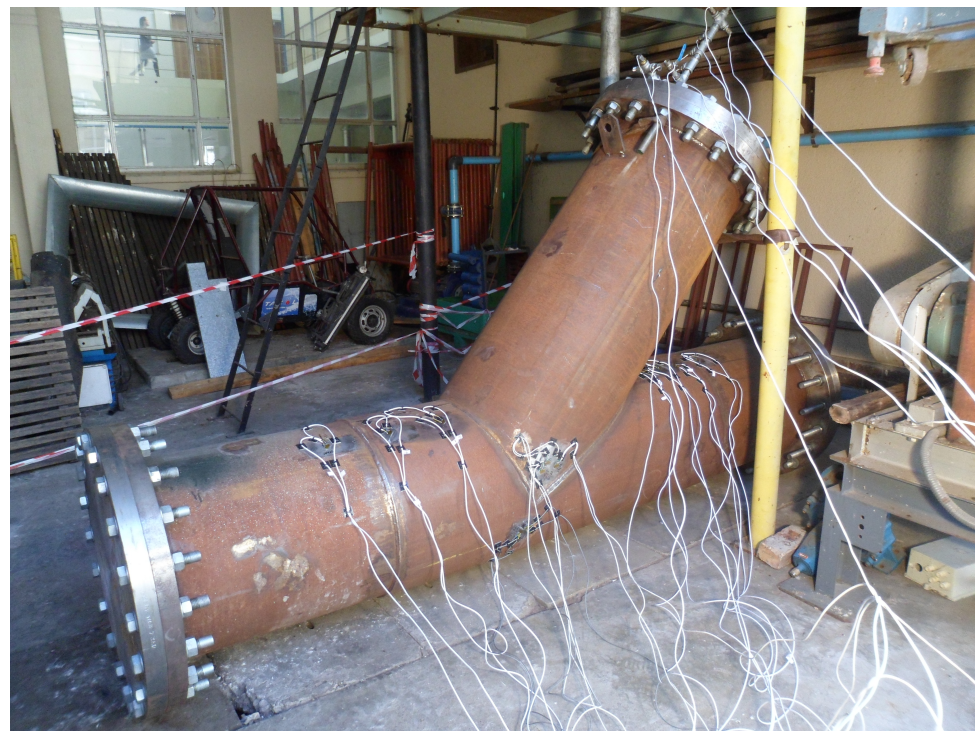

Figure 4.7: Test piece with strain gauges and cables.

\subsection{Tensile Testing}

Tensile tests were performed on five tensile specimens to determine the stressstrain relationships of the pipe material. Digital Imagery Correlation (DIC) technology was used to determine the Poisson's ratio. In order to determine the Poisson's ratio the longitudinal strains as well as the smaller lateral strains must be measured during a uniaxial tensile test. The longitudinal strains can be measured with an extensometer, but measuring the smaller lateral strains with strain gauges is problematic due to the small size of the tensile test 
specimen. DIC is a full-field image analysis method, based on grey value digital images that can determine the contour and displacements of an object and is thus a useful way of determining the lateral and longitudinal strains. Pre-test tensile samples are treated with a speckled pattern by spraying white and grey paint onto the surface. The first (reference) image is captured with no strain on the sample. The image is then split into small subsets and the patterns within each subset of subsequent images are compared to the reference image. The displacements are then calculated from this and a strain/displacement map is produced (Bailey, 2014). The tensile test setup is shown in Figure 4.8 with a typical tensile sample undergoing necking. It can be seen that the speckled surface is compromised by the high strains experienced after yielding. The experimentally obtained stress-strain curve is shown in Figure 4.9. The special was modelled with elastic perfectly plastic material behaviour. It can, however, be seen from Figure 4.9 that the pipe material does not behave elastic perfectly plastic and this affects the failure point of the special. The validity of assuming linear elastic plastic behaviour in FE analyses performed in this study is explained in Section 3.7.1. The actual yield strength of the test piece was found to be $410 \mathrm{MPa}$ and the Young's modulus was calculated as $210 \mathrm{GPa}$ using the average of three of the tests. The Poisson's ratio converged to approximately 0.28 as shown in Figure 4.10.

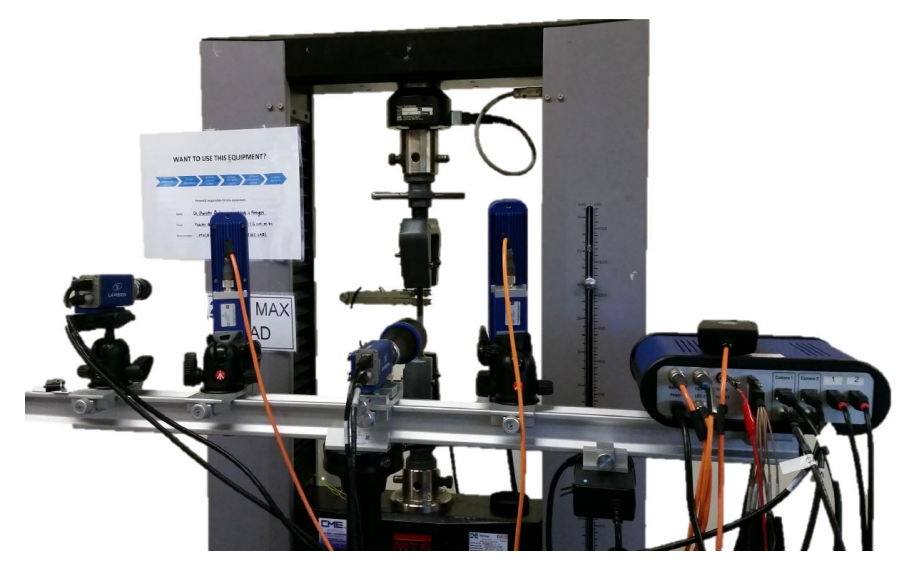

(a) DIC and tensile testing equipment.

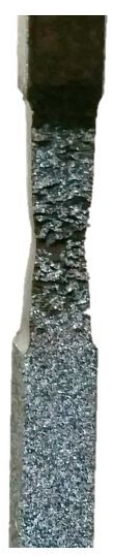

(b) Test sample with speckled surface for DIC image analysis.

Figure 4.8: Typical tensile test setup. 


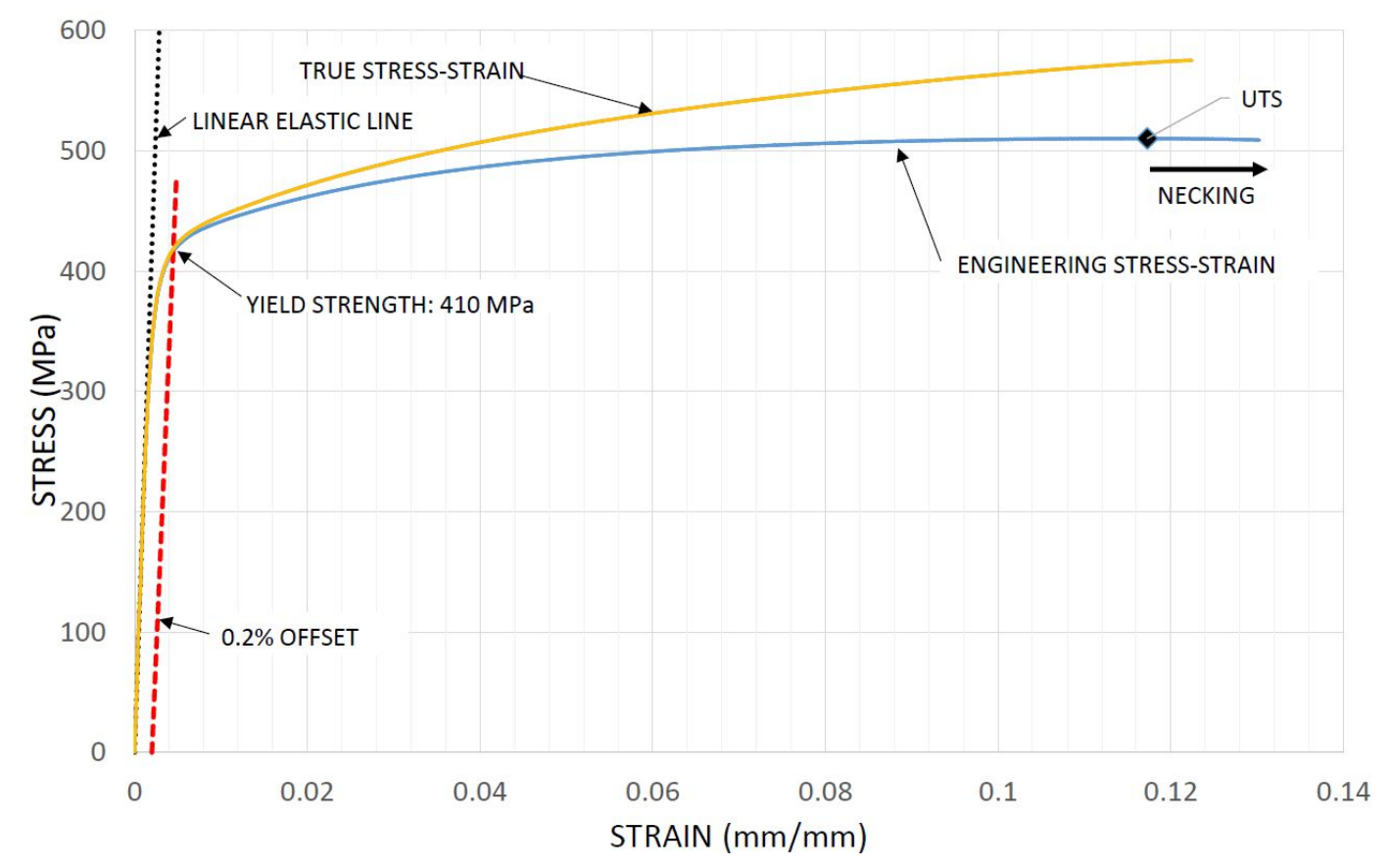

Figure 4.9: Stress-strain curve of test piece material.

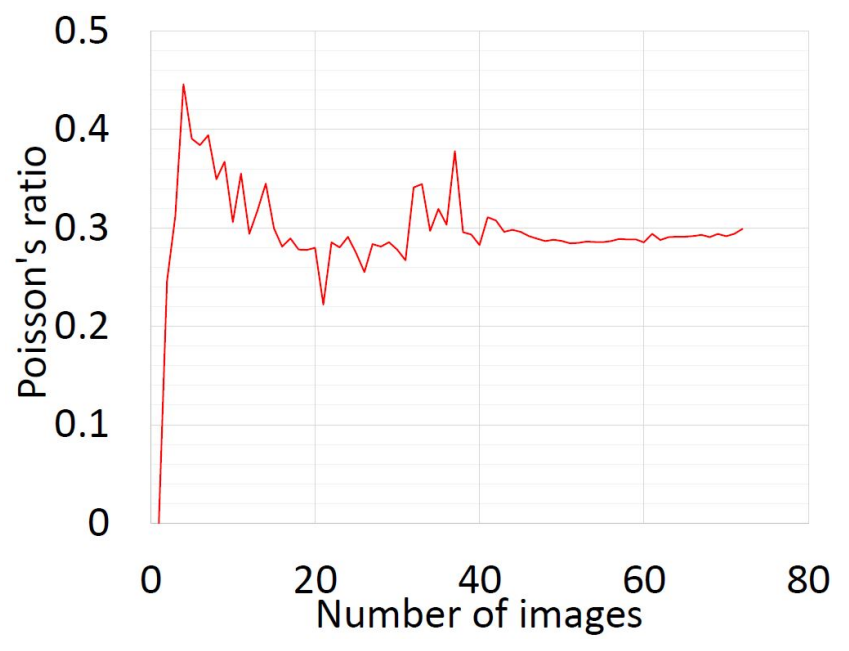

Figure 4.10: Convergence of Poisson's ratio.

\subsection{Experimental Procedure}

The special was pressurised with a positive displacement type high pressure electric pump. Water was used to pressurise the test piece and it was filled through a small valve connected to the nipple on the blank flange bolted to the branch end. A small piping assembly was bolted to the blank flange nipple. Cables for the internal strain gauges were taken through this piping assembly.

A number of tests were performed prior to the failure test to determine the condition of strain gauges, ensure proper cable connections and achieve the 
best possible setup for the failure test. During the initial tests it was found that the strain gauges react instantaneously to a change in internal pressure whereas there is a delay with the pressure transmitter upon a sudden internal pressure change. In order to account for this delay the damping time on the pressure transmitter was reduced and the special was pressurised slowly by controlling the flow rate from the pump to the special. Initial tests showed that a number of the strain gauges have failed, possibly because of failure of the adhesive used to secure it to the substrate. These strain gauges could be seen to have lifted off from the steel substrate at certain points on the strain gauge. This was expected to happen and therefore additional strain gauges were secured during the experimental setup to allow for some redundancy. The strain gauges that showed the most sensible and reliable output during initial tests (refer Table 4.2) were recorded during the pre-failure and failure tests. The most sensible and reliable strain gauges were considered to be the ones that corresponded well with the pressure in a normalised fashion and where no sudden changes in strain magnitudes were present. The camera that recorded the acute corner showed a significant amount of deformation with the branch and barrel bulging around the welded interface. Due to permanent deformation during initial trial tests sensible quantitative deformations could not be obtained. However, photos of the acute corner taken prior to, during and after the pressure tests are shown in Figure 3.19b with visible permanent deformation.

During initial trial tests and prior to recording of strain gauges the test piece was pressurised to approximately $2.9 \mathrm{MPa}$. As a result the test piece material underwent yielding at a number of strain gauge locations. This is discussed further in the next section.

\subsection{Discussion of Experimental Results}

Initial trial tests to an internal pressure of $2.9 \mathrm{MPa}$ affected the subsequent strain recordings of strain gauges where material yielding occurred during initial trial tests. The strains were not recorded during the initial trial tests due to failure of the data acquisition equipment. The damage was caused by incorrect wiring of the extension lead provided to power the equipment. This caused damaged to the electronic circuit board of the strain gauge amplifier. Strains were only recorded thereafter and prior to the failure test. The special was pressurised to the same internal pressure as during initial trial tests. Therefore, the recorded strains represent only the elastic strains of the reloading case as indicated by the reloading region on the stress-strain curve in Figure 4.11.

Manufacturing of the test piece and the procurement of strain gauges have significant financial and time implications. Hence, it was not feasible to set up a new test piece model which has not undergone any material yielding. Instead, recorded strains were analysed and the observations and conclusions are 
presented. Comparisons between recorded and simulated strains are discussed in Chapter 5.

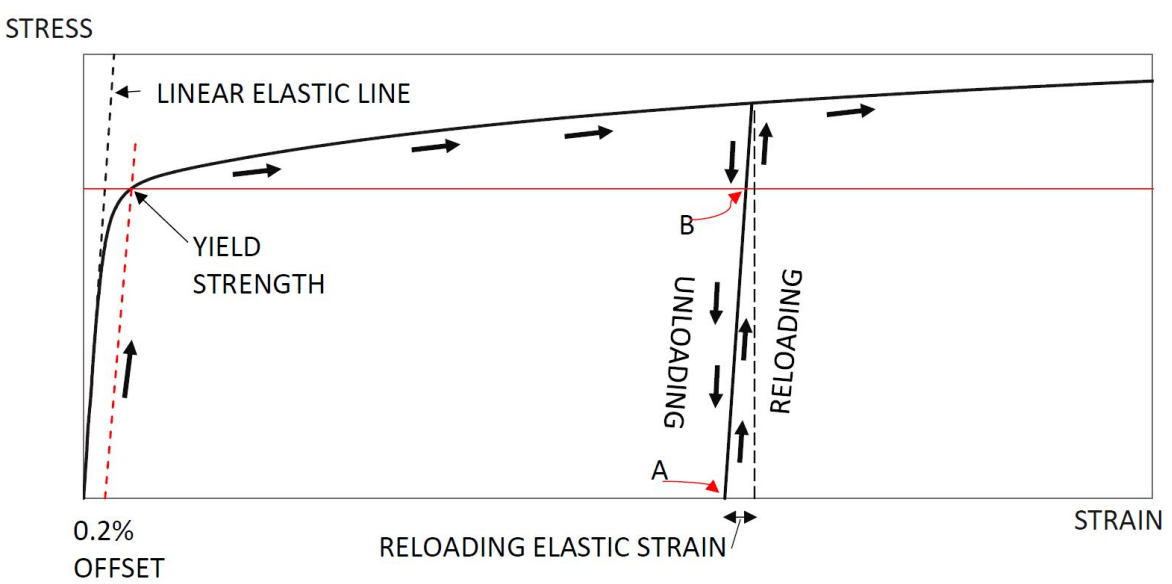

Figure 4.11: Typical unloading/reloading curve with reloading elastic strain.

Typical recorded pressure and strain readings are shown in Figure 4.12. In Figure 4.6 it was shown that strain gauge "E" is located in the crotch corner and is oriented in the hoop direction of the barrel. The negative strain values in Figure 4.12b indicate that this strain gauge is in compression. Curves of other strain gauges listed in Table 4.2 are shown in Appendix B. Figure 4.13a and Figure 4.13b shows the internal pressure vs. strain for strain gauges "E" and "L". The difference in the loading and unloading curve is a classic case of mechanical hysteresis. The strains recorded with strain gauges "L" and "M" were normalised and, together with the normalised pressure, plotted in Figure 4.14. It can be seen that the pressure transducer reacted quickly with a change in internal pressure as is the case for strain gauges.

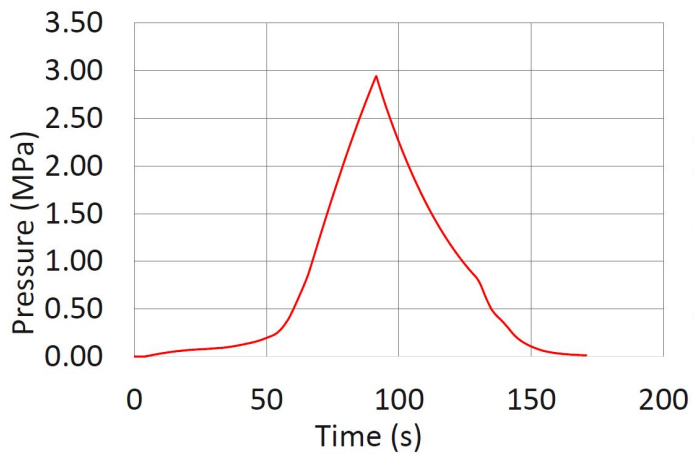

(a) Internal pressure.

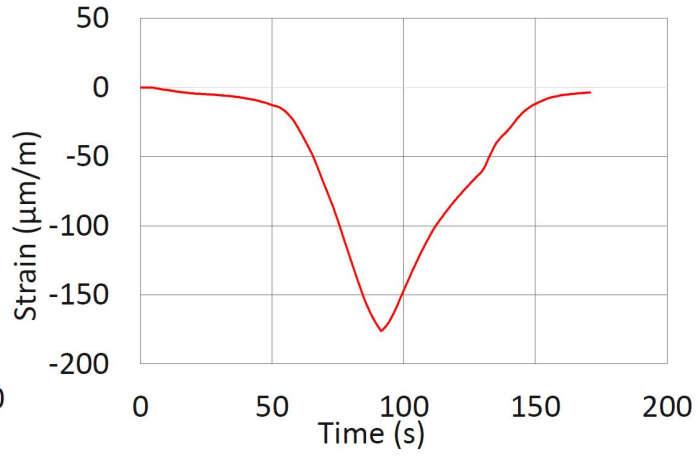

(b) Strain gauge "E".

Figure 4.12: Typical pressure and strain recordings. 


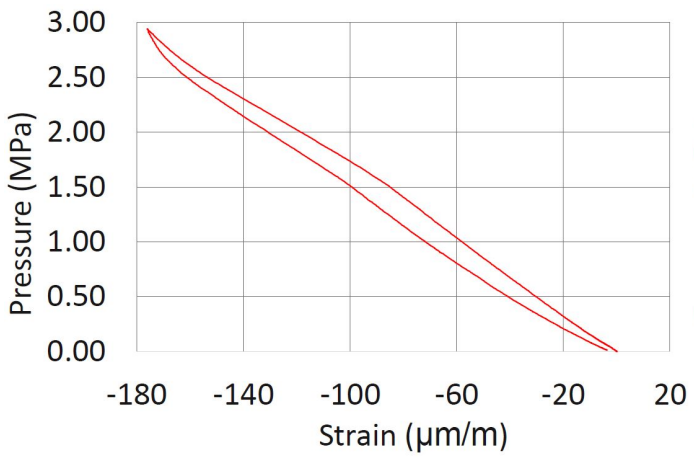

(a) Strain gauge "E".

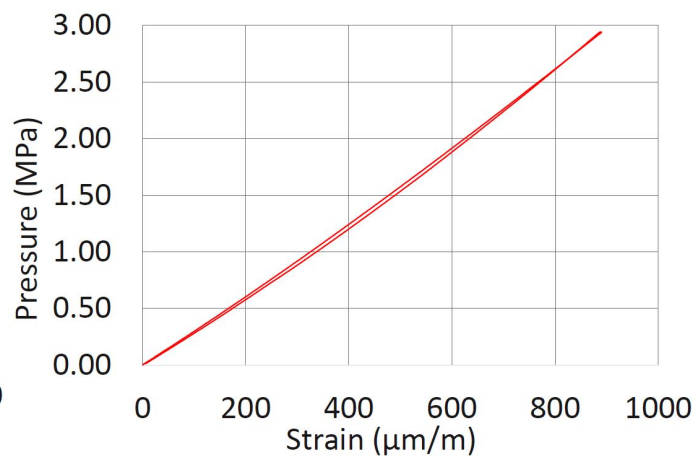

(b) Strain gauge "L".

Figure 4.13: Pressure vs. strain plot.

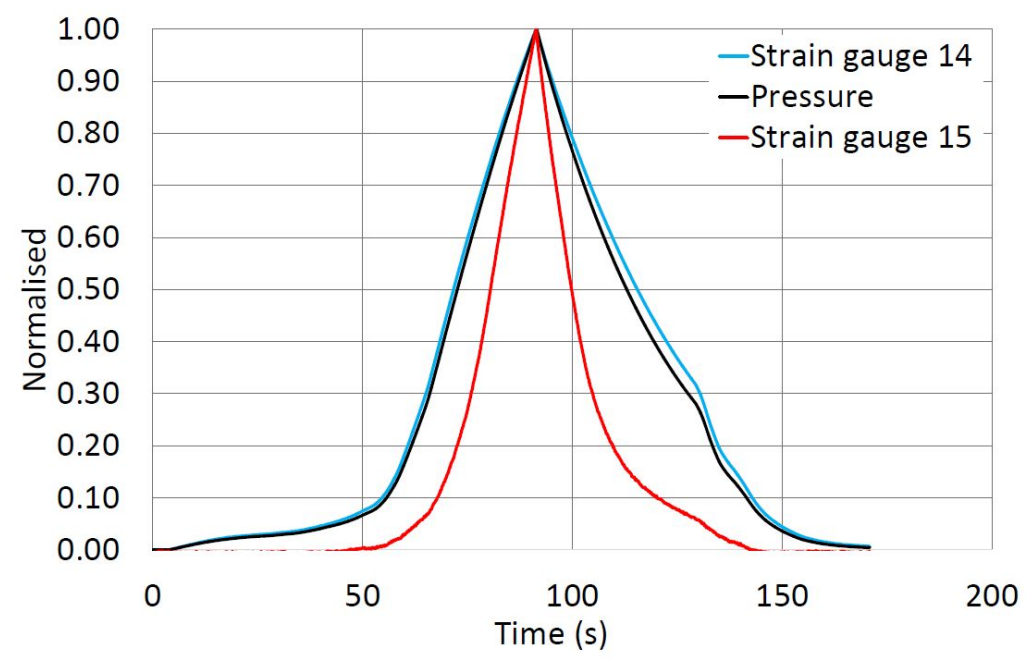

Figure 4.14: Normalised pressure and strain curves.

Most internal strain gauges failed, except for strain gauges "A", "C" and "D", presumably due to water ingress and water force damage to soldered connections. Resistance measurements over the strain gauge cables recorded did not match the resistance rating of the strain gauges used. Water could be seen leaking through the inner core of the cables connected to the internal strain gauges. This shows that water leaked through the waterproof seal shown in Figure 4.5b and was pressurised through the cables to where it was connected at the data acquisition equipment. Strain gauges "A" and "E" (absolute values) are plotted in Figure 4.15 to show the comparison in strains at an internal pressure of $2.98 \mathrm{MPa}$. It can be seen that significantly larger strains were measured by strain gauge "A". This is due to the combined thickness of the barrel and wrapper plates between the two strain gauges. The inside surface of the barrel at this point is in tension (positive strain values for strain gauge "A"), while the corresponding wrapper outer surface is in compression. Figure 4.16 shows a similar curve for strain gauge " $\mathrm{D}$ " and strain gauge "L", 
both in tension, with a higher strain measured by strain gauge "L". This is expected as the latter is on the outer surface of the barrel and strain gauge "D" is on the inner surface of the barrel. The normal distance between these two strain gauges is the thickness of the barrel only. As expected the difference in strain readings increases with an increase in normal distance (i.e. thicker plates) between two strain gauges measuring hoop strains or where plate bending occurs.

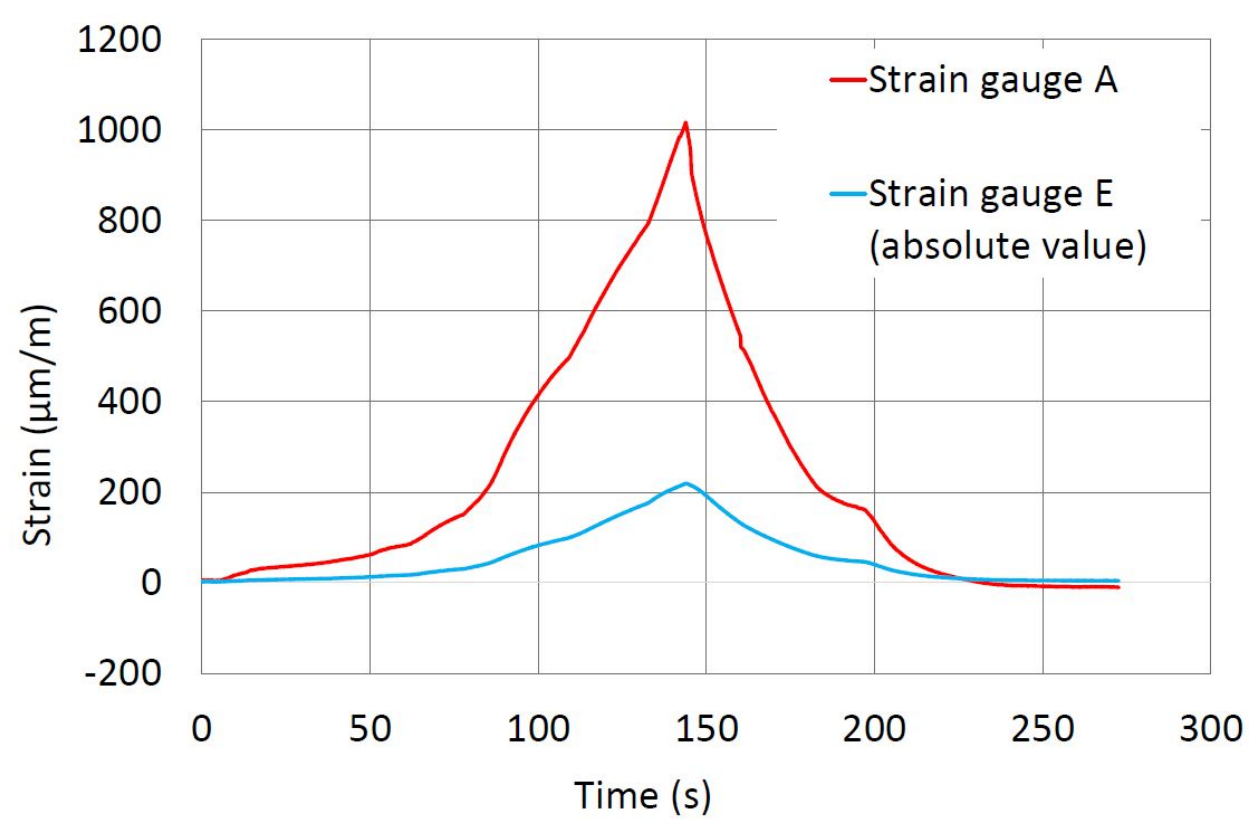

Figure 4.15: Strain gauges "A" (red) and "E" (blue). 


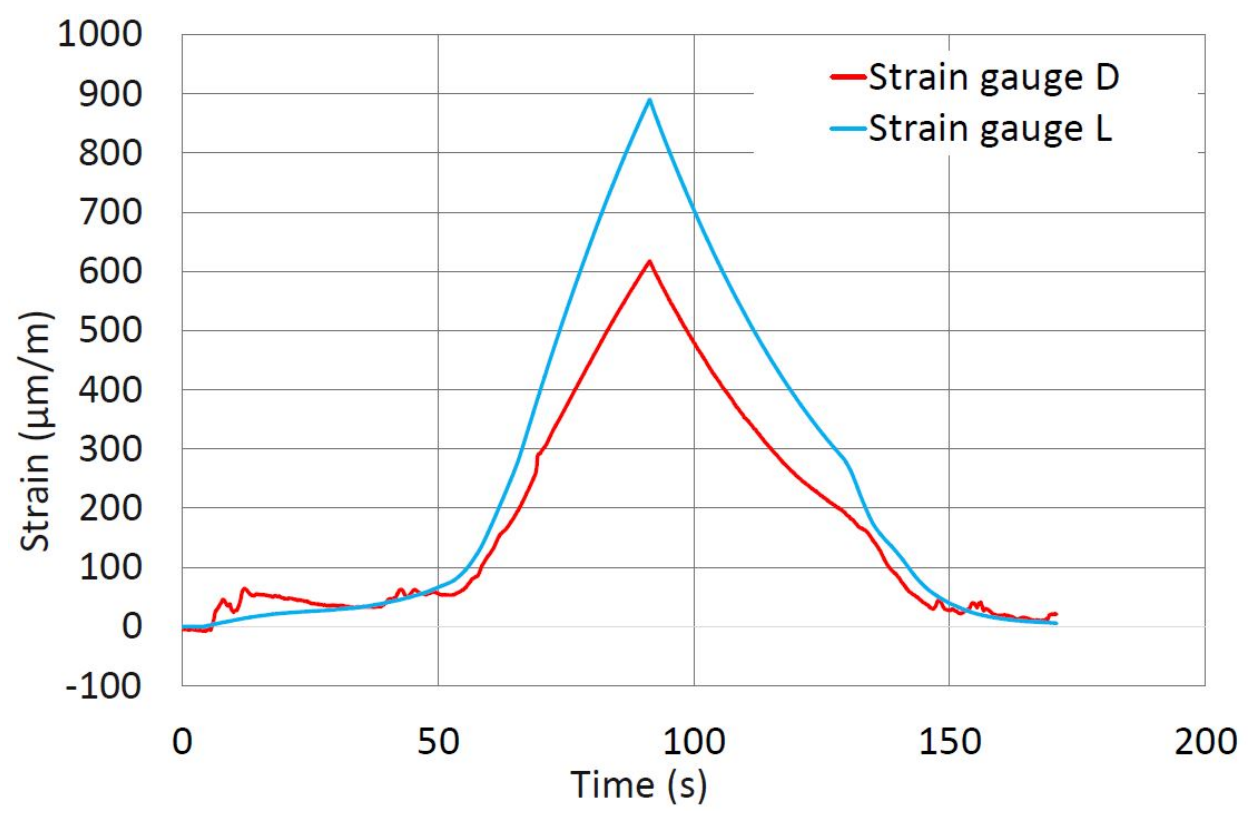

Figure 4.16: Strain gauges "D" (red) and "L" (blue).

The camera that recorded the acute corner showed a significant amount of deformation with the branch and barrel bulging around the welded interface, as shown in Figure 4.17. Lines tangent to the branch and wrapper outside surfaces are shown to distinguish deformations from the original shape of the test piece. It can be seen that some deformation recovered during depressurisation.

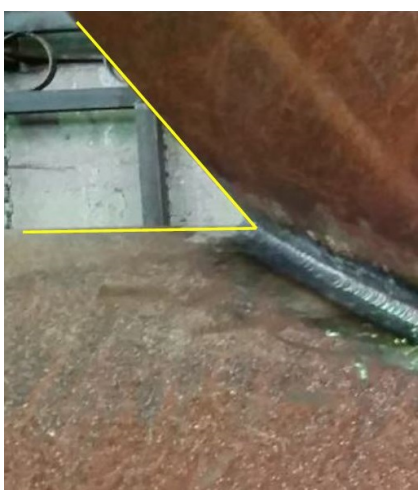

(a) Prior to pressure test.

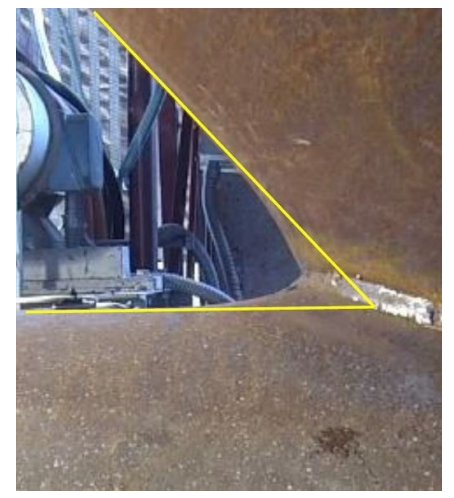

(b) At approximately 4.4 MPa.

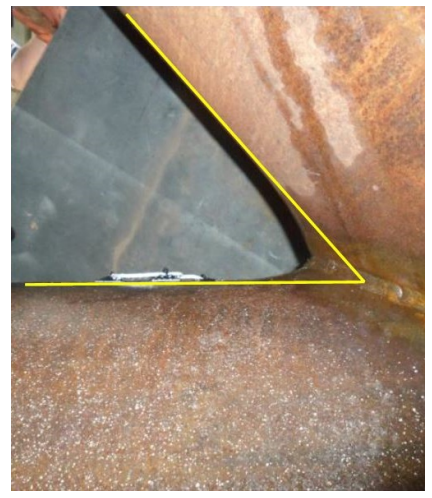

(c) Permanent deformation after pressure test.

Figure 4.17: Deformation of test piece acute corner. 


\section{Chapter 5}

\section{Validation of the Numerical Model}

\subsection{General}

The numerical model is to provide a tool with which new designs can be investigated and developed. Validation of the FE model is performed to provide some indication on the accuracy of assumptions made, boundary conditions chosen and procedures followed in the development of all FE models in this study. First a visual comparison was made between the physical and simulated model. Secondly, strain recordings from the data acquisition unit was compared with corresponding points in a representative model. If the relative difference between simulated and recorded elastic strains are within $20 \%$ then the model is assumed to be successfully validated.

\subsection{Visual Comparison}

High definition cameras and mechanical dial gauges confirmed, in a qualitative manner, that the largest deformations typically occur at the outside edge of the barrel/branch intersection, indicated by point "A" on Figure 3.19b, page 49. Useful quantitative measurements could not be obtained due to the fact that permanent deformation occurred during the initial trial test as explained in Section 4.6. However, the tee did not fail at the simulated failure load of 3.2 MPa. Figure 5.1 shows the stress distribution at the simulated failure load, assuming elastic perfectly plastic behaviour and a yield strength of $410 \mathrm{MPa}$.

After the internal pressure was released a small crack could be seen in the acute corner, as shown in Figure 5.2. As mentioned in Section 4.2 all welds were visually inspected for cracks prior to the hydrostatic test and none were found. Sang et al. (2002) showed that 90 degree specials typically fail in the same region with the propogation of a crack in the longitudinal direction of the branch and barrel. In this study it appears that the crack originates from a minor welding defect in the form of a pinhole. It is expected that the test piece will fail here as a result of crack propagation, considering that the 
highest stresses occur in the acute corner, as shown in Figure 5.1. Important to note here is that welding defects are quite common and largely attributed to human error. This despite modern testing techniques such as X-rays and dye-pen testing. The defect shown in Figure 5.2 is therefore not an exception and it emphasizes the need for a sufficient factor of safety and testing of welds. Only ductile failure is considered in this study and safety factors calculated accordingly.

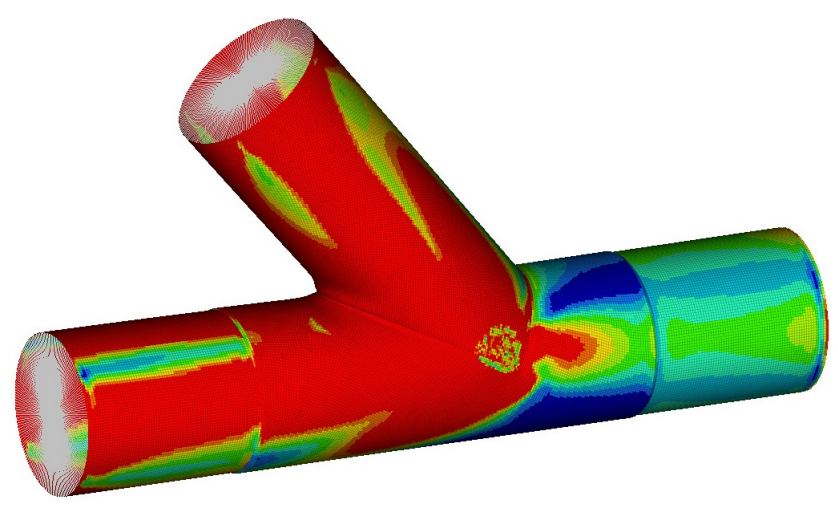

Figure 5.1: Elastic perfectly plastic behaviour (3.2 MPa).
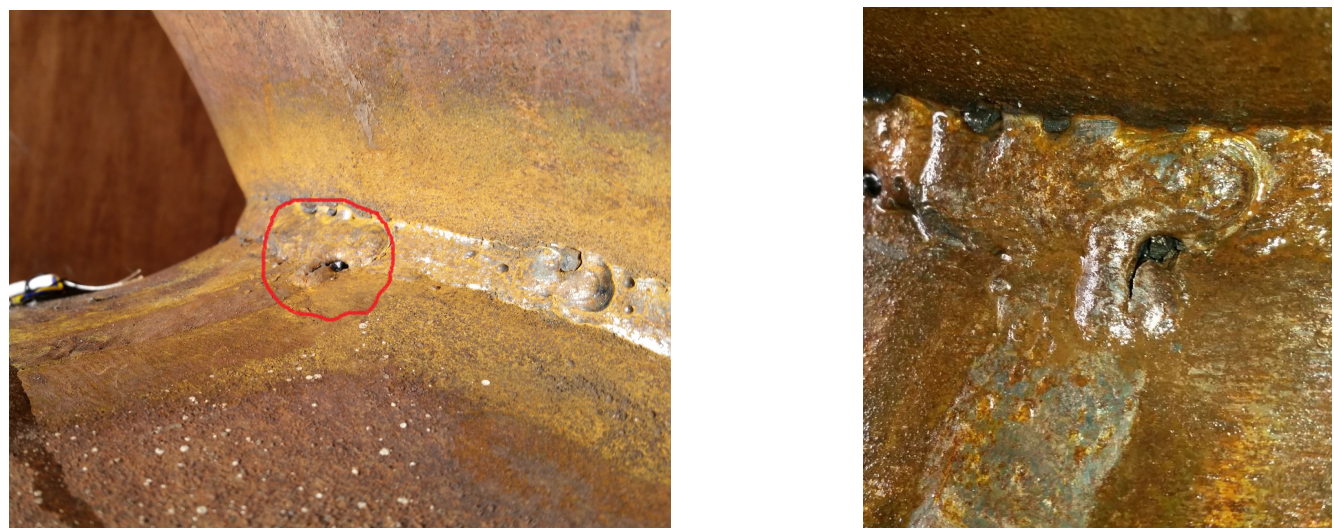

Figure 5.2: Small crack in acute corner. 


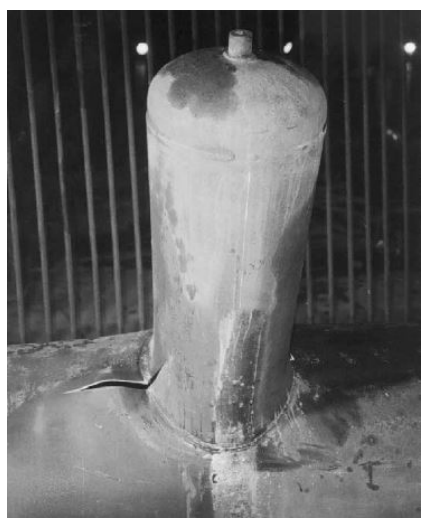

Figure 5.3: Typical failure of 90 degree special (Sang et al. (2002)).

The deformation in the acute corner of the test piece compares, in a qualitative manner, well with the simulated deformation shown in Figure 5.4. Lines approximately tangent to the branch and wrapper outside surfaces are shown to distinguish deformations from the undeformed shape. Slight bulging of the branch, indicated by the arrow, is observed around the welded interface. In this study it is concluded that this is a result of element overlapping (shown in Figures 3.14 and 3.15 on page 45 ) and the extent of the contact surface defined between the barrel and wrapper. Both of these factors provide some additional stiffness to the intersection.

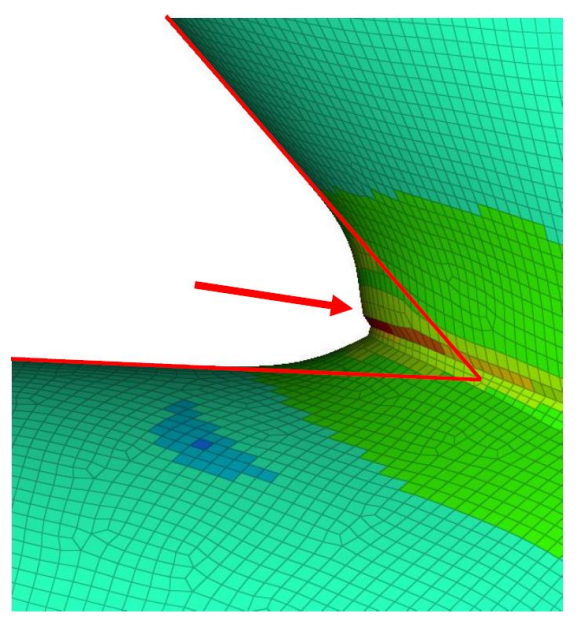

Figure 5.4: Simulated deformation in acute corner.

\subsection{Numerical Comparison}

The approximate locations of the external recorded strain gauges are shown in Figure 5.5. The simulated elastic strains for each strain location are presented 
in Tables 5.1 and 5.2 for two different internal pressure loads. Physically measured reloading elastic strains (see Figure 4.11 on page 77 ) are also shown for the corresponding strain gauges. Physical measurements and simulated results that compare well (within 20\%) are shown in bold. The relative differences are calculated using the measured strains as reference.

The simulated elemental strain corresponding to strain gauge "W" does not compare well (within 20\%) with the measured strain for this strain gauge at an internal pressure load of $2 \mathrm{MPa}$. This is largely attributed to the fact that strain gauge "W" is located at the transition from positive to negative hoop strains, as shown in Figure 5.6. The negative strain measured by gauge "E" at both load conditions is supported by the negative hoop strain of the corresponding element as shown in Figure 5.7a on page 88. Under an internal pressure load the shape of the cross section shown in Figure 2.5 (page 9) is forced into a circular shape. This results in high stresses and negative barrel hoop strains in the acute corner. In Table 5.2 it can be seen that at an internal pressure load of $2 \mathrm{MPa}$ the simulated and measured strains for strain gauges "L" and "O" compare better than at an internal pressure load of $1 \mathrm{MPa}$. However, the measured and recorded strains for strain gauges " $\mathrm{N}$ " and "W" compare less favourable. The difference between the simulated and measured strains for strain gauge "A" could be attributed to the contact surface modelled between the wrapper and barrel. Similar to strain gauge "W", at an internal pressure load of $2 \mathrm{MPa}$, the discontinuous strain contours in the vicinity of strain gauges "A", "D", "F", "K", "M", "N", "S" and "W" together with the sensitivity in selecting the applicable element seems to be the main reasons for the difference between measured and simulated strains in Table 5.2. The same conclusions applies for the results in Table 5.1. Also, the smaller the strains are the more sensitive it is to differences.

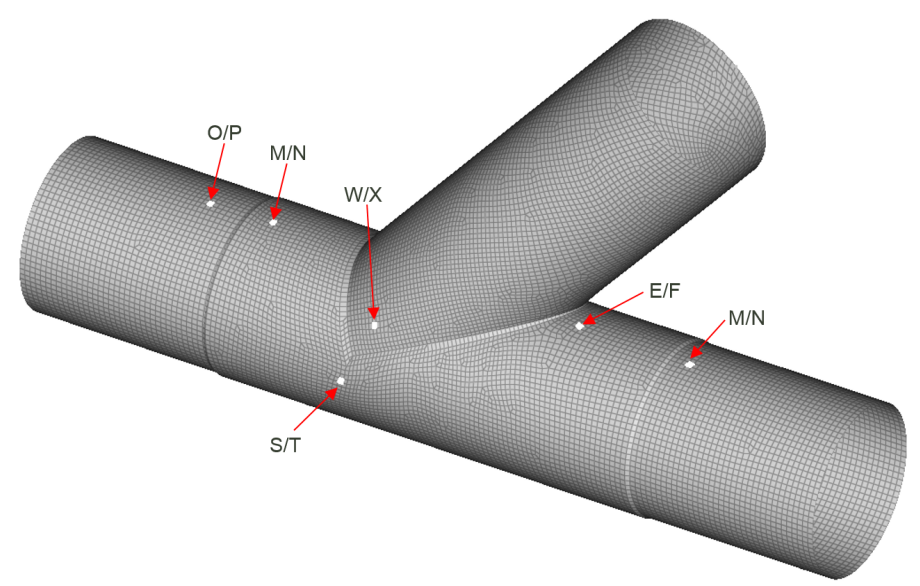

Figure 5.5: Approximate strain gauge locations. 
Table 5.1: Comparison between simulated and recorded strains at an internal pressure load of $1 \mathrm{MPa}$ with strains measured in $\mu \mathrm{m} / \mathrm{m}$.

\begin{tabular}{l|cc|rr} 
Strain gauge & Measured & Simulated & Abs Difference & Rel Difference (\%) \\
\hline$A$ & 222 & 360 & 138 & 62.0 \\
$C$ & $\mathbf{1 5 9}$ & $\mathbf{1 4 3}$ & $\mathbf{- 1 6}$ & $\mathbf{- 1 0}$ \\
$D$ & $\mathbf{2 3 3}$ & $\mathbf{2 2 5}$ & $\mathbf{- 8}$ & $\mathbf{- 3 . 4}$ \\
$E$ & -83 & -57 & 26 & 31.3 \\
$F$ & -110 & 80 & 190 & 172 \\
$K$ & 152 & 210 & -58 & 38.1 \\
$L$ & 258 & 325 & 67 & 25.9 \\
$M$ & 18 & 9 & -9 & -50.0 \\
$N$ & $\mathbf{8 5}$ & $\mathbf{1 0 2}$ & $\mathbf{1 7}$ & $\mathbf{2 0 . 0}$ \\
$O$ & 158 & 196 & 38 & 24.0 \\
$P$ & 153 & 186 & 33 & 21.5 \\
$S$ & 65 & -16 & -81 & 124.6 \\
$T$ & $\mathbf{1 5 4}$ & $\mathbf{1 2 6}$ & $\mathbf{2 8}$ & $\mathbf{1 8 . 2}$ \\
$W$ & $\mathbf{6 9}$ & $\mathbf{5 7}$ & $\mathbf{1 2}$ & $\mathbf{1 7 . 4}$ \\
$X$ & $\mathbf{2 3 7}$ & $\mathbf{2 4 2}$ & $\mathbf{5}$ & $\mathbf{2 . 1}$ \\
\hline
\end{tabular}

Table 5.2: Comparison between simulated and recorded strains at an internal pressure load of $2 \mathrm{MPa}$ with strains measured in $\mu \mathrm{m} / \mathrm{m}$.

\begin{tabular}{l|cc|rr} 
Strain gauge & Measured & Simulated & Abs Difference & Rel Difference $(\%)$ \\
\hline$A$ & 853 & 682 & 171 & 20.0 \\
$C$ & $\mathbf{3 2 5}$ & $\mathbf{3 0 8}$ & $\mathbf{- 1 7}$ & $\mathbf{- 5 . 2}$ \\
$D$ & 551 & 432 & -119 & -21.6 \\
$E$ & $\mathbf{- 1 1 3}$ & $\mathbf{- 1 1 8}$ & $\mathbf{- 5}$ & $\mathbf{- 4 . 4}$ \\
$F$ & -973 & 129 & 1102 & 113.3 \\
$K$ & 242 & 420 & 121 & 57.6 \\
$L$ & $\mathbf{6 2 0}$ & $\mathbf{6 2 4}$ & $\mathbf{4}$ & $\mathbf{0 . 6}$ \\
$M$ & 8 & 42 & 34 & 425 \\
$N$ & 161 & 182 & 85 & 102 \\
$O$ & $\mathbf{3 2 0}$ & $\mathbf{3 9 6}$ & $\mathbf{2 1}$ & $\mathbf{6 . 6}$ \\
$P$ & $\mathbf{2 9 4}$ & $\mathbf{3 4 8}$ & $\mathbf{5 4}$ & $\mathbf{1 8 . 4}$ \\
$S$ & 73 & -21 & 94 & 128.8 \\
$T$ & $\mathbf{1 9 6}$ & $\mathbf{2 0 6}$ & $\mathbf{1 0}$ & $\mathbf{5 . 1}$ \\
$W$ & -322 & 71 & 393 & 122 \\
$X$ & $\mathbf{5 5 4}$ & $\mathbf{5 2 9}$ & $\mathbf{2 5}$ & $\mathbf{4 . 5}$ \\
\hline
\end{tabular}

To support this observation of sensitivity in the selection of the correct element corresponding to the actual measured strain gauge the simulated strains for elements in close proximity to the strain gauge locations, shown in Figure 4.6, were recorded. The elements with strains closest to the measured 
strains and within a radius of $20 \mathrm{~mm}$ of the approximate strain gauge locations were used for the recorded strains shown in Tables 5.3 and 5.4. It can be seen that the simulated and measured strains compare well for most strain gauges. This shows that the accuracy of simulated strains for comparison with measured strains are highly sensitive to the selection of the correct elements. The sensitivity exists even though mesh refinement was done and the model converged. It must be noted that the size and shape of the test piece complicates the exact measurement of strain gauge locations and the subsequent selection of corresponding elements. Special care was taken in this study to measure exact strain gauge locations from reference points and centerlines used by the manufacturer of the special. The use of specially designed jigs could have been used to confirm the accuracy of these measurements, but there was not sufficient time and money available for this. In future studies special attention should be paid to the selection of strain gauge locations with consideration to the possibility of discontinuous strain contours. An attempt should also be made to confirm the accuracy of strain gauge location measurements on the physical test piece. Strains gauges should also be placed in areas where higher strains are expected.

Table 5.3: Comparison between simulated strains for nearby elements and recorded strains at an internal pressure load of $1 \mathrm{MPa}$ with strains measured in $\mu \mathrm{m} / \mathrm{m}$. The simulated strains were recorded with elements in close proximity to the approximate strain gauge locations shown in Figure 4.6.

\begin{tabular}{l|cc|rr} 
Strain gauge & Measured & Simulated & Abs Difference & Rel Difference $(\%)$ \\
\hline$A$ & $\mathbf{2 2 2}$ & $\mathbf{1 8 7}$ & $\mathbf{- 3 5}$ & $\mathbf{- 1 5 . 7}$ \\
$C$ & $\mathbf{1 5 9}$ & $\mathbf{1 3 5}$ & $\mathbf{- 2 4}$ & $\mathbf{- 1 5 . 1}$ \\
$D$ & $\mathbf{2 3 3}$ & $\mathbf{1 9 9}$ & $\mathbf{- 3 4}$ & $\mathbf{- 1 4 . 5 9}$ \\
$E$ & $\mathbf{- 8 3}$ & $\mathbf{- 6 7}$ & $\mathbf{1 6}$ & $\mathbf{1 9 . 2}$ \\
$F$ & $\mathbf{- 1 1 0}$ & $\mathbf{- 8 9}$ & $\mathbf{2 1}$ & $\mathbf{1 9 . 9}$ \\
$K$ & $\mathbf{1 5 2}$ & $\mathbf{1 7 7}$ & $\mathbf{2 5}$ & $\mathbf{1 6 . 4}$ \\
$L$ & $\mathbf{2 5 8}$ & $\mathbf{2 3 2}$ & $\mathbf{- 2 6}$ & $\mathbf{- 1 0 . 0}$ \\
$M$ & 18 & 12 & -6 & -33.3 \\
$N$ & $\mathbf{8 5}$ & $\mathbf{7 7}$ & $\mathbf{- 8}$ & $\mathbf{- 9 . 4}$ \\
$O$ & $\mathbf{1 5 8}$ & $\mathbf{1 3 2}$ & $\mathbf{- 2 6}$ & $\mathbf{- 1 6 . 5}$ \\
$P$ & $\mathbf{1 5 3}$ & $\mathbf{1 4 1}$ & $\mathbf{- 1 2}$ & $\mathbf{- 7 . 8}$ \\
$S$ & $\mathbf{6 5}$ & $\mathbf{7 2}$ & $\mathbf{7 2}$ & $\mathbf{1 0 . 8}$ \\
$T$ & $\mathbf{1 5 4}$ & $\mathbf{1 6 9}$ & $\mathbf{1 5}$ & $\mathbf{9 . 7}$ \\
$W$ & 69 & 87 & 18 & 26.1 \\
$X$ & $\mathbf{2 3 7}$ & $\mathbf{2 1 5}$ & $\mathbf{- 2 2}$ & $\mathbf{- 9 . 3}$ \\
\hline
\end{tabular}


Table 5.4: Comparison between simulated strains for nearby elements and recorded strains at an internal pressure load of $2 \mathrm{MPa}$ with strains measured in $\mu \mathrm{m} / \mathrm{m}$. The simulated strains were recorded with elements in close proximity to the approximate strain gauge locations shown in Figure 4.6.

\begin{tabular}{l|cc|rr} 
Strain gauge & Measured & Simulated & Abs Difference & Rel Difference (\%) \\
\hline$A$ & $\mathbf{8 5 3}$ & $\mathbf{9 1 5}$ & $\mathbf{6 2}$ & $\mathbf{7 . 3}$ \\
$C$ & $\mathbf{3 2 5}$ & $\mathbf{3 4 5}$ & $\mathbf{2 0}$ & $\mathbf{6 . 2}$ \\
$D$ & $\mathbf{5 5 1}$ & $\mathbf{5 8 8}$ & $\mathbf{3 7}$ & $\mathbf{6 . 7}$ \\
$E$ & -113 & -80 & 33 & 29.2 \\
$F$ & $\mathbf{- 9 7 3}$ & $\mathbf{- 8 8 8}$ & $\mathbf{8 5}$ & $\mathbf{8 . 7}$ \\
$K$ & $\mathbf{2 4 2}$ & $\mathbf{2 7 4}$ & $\mathbf{3 2}$ & $\mathbf{1 3 . 2}$ \\
$L$ & $\mathbf{6 2 0}$ & $\mathbf{5 8 7}$ & $\mathbf{- 3 3}$ & $\mathbf{- 5 . 3}$ \\
$M$ & 8 & 2 & -6 & -75 \\
$N$ & $\mathbf{1 6 1}$ & $\mathbf{1 4 5}$ & $\mathbf{- 1 6}$ & $\mathbf{- 9 . 9}$ \\
$O$ & $\mathbf{3 2 0}$ & $\mathbf{3 0 5}$ & $\mathbf{- 1 5}$ & $\mathbf{- 4 . 7}$ \\
$P$ & $\mathbf{2 9 4}$ & $\mathbf{2 7 6}$ & $\mathbf{- 1 8}$ & $\mathbf{- 6 . 1}$ \\
$S$ & 73 & 45 & 28 & 38.3 \\
$T$ & $\mathbf{1 9 6}$ & $\mathbf{1 7 5}$ & $\mathbf{- 2 1}$ & $\mathbf{- 1 0 . 7}$ \\
$W$ & $\mathbf{- 3 2 2}$ & $\mathbf{- 2 5 9}$ & $\mathbf{6 3}$ & $\mathbf{1 9 . 6}$ \\
$X$ & $\mathbf{5 5 4}$ & $\mathbf{4 9 8}$ & $\mathbf{- 5 6}$ & $\mathbf{- 1 0 . 1}$ \\
\hline
\end{tabular}

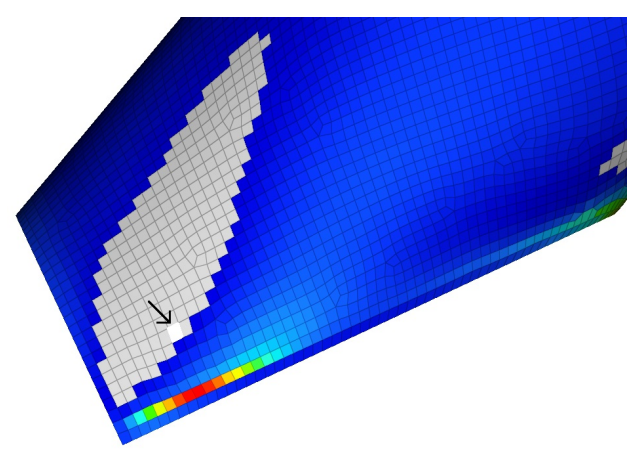

(a) Hoop strains.

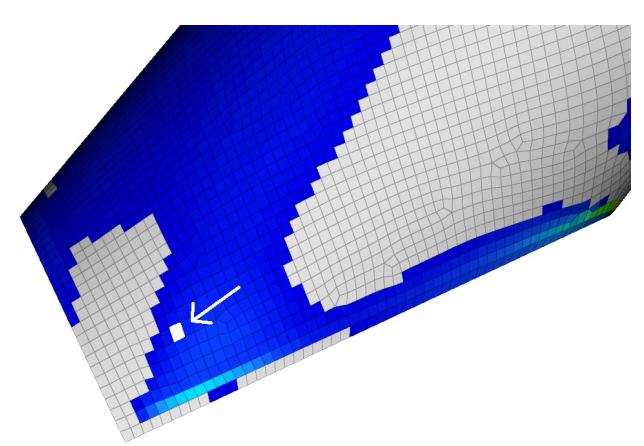

(b) Longitudinal strains.

Figure 5.6: Branch with negative longitudinal and hoop elemental strains on the external surface shown in grey. 


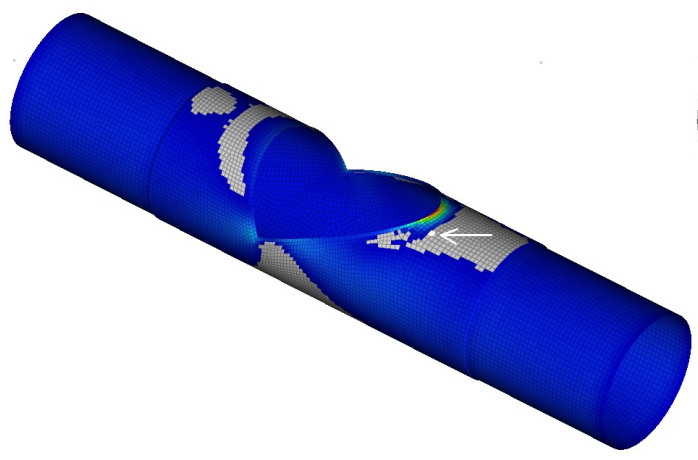

(a) Hoop strains.

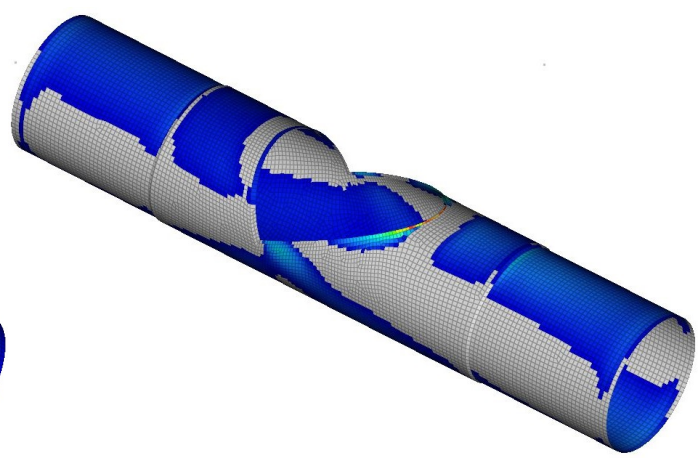

(b) Longitudinal strains.

Figure 5.7: Barrel and wrapper elements with negative longitudinal and hoop elemental strains on external surfaces shown in grey.

In Section 4.6 it was pointed out that there is a difference in strain magnitudes between internal and external surfaces. Table 5.5 provides a summary of strains for these internal and external strain gauges. The simulated results show that there is significant difference between strain gauge "A" and "E" as well as "D" and "L" except for the simulated strain between strain gauges "D" and "L". Incidentally, the selection of elements corresponding to the measured strain gauges also affects these results.

Table 5.5: Strain comparison for internal and external surfaces at an internal pressure load of $2 \mathrm{MPa}$, measured in $\mu \mathrm{m} / \mathrm{m}$.

\begin{tabular}{l|cc|rr} 
Strain gauge & Simulated & Measured & Abs Difference & Rel Difference \\
\hline$A$ & 853 & 682 & -171 & -20.0 \\
$E$ & -113 & -118 & -5 & -4.4 \\
Absolute Difference & -966 & -800 & & \\
Relative Difference & -113.2 & -117.3 & & -21.6 \\
\hline$D$ & $\mathbf{5 5 1}$ & 432 & -119 & 0.6 \\
$L$ & $\mathbf{6 2 0}$ & 624 & 4 & \\
Absolute Difference & $\mathbf{6 9}$ & 192 & & \\
Relative Difference & $\mathbf{1 2 . 5}$ & 44.4 & & \\
\hline
\end{tabular}

\subsection{Summary of Numerical Model Validation}

It can be concluded that the simulated model compares well with the physical data in a quantitative manner. The simulated elastic strains generally compare well (within 20\%) with the measured reloading elastic strains when elements with a simulated strain closest to the measured strain and in the close vicinity of that strain gauge is used as shown in Tables 5.3 and 5.4. The 
test piece showed similar deformations compared to the simulated model and this is an indication that the boundary conditions used in the FE model are representative of the physical tee. The fact that the test piece did not fail at an internal pressure above the simulated failure load is mainly attributed to strain hardening of the steel which was not accounted for in the simulated model as elastic perfectly plastic material behaviour was assumed. The pinhole in the acute corner was only through the thickness of the wrapper and did not result in failure of the special under the test pressure, but could lead to crack propogation and sudden failure upon cyclic loading or higher internal pressure. 


\section{Chapter 6}

\section{Conclusions and Future Studies}

\subsection{Conclusions}

The purpose of this project was to determine the accuracy and reliability of reinforcements for steel pipe specials provided by experimental based design models such as AWWA M11. Reinforcements in accordance with this design was described in detail with the objective of providing insight to the need for and importance of reinforcements. The goal was to build representative models that provide designers with some guidelines on the use of numerical tools for the analysis of such reinforcements and provide insight into the efficiency thereof. The numerical model was to reproduce the deformed shape of steel specials subjected to internal pressure and show material stresses and strains with emphasis on high stress regions.

The different types and application of several well-known reinforcements were discussed in detail. Emphasis was placed on typical safety factors for reinforcements in accordance with AWWA M11 and compared to simulated models assuming ductile and elastic perfectly plastic material behaviour. The specials were subjected to hydrostatic pressure loads and boundary conditions chosen accordingly. A comparison was drawn between the failure loads of crotch plate reinforced specials based on the yield strength used by the AWWA M11 model and the higher yield strength of modern steels.

A nonlinear iterative solver, Optistruct, was used to simulate deformations and stresses. The material was assumed to be isotropic with elastic perfectly plastic behaviour. First order bilinear quadrilateral shell elements were chosen for the thin-walled reinforced specials. Attention was given to contact interfaces between pipe shells and intersection edges. Mesh refinement was considered for the intersection region to minimise spurious stress concentrations and it was found that numerical results converged for relatively large elements. This is attributed to the size of specials considered in this study.

The numerical modelling process, assumptions and simulated results were validated with a representative physical test. Tensile tests were performed 
to determine the mechanical properties of the test piece material. It was found that the deformed shape of the physical test compared well with the corresponding simulated model and comparisons can be made for areas with large deformations.

Stress concentrations identified in the simulated models were compared with possible points of failure on the test piece. It was found that a small crack developed in the acute corner of the test piece. Although the crack did not occur as a result of ductile failure it is more likely to propogate due to the high stresses in the acute corner. Strains were measured at a number of points on the test piece and compared with the same points in the corresponding simulated model. It was found that the simulated strains are very sensitive to the selection of elements due to discontinuous strain contours and the difficulty in selecting the actual element corresponding to the strain gauge location.

The simulations showed that the AWWA M11 model does not seem to be conservative for wrapper and collar reinforced specials when assuming elastic perfectly plastic material behaviour and defining the failure load as the point of nonconvergence of the numerical analysis. Past experience with wrapper and collar reinforced specials in accordance with M11 has shown that wrappers and collars are in fact effective and do not tend to fail. Further improvements to collars and wrappers are thus considered to provide little benefit. The M11 crotch plate design model on the other hand seems to be somewhat overly conservative when compared to simulated ductile failure loads in accordance with the assumptions made in this study. This is partly attributed to the fact that the model is based on steel with a low yield strength, typically much lower than what is available on the market today. Further improvements to crotch plate design would therefore make sense.

This study has shown that the design of reinforcements for steel pipe specials could be improved using numerical tools. FE analysis allows for the design of custom tees and provide the designer with more flexibility on the placement and size of reinforcements as well as material selection.

\subsection{Future Studies}

By designing reinforcements purely based on a static load case the design life of the steel special could be compromised due to fatigue. Special consideration therefore needs to be given to the fatigue life of reinforcements where reinforced specials will be subjected to high frequency cyclic pressure conditions. Further studies are recommended to investigate the effect of fatigue on reinforced specials for such conditions. In this study the listed safety factors do not include for fatigue. Steel pipe specials, used in bulk water pipelines, are generally not subjected to high frequency cyclic loadings and therefore it 
is assumed that the designer's chosen safety factor for the static load case is sufficient in dealing with low cycle fatigue over the design life of the special.

Future studies should consider the effect of brittle failure and failure due to welding or other defects. The effect hereof on safety factors should be considered. The true stress-strain behaviour of steel should be used in future studies to allow for strain hardening. 


\section{List of References}

API Specification 5L (2004). Specification for Line Pipe.

Attwater, I., Anderson, J. and Findlay, G. (1994). Three-dimensional finite element analysis of sphere/cylinder intersections under axisymmetric loading. International Journal of Pressure Vessel and Piping, vol. 57, pp. 231-235.

Aurecon (2010). Sishen pipeline, Kumba Iron Ore Sishen Mine.

AWWA M11 (2004). American Water Works Association, M11 Manual, 4th Edition.

Bailey, D. (2014). Using digital image correlation to measure full fiel strain. Available at: http://http://www.asminternational.org/documents/10192/22116322/ amp17210p23.pdf/69654c95-2a2f-4f5d-b150-b3c65def5e6e, [Accessed 20 October 2016].

Cook, R., Malkus, D., Plesha, M. and Witt, R. (2002). Concepts and Applications of Finite Element Analysis, Fourth Edition. Elsevier.

Cook, R. and Young, W. (1999). Advanced Mechanics of Materials, Second Edition. Prentice Hall, New Jersey.

Dekker, C. (1993). Comparison of local load stress calculation methods for nozzles on cylinders. International Journal of Pressure Vessel and Piping, vol. 58, pp. 203-213.

Dekker, C. and Bos, H. (1997). Nozzles-on external loads and internal pressure. International Journal of Pressure Vessel and Piping, vol. 72, pp. 1-18.

Dekker, C. and Brink, H. (2000). Nozzles on spheres with outward weld area under internal pressure analysed by fem and thin shell theory. International Journal of Pressure Vessel and Piping, vol. 77, pp. 399-415.

Dekker, C. and Stikvoort, W. (1997). Pressure stress intensity at nozzles on cylindrical vessels: a comparison of calculation methods. International Journal of Pressure Vessel and Piping, vol. 74, pp. 121-128.

EN13480-3 (2012). Metal Industrial Piping. Part 3. Design and Calculation.

Finlay, J., Rothwell, G., English, R. and Montgomery, R. (2003). Effective stress factors for reinforced butt-welded branch outlets subjected to internal pressure 
or external moment loads. International Journal of Pressure Vessel and Piping, vol. 80 , pp. 311-331.

Inventor (2009). Benefits of using autodesk inventor simulation suite in engineering design. Available at: http://images.autodesk.com/adsk/files/inventor_ simulation_suite_whitepaper-final_8-200.pdf, [Accessed 12 June 2014].

Lekkerkerker, J. (1972). The determination of elastic stresses near cylinder-tocylinder intersections. Nuclear Engineering and Design, vol. 20, pp. 57-84.

McIntyre, H., Ashton, J. and Gill, S. (1977). Limit analysis of a pad reinforced flush nozzle in a spherical pressure vessel. International Journal of Mechanical Sciences, vol. 19, pp. 399-412.

Qadir, M. (2008). Stress and fatigue analysis of pressurised vessel-nozzle intersections with and without local wall thinning.

Quality Tube Services (2016). Sans 719 related fittings. Available at: http: //qualitytube.co.za/2011/05/sabs-719-related-fittings/\#5, [Accessed 30 August 2016].

Roith, B., Alexander, T. and Frank, R. (2007). Integrated finite element analysis (fea) in three dimensional computer aided design programs (cad)-overview and comparison. Available at: http:www.designsociety.org/publication/ 25371/integrated_finite_element_analysis_fea_in_three-dimensional_ computer_aided_design_programs_cad-overview_and_comparison, [Accessed 12 June 2014].

Sang, Z., Xue, L., Lin, Y. and Widera, G. (2002). Limit and burst pressures for a cylindrical shell intersection with intermediate diameter ratio. International Journal of Pressure Vessel and Piping, vol. 79, pp. 341-349.

SANS 719 Standard (2011). Electric welded low carbon steel pipes for aqueous fluids (large bore).

Stephenson, D. (1979). Pipeline Design for Water Engineers, 2nd Edition. Elsevier.

Swanson et al., H. (1955). Design of wye branches for steel pipe. American Water Works Association, vol. 47, no. 6, pp. 581-630.

Visual Analysis (2015). Online user guide. Available at: https://www.iesweb.com/ products/visualanalysis/help/model/plateelements.html, [Accessed $10 \mathrm{Au}-$ gust 2015].

Xu, J., Sun, B. and Koplik, B. (1999). Local pressure stresses on lateral pipe-nozzle with various angles of intersection. Nuclear Engineering and Design, vol. 199, pp. 335-340. 


\section{Appendices}




\section{Appendix A}

\section{Crotch Plate Nomograph}

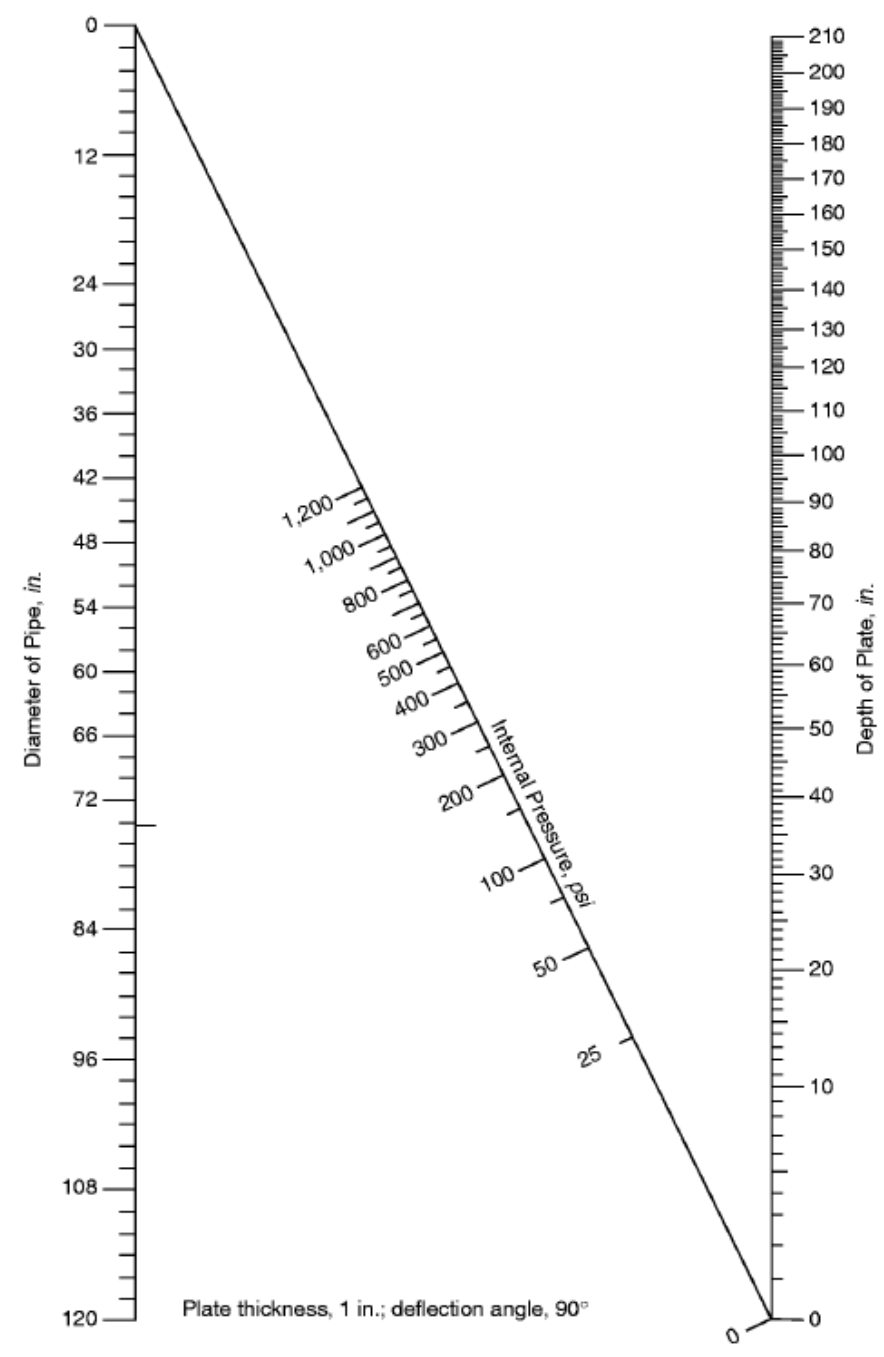

Figure A.1: Nomograph for selecting plate depths in reinforcements (AWWA M11) 


\section{Appendix B}

\section{Pressure vs Strain Curves}

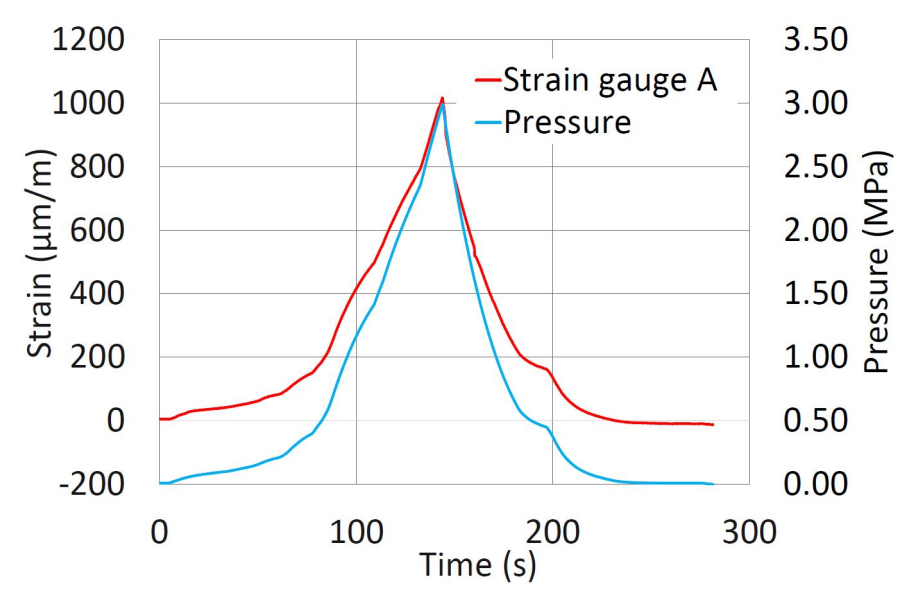

Figure B.1: Pressure vs strain - strain gauge A.

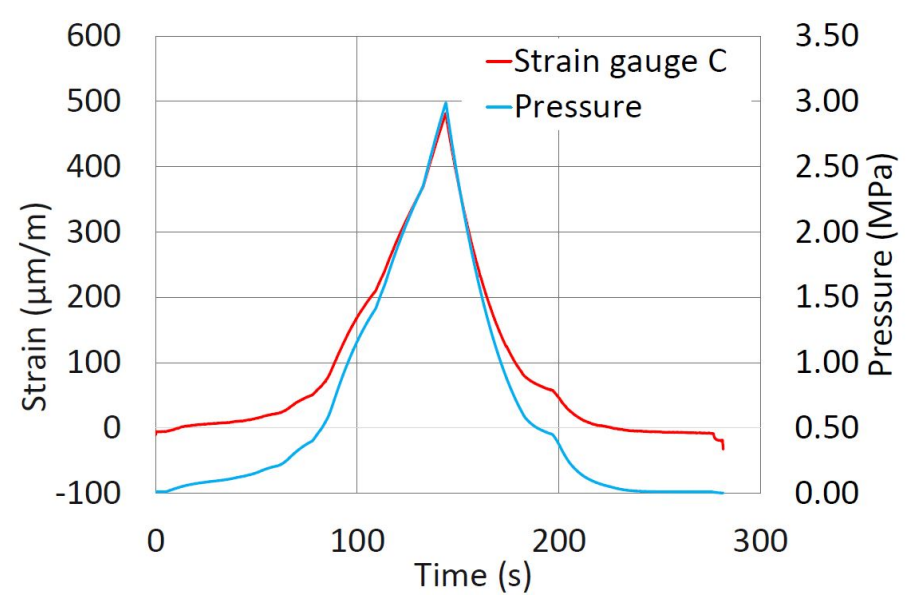

Figure B.2: Pressure vs strain - strain gauge C. 


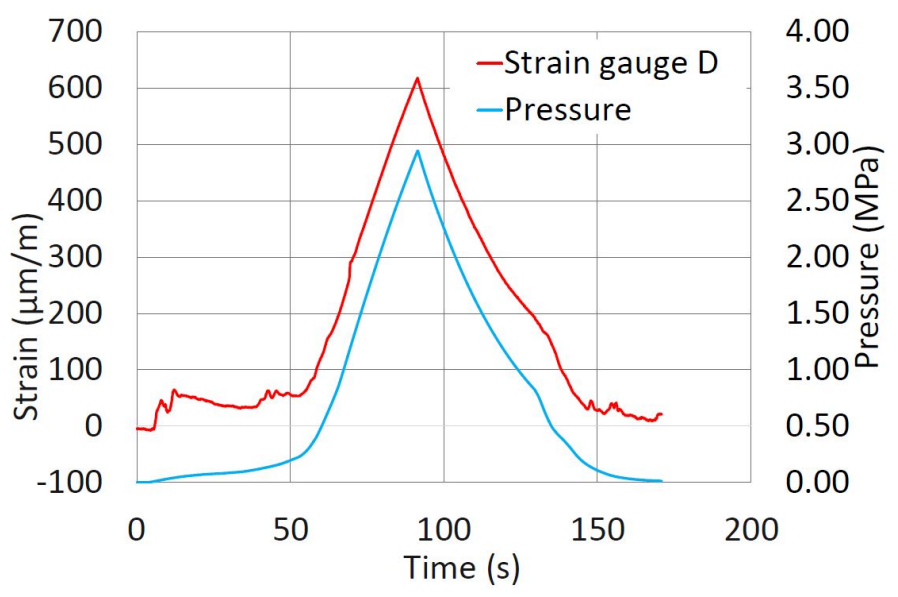

Figure B.3: Pressure vs strain - strain gauge D.

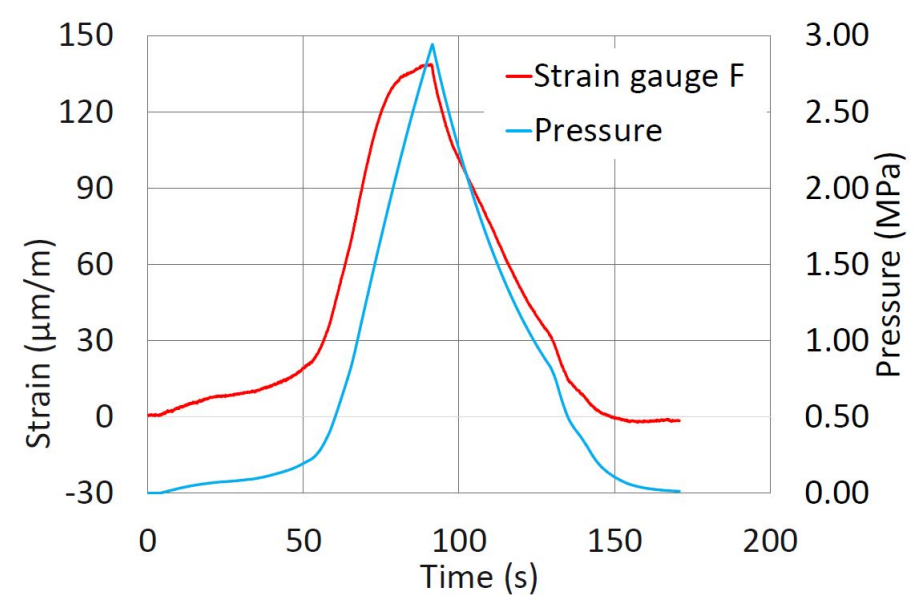

Figure B.4: Pressure vs strain - strain gauge F.

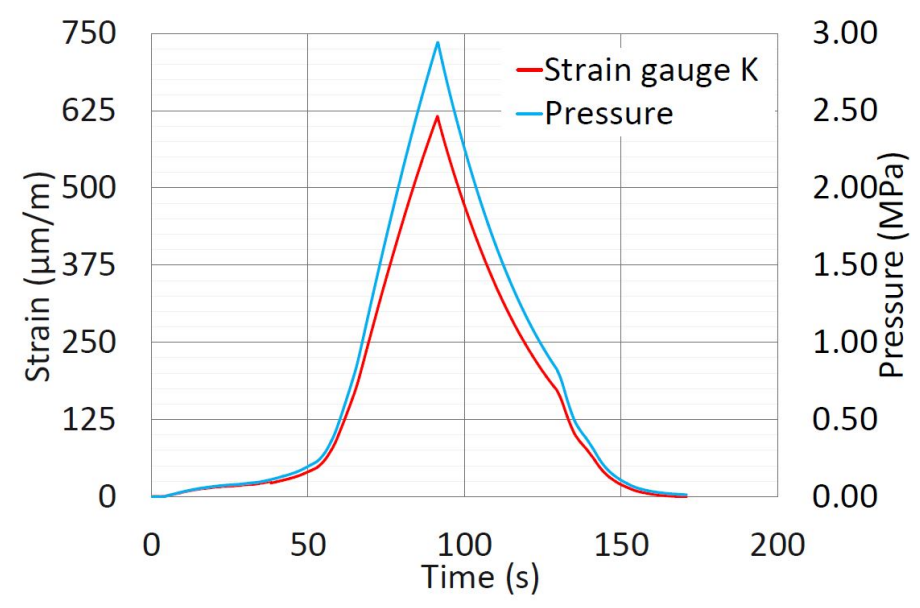

Figure B.5: Pressure vs strain - strain gauge K. 


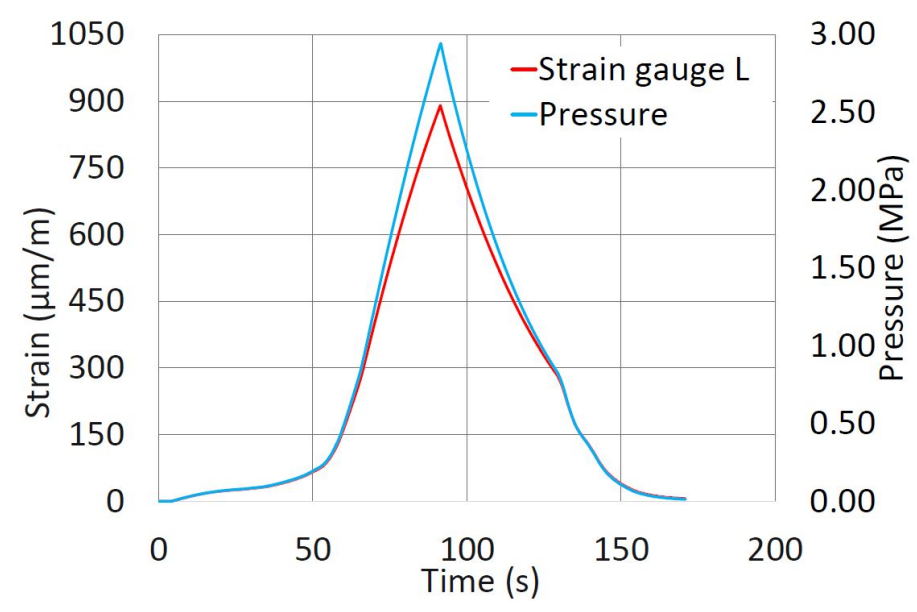

Figure B.6: Pressure vs strain - strain gauge L.

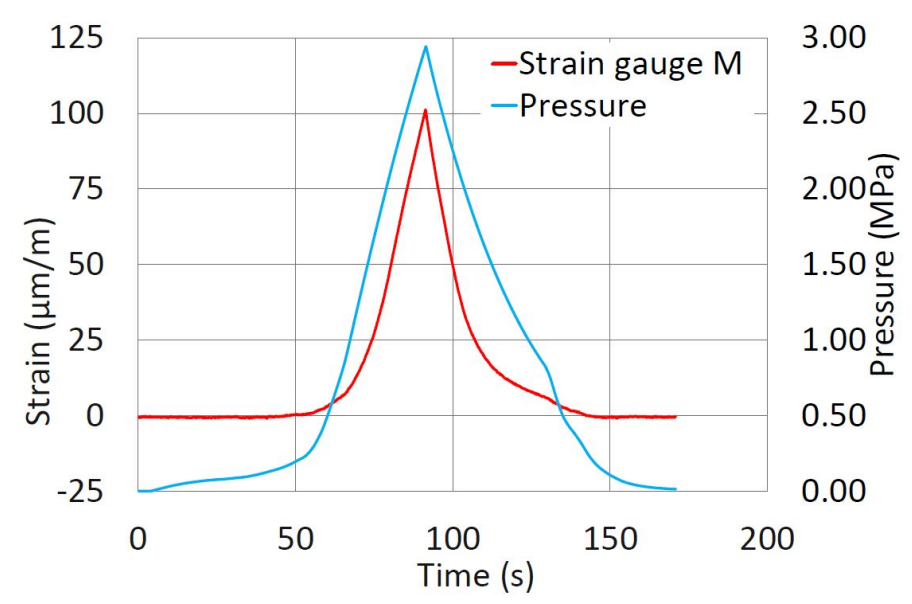

Figure B.7: Pressure vs strain - strain gauge M.

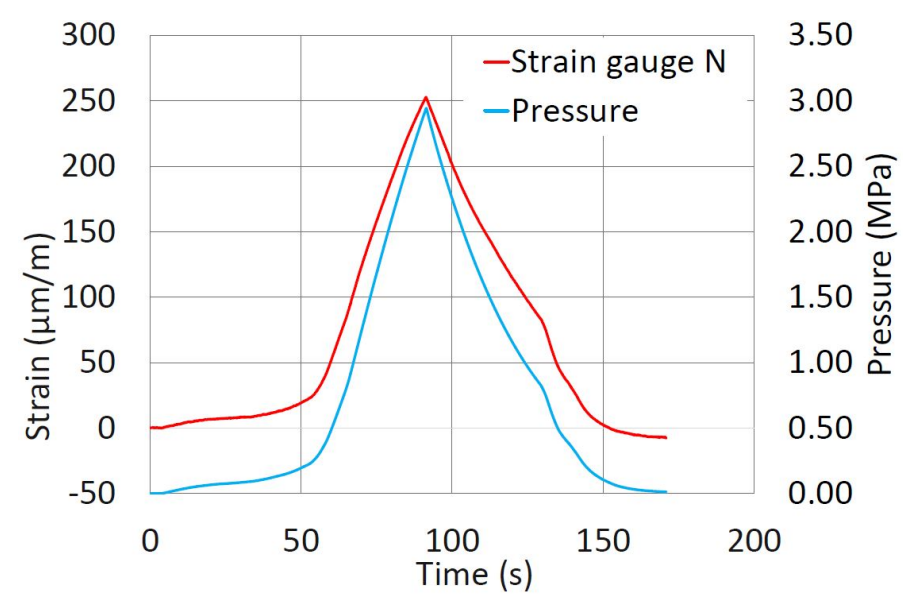

Figure B.8: Pressure vs strain - strain gauge N. 


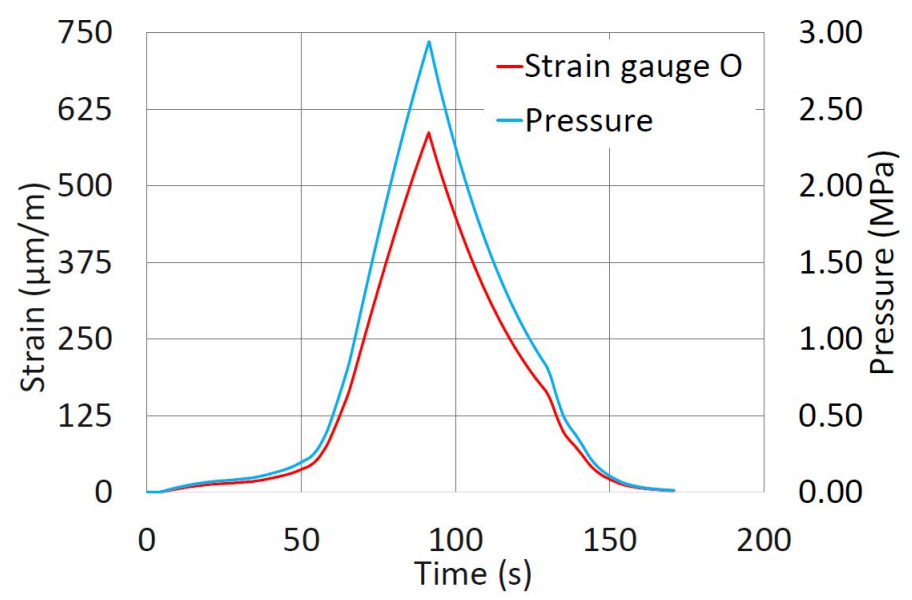

Figure B.9: Pressure vs strain - strain gauge O.

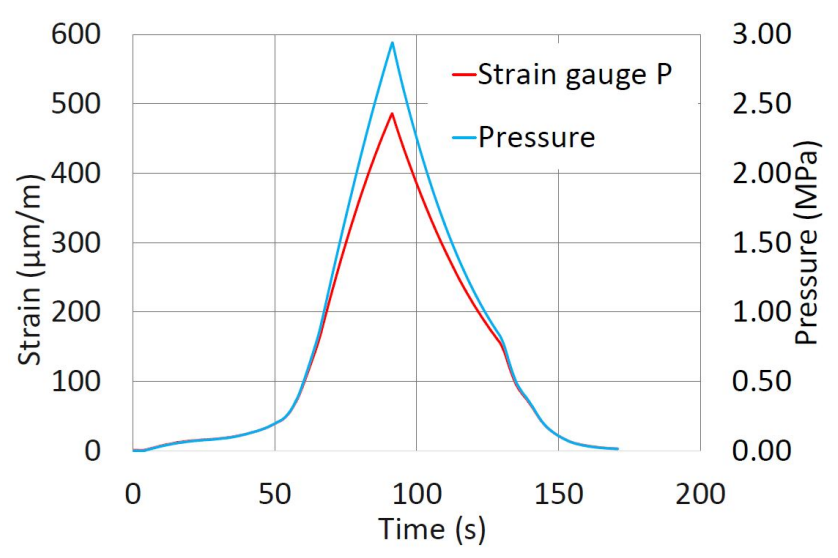

Figure B.10: Pressure vs strain - strain gauge P.

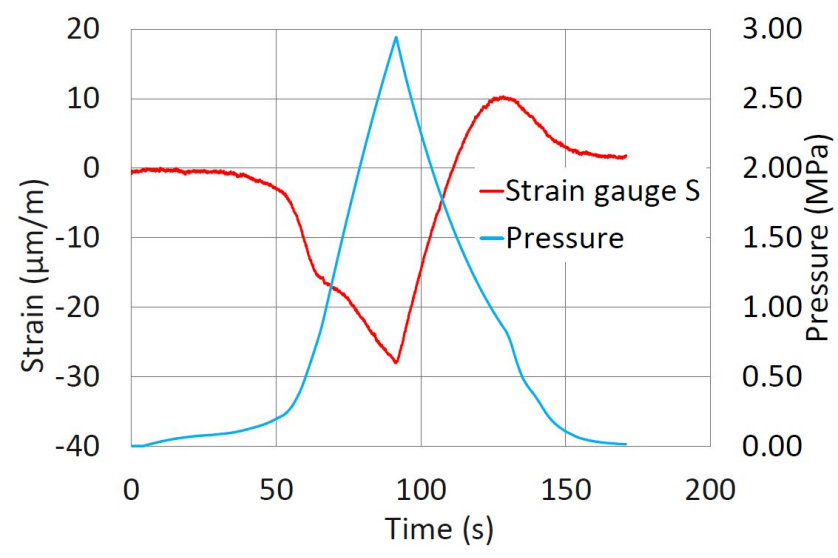

Figure B.11: Pressure vs strain - strain gauge S. 


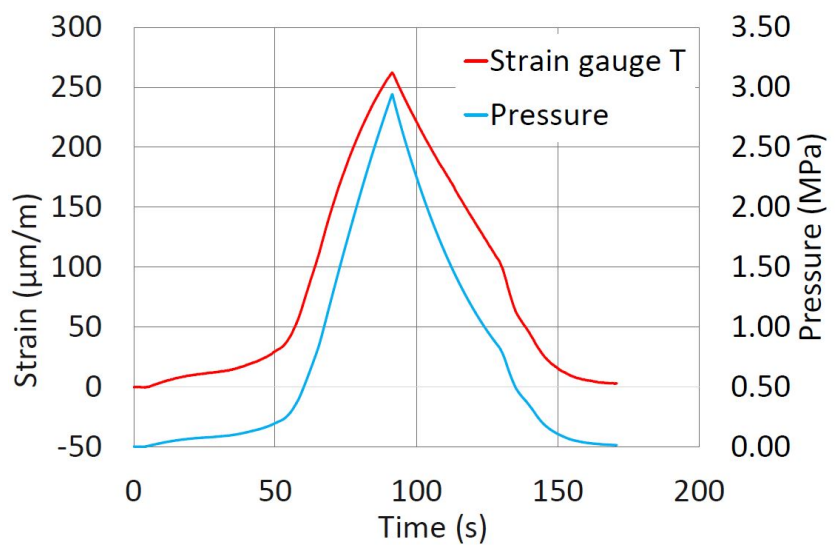

Figure B.12: Pressure vs strain - strain gauge T.

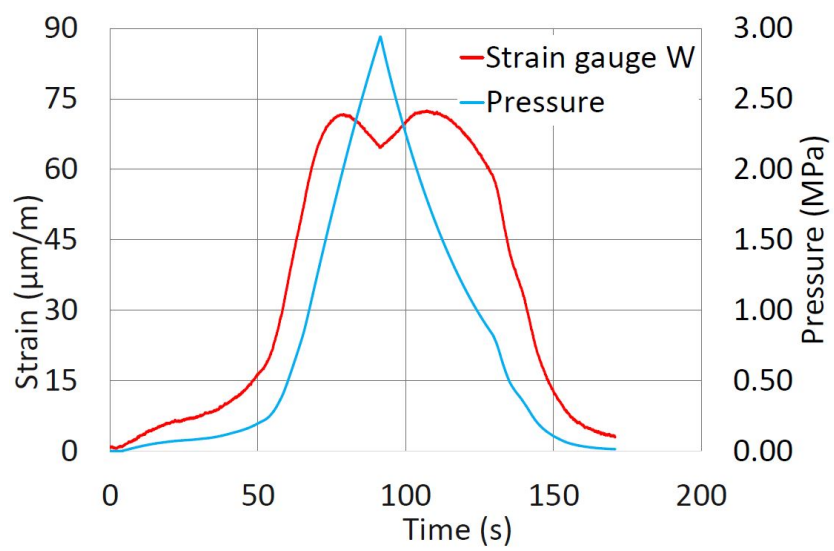

Figure B.13: Pressure vs strain - strain gauge W.

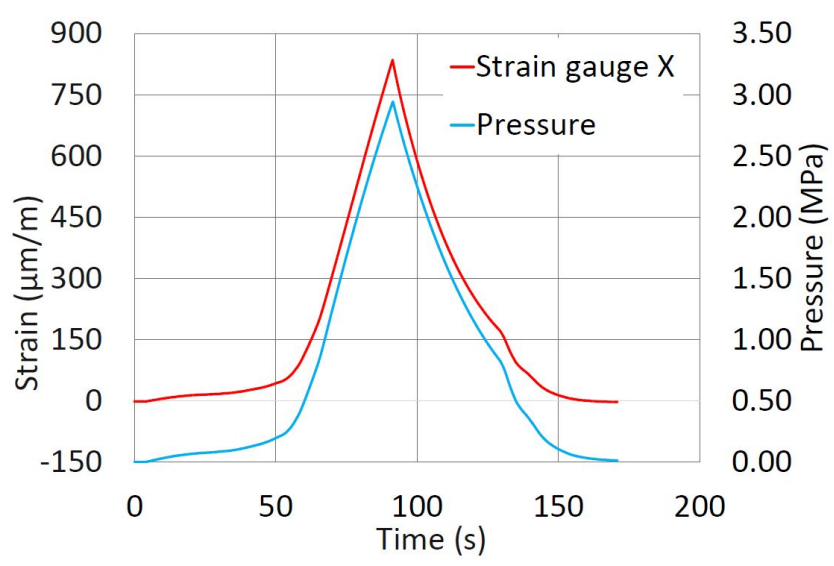

Figure B.14: Pressure vs strain - strain gauge X. 